\section{Tracy Haack ${ }^{*}$}

and

Hampton N. Shirer

Department of Meteorology

The Pennsylvania State University

University Park, PA 16802

*Present affiliation:

Naval Oceanographic and Atmospheric

Research Laboratory,

Monterey, CA 93943-5006

July 1991

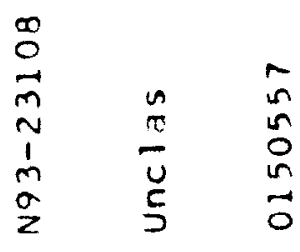

$\stackrel{\sim}{0}$

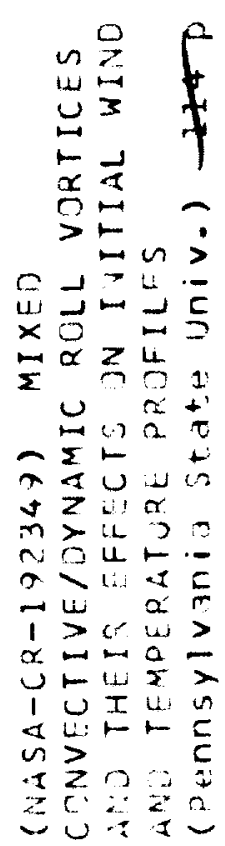




\begin{abstract}
The onset and development of both dynamically and convectively forced boundary layer rolls are studied with linear and nonlinear analyses of a truncated spectral model of shallow Boussinesq flow. Emphasis is given here on the energetics of the dominant roll modes, on the magnitudes of the roll-induced modifications of the initial basic state wind and temperature profiles, and on the sensitivity of the linear stability results to the use of modified profiles as basic states. It is demonstrated that the roll circulations can produce substantial changes to the cross-roll component of the initial wind profile and that significant changes in orientation angle estimates can result from use of a roll-modified profile in the stability analysis. These results demonstrate that roll contributions must be removed from observed background wind profiles before using them to investigate the mechanisms underlying actual secondary flows in the boundary layer.

The model is developed quite generally to accept arbitrary basic state wind profiles as dynamic forcing. An Ekman profile is chosen here merely to provide a means for easy comparison with other theoretical boundary layer studies; the ultimate application of the model is to study observed boundary layer profiles. Results of the analytic stability analysis are validated by comparing them with results from a larger linear model. For an appropriate Ekman depth, a complete set of transition curves is given in forcing parameter space for roll modes driven both thermally and dynamically. Preferred orientation angles, horizontal wavelengths and propagation frequencies, as well as energetics and wind profile modifications, are all shown to agree rather well with results from studies on Ekman layers as well as with studies on near-neutral and convective atmospheric boundary layers.
\end{abstract}




\section{Introduction}

Observational evidence of stable secondary flows in the planetary boundary layer is abundant. Early scientists (Langmuir 1938) noted that long rows of seaweed floated parallel to the wind, and Woodcock (1942) observed that the soaring patterns of seagulls correlated with convective updrafts. More recently, satellite pictures have shown lines of sand dunes in the Sahara aligned with the prevailing winds and numerous examples of cloud streets during cold air outbreaks over water (Brown 1980). Atmospheric roll circulations consistently occur in boundary layers having a slightly unstable stratification and moderately strong winds. Typical wavelengths for these rolls range from two to eight $\mathrm{km}$ and are about three times the circulation depth; roll orientations are approximately $15^{\circ}$ to the left of the geostrophic flow; phase speeds average one to two $\mathrm{m} / \mathrm{s}$; and propagation periods may be from $15 \mathrm{~min}$ to $2 \mathrm{hrs}$ (Brown 1972; LeMone 1973).

As reviewed extensively elsewhere (Brown 1980; Stensrud 1987a), roll circulations are driven by both convective and dynamic instability mechanisms. Convective instability produces Rayleigh/Bénard circulations when a vertical potential temperature gradient exists at the surface. For wind speeds greater than a few meters per second, these thermal cells align in linear cloud bands that are nearly parallel to the direction of mean wind shear (Kuettner 1959, 1971). Studies of neutral atmospheres (Lilly 1966; Faller and Kaylor 1967) indicate that two dynamic mechanisms can also induce secondary flows. The inflection point instability mechanism generates roll circulations when sufficient energy is extracted from the shear in the roll-perpendicular wind component. In contrast, rolls excited by the parallel instability mechanism require Coriolis turning to extract energy from the roll-parallel wind component; typically, the Coriolis conversion terms are much smaller in magnitude than are the other energy contributions, and so the parallel instability mechanism is believed to be of lesser importance in the atmosphere (Brown 1972; LeMone 1973, Brümmer 1985; Chlond 
1987). Brown (1980) notes that the convective and inflection point instability mechanisms appear to be sufficient to explain most geophysical lineal flow patterns.

Each of these mechanisms has been studied extensively using a variety of theoretical approaches. Lilly (1966) and Brown (1970) investigated the dynamic mechanisms using linear models based on the partial differential equations for the neutral Ekman boundary layer. Etling (1971), Brown (1972), and Asai and Nakasuj (1973) are among those who utilized linear partial differential models to study the mixed convective/dynamic instabilities of an Ekman layer, while others, including Kuo (1963), Asai (1970a, 1970b, 1972), Kuettner (1971), Sun (1978), and Shirer (1980) considered the linear boundary layer responses to other sheared flows. In addition, high resolution nonlinear models have been developed for numerical study of both the Ekman layer (e.g. Faller and Kaylor 1966, 1967) and more general boundary layers (e.g. Faller and Kaylor 1969; Sommeria and LeMone 1978; Mason and Sykes 1980, 1982; Becker 1987; Chlond 1987; Etling and Raasch 1987). Results from the studies mentioned above have provided a basic understanding of:

(i) the instability mechanisms responsible for roll development,

(ii) the preferred roll characteristics, i.e. orientation angles and horizontal wavelengths associated with each mechanism,

(iii) the resulting secondary flow patterns, and

(iv) the profiles of the energetics terms, vertical transports and modifications to the basic state.

A host of studies that incorporate measurements of observed boundary layer circulations (e.g. Kuettner 1971; LeMone 1973; LeMone and Pennell 1976; Sommeria and LeMone 1978; Weston 1980; Kelly 1984; Walter and Overland 1984; Becker 1987; Chlond 1987; Etling and Raasch 1987) have in general confirmed these theoretical results.

Although the above studies have revealed a great deal about the fundamental properties of boundary layer rolls, they have provided only snapshots of the expected behavior in the 
forcing and response parameter spaces. In particular, large, high-resolution models can not provide a very complete picture because they can be integrated only for a limited number of parameter values; moreover, in many cases the modeled rolls are not able to respond by changing their orientation angle because usually this angle must be specified in advance. In contrast, most linear models provide the response parameters as output, but they typically are developed for study of only one, usually the Ekman, profile and so their linear stability results can not be generalized easily to that expected for an atmospheric profile. Hence, a simple model capable of accepting arbitrary profiles is required for investigation of observed boundary layer flows. Existing attempts at comparing observations with model results tend to use the observed, roll-modified wind profiles as input rather than the (probably unobserved) pre-roll state whose instability actually led to the rolls themselves. The sensitivity of the linear analysis to use of such incorrect profiles must be investigated further; if significant sensitivity is found, then a simple means for estimating the probable roll modification is needed. Therefore, a modeling approach must be used that allows representation of the roll modes with a model large enough to capture the important dynamic and thermodynamic modes but still small enough to study analytically.

Such a modeling approach is codified in the low-order spectral technique pioneered by Lorenz (1963) and discussed extensively in the book edited by Shirer (1987). In these nonlinear models, the dependent variables are represented by truncated Fourier expansions composed of temporally dependent amplitude coefficients and spatially dependent trigonometric basis functions. For boundary layer roll studies, the spatial characteristics are often given by a single horizontal harmonic and one (Shirer 1980, 1986) or two (Stensrud and Shirer 1988) vertical harmonics. The possibility of successfully using such severe truncations is suggested, for example, by ground observations (Kuettner 1959, 1971), examination of satellite images (Brown 1980), time-height cross sections of tower data (LeMone 1973), and cross sections given by aircraft data (LeMone and Pennell 1976; Brümmer 1985). Basing the 
truncated models on the complete nonlinear Boussinesq equations allows study of both single and mixed instability mechanisms over a wide range of environmental wind shears and static stabilities (Shirer 1986). The onset of a roll mode is represented by a bifurcation from a motionless conductive state, and each critical forcing parameter value is given by a root of an analytically derived polynomial equation (Stensrud 1987a). Consideration of a large range of parameter space can therefore be performed quickly and efficiently. Moreover, because the basic state in these models is represented by a truncated Fourier series (Stensrud and Shirer 1988), the spectral modeling approach is ideal for direct comparisons between model results and observations, as was done by Shirer and Brümmer (1986) and Stensrud and Shirer (1988). Although reasonable agreement was obtained between their results and observations taken during the 1981 KonTur experiment (Brümmer 1985), the models were somewhat limited because the potential roll modification of the initial background wind could not be considered.

In order to investigate the possible modification of the initial basic state by boundary layer rolls, we develop in sections 2,3 and 4 a new nonlinear 14-coefficient spectral model of two-dimensional shallow Boussinesq flow that is forced both convectively and dynamically. In section 5 we qualitatively compare our results with those of previous theoretical and observational studies, and in section 6 we investigate the sensitivity of the stability results to the use of roll-modified profiles. In our study, we use an Ekman profile because it has been used in the vast majority of previous theoretical studies; however, the ultimate application of the model is to observed atmospheric profiles. 


\section{Model development}

To model boundary layer roll circulations, we use the shallow Boussinesq equations (Dutton and Ficht1 1969). Cloud streets typically form in the upward branches of these circulations, indicating the presence of two-dimensional roll patterns. However, we may neglect latent heating effects by assuming that the cloud area is small (Laufersweiler and Shirer 1989). The rolls are represented by perturbations superimposed on a timeindependent, hydrostatic and horizontally moving basic state (Shirer 1980, 1986). Here, the initial cross-roll wind profile $U(z)$ is approximated by a truncated Fourier series involving two vertical wavenumbers $q$ and $n$ :

$$
\begin{aligned}
\mathrm{U}(\mathrm{z})=\mathrm{U}_{0}+\mathrm{U}_{1} \sin (\mathrm{q} & \left.\pi \mathrm{z} / \mathrm{z}_{\mathrm{T}}\right)+\mathrm{U}_{2} \cos \left(\mathrm{q} \pi \mathrm{z} / \mathrm{z}_{\mathrm{T}}\right) \\
& +\mathrm{U}_{3} \sin \left(\mathrm{n} \pi \mathrm{z} / \mathrm{z}_{\mathrm{T}}\right)+\mathrm{U}_{4} \cos \left(\mathrm{n} \pi \mathrm{z} / \mathrm{z}_{\mathrm{T}}\right)
\end{aligned}
$$

where $z_{T}$ specifies the height of the domain. The coefficients $U_{i}$ are found from appropriate Fourier integrals, as described in section 5 . As a first-order approximation, the initial temperature profile $T_{0}(z)$ varies linearly with height and is defined in two parts:

$$
\mathrm{T}_{0}(\mathrm{z})=\mathrm{T}_{\mathrm{l}}+\mathrm{T}_{\mathrm{f}}=\left(\mathrm{T}_{\mathrm{sa}}-\gamma_{\mathrm{e}} \mathrm{z}\right)+\left(\mathrm{T}_{\mathrm{lb}}-\mathrm{T}_{\mathrm{sa}}\right)\left(\mathrm{z}_{\mathrm{T}}-\mathrm{z}\right) / \mathrm{z}_{\mathrm{T}}
$$

The temperature $T_{1}$ represents the contribution owing to the environmental lapse rate $\gamma_{e}$ and the temperature $T_{f}$ is a vertically distributed surface forcing contribution based upon the difference between the lower boundary temperature $T_{1 b}$ and the surface air temperature $T_{s a}$.

We assume that the perturbations possess a two-dimensional structure and so neglect all roll-parallel variations. Thus, the horizontal and vertical equations of motion may be combined into a single vorticity equation using the following form for the stream function: $\partial \psi / \partial z=-u^{\prime}$ and $\partial \psi / \partial x=w^{\prime}$. We also make the standard assumption (e.g. Brown 1970) that the Coriolis terms are small in magnitude and so do not contribute significantly to roll 
development. Although Brown (1970) proposed a profile-modification mechanism that depended on the Coriolis parameter in his mean wind equations, we investigate here whether a more rapid adjustment mechanism is possible that depends only on the nonlinear coupling terms. Evidence for relatively rapid changes to observed cloud streets, implying rapid changes to the background profiles, is given by Brümmer (1985). The above simplifications are strictly valid provided that the perturbations reach a steady state within a short time scale, two to four hours, and that only moderate supercritical forcing rates are considered in the temporal integrations.

The horizontal domain is infinite and cyclically continuous at $x=0$ and $x=L$, where $L$ is the roll wavelength. Vertically the domain ranges from $z=0$ to $z=z_{T}$. For simplicity these boundaries are assumed to be rigid, stress-free and perfectly conducting. In dimensionless variables denoted by an asterisk, we have a domain defined by $0 \leq x^{*} \leq 2 \pi$ and $0 \leq z^{*} \leq \pi$.

Using the above assumptions, we derive a partial differential system containing equations for the perturbation stream function $\psi$ and perturbation temperature T'. We write the dimensionless equations representing boundary layer flow as (Stensrud 1987a; Haack-Hirschberg 1988)

$$
\begin{gathered}
\frac{\partial}{\partial \mathrm{t} *}\left(\bar{\nabla} \psi^{*}\right)+\mathrm{K}^{*}\left(\psi^{*}, \bar{\nabla} 2 \psi^{*}\right)-\mathrm{P} \frac{\partial \mathrm{T}^{*}}{\partial \mathrm{x}^{*}}+\mathrm{P} \operatorname{Re} \mathrm{U}^{*} \frac{\partial}{\partial \mathrm{x}^{*}}\left(\tilde{\nabla}^{2} \psi^{*}\right) \\
-\mathrm{P} \operatorname{Re} \frac{\partial \psi^{*}}{\partial \mathrm{x}^{*}} \frac{\partial^{2} \mathrm{U}^{*}}{\partial \mathrm{L}^{*}}-\frac{\mathrm{P}}{\mathrm{a}} \tilde{\nabla} \psi^{*}=0 \\
\frac{\partial \mathrm{T}^{*}}{\partial \mathrm{t}}+\mathrm{K}^{*}\left(\psi^{*}, \mathrm{~T}^{*}\right)-\operatorname{Ra} \frac{\partial \psi^{*}}{\partial \mathrm{x}^{*}}+\mathrm{P} \operatorname{Re} \mathrm{U}^{*} \frac{\partial \mathrm{T}^{*}}{\partial \mathrm{x}^{*}} \\
-\frac{1}{\mathrm{a}} \tilde{\nabla} \mathrm{T}^{*}=0
\end{gathered}
$$

where the tilde denotes a dimensionless Laplacian operator, and $\mathrm{K}^{*}$ denotes a dimensionless Jacobian operator. 
The dimensionless forms lead to two forcing parameters in $(2.3)-(2.4)$. The Reynolds number Re is given by

$$
\operatorname{Re}=\left|\underset{\sim}{V}\left(\mathrm{z}_{\mathrm{T}}\right)\right| \mathrm{z}_{\mathrm{T}} / \pi \nu
$$

and represents dynamic forcing imparted by the basic wind. The Rayleigh number $\mathrm{Ra}$ is given by

$$
R a=R a_{l}+R a_{f}=\left[\left(\gamma_{e}-\gamma_{d}\right) z_{T}+\left(T_{l b}-T_{s a}\right)\right] g z_{T} T^{3} / \pi^{4} T_{s a} \nu \kappa
$$

and represents thermodynamic forcing. Here, we have separated the thermal forcing into two terms in order to define the energetics (section 3) and to aid eventual application of the model to the atmosphere: $\mathrm{Ra}_{1}<0$ is the slightly stable boundary layer contribution that is proportional to the positive potential temperature gradient or equivalently to the difference $\gamma_{d}-\gamma_{e}$ between the dry and environmental adiabatic lapse rates, and $\mathrm{Ra}_{\mathrm{f}}$ is the thermal forcing contribution that is proportional to the difference $T_{1 b}-T_{s a}$ between the lower boundary and surface air temperatures.

Three other dimensionless variables appear in the system (2.3) - (2.4). The eddy Prandtl number $\mathrm{P}=\nu / \kappa$ is the ratio of the constant eddy viscosity $\nu$ and the constant eddy thermometric conductivity $\kappa$; as noted by Laufersweiler and Shirer (1989), this assumption is reasonable for modeling boundary layer rolls. Although difficult to estimate, we may use the results of Brümmer (1985) to guide our choice of a value for $v$; normally atmospheric values of $P$ are assumed to be near 1 . The roll aspect ratio $a$ is defined as

$$
a=\frac{2 z_{T}}{L}
$$

in which the domain height $z_{T}$ is generally chosen to be the cloud top or inversion base, and the roll wavelength $\mathrm{L}$ is obtained from the value of $a$. The variable $U^{*}\left(z^{*}\right)$ represents the dimensionless cross-roll wind profile. Owing to the dimensionless forms chosen, we have the 
constraint $\left|\mathrm{U}^{*}(\pi)\right|=1$. In this system, the roll-parallel wind component $\mathrm{V}^{*}\left(\mathrm{z}^{*}\right)$ has been decoupled via the elimination of the Coriolis parameter and the assumption of twodimensionality. Thus we are not considering some longitudinal contributions that may be important.

Using the formula,

$$
\mathrm{U}(\mathrm{z})=\mathrm{U}_{\mathrm{s}}(\mathrm{z}) \sin (\beta)-\mathrm{V}_{\mathrm{s}}(\mathrm{z}) \cos (\beta)
$$

we may rotate the cross-roll winds into a standard coordinate system for which the eastward $\mathrm{U}_{\mathrm{s}}(\mathrm{z})$ and northward $\mathrm{V}_{\mathrm{s}}(\mathrm{z})$ wind components are labeled with the subscript $\mathrm{s}$ (Fig. 1). Here $\beta$ is the roll orientation angle that is defined to be the angle between a standard reference direction $\mathrm{x}_{\mathbf{s}}$ and the roll axis $\mathrm{y}$; thus, for example, when $\beta=0^{\circ}, \mathrm{U}=-\mathrm{V}_{\mathbf{s}}$. Positive values are measured counterclockwise, negative values clockwise. For a given wind profile, the values of a and $\beta$ represent geometric characteristics of the modeled roll circulations. Because the cross-roll wind $\mathrm{U}(\mathrm{z})$ changes as the value of $\beta$ is changed, the two-dimensional solutions can react to the complete horizontal wind profile by choosing an optimal angle $\beta_{\mathrm{p}}$. In this way we are able to incorporate some aspects of both horizontal dimensions into the modeled dynamic forcing.

Fourier expansions, composed of temporally dependent amplitudes and spatially dependent trigonometric functions, are used to represent the dependent variables $\psi^{*}$ and $\mathrm{T}^{*}$ :

$$
\begin{aligned}
& \psi^{*}\left(\mathrm{x}^{*}, \mathrm{z}^{*}, \mathrm{t}^{*}\right)=\psi_{\mathrm{r}}^{*}\left(\mathrm{x}^{*}, \mathrm{z}^{*}, \mathrm{t}^{*}\right)+\psi_{b}^{*}\left(\mathrm{z}^{*}, \mathrm{t}^{*}\right) \\
& =\left[\psi_{1}(\mathrm{t} *) \sin \left(\mathrm{x}^{*}\right) \sin \left(\mathrm{qz} z^{*}\right)+\psi_{2}\left(\mathrm{t}^{*}\right) \cos \left(\mathrm{x}^{*}\right) \sin \left(\mathrm{qz} z^{*}\right)\right. \\
& \left.+\psi_{3}\left(\mathrm{t}^{*}\right) \sin \left(\mathrm{x}^{*}\right) \sin \left(\mathbf{n} \mathbf{z}^{*}\right)+\psi_{4}\left(\mathrm{t}^{*}\right) \cos \left(\mathbf{x}^{*}\right) \sin \left(\mathbf{n} \mathbf{z}^{*}\right)\right] \\
& +\left[\psi_{5}\left(t^{*}\right) \sin \left((\mathrm{q}-\mathrm{n}) \mathbf{z}^{*}\right)+\psi_{6}\left(\mathrm{t}^{*}\right) \sin \left((\mathrm{q}+\mathrm{n}) \mathbf{z}^{*}\right)\right]
\end{aligned}
$$




$$
\begin{aligned}
& \mathrm{T}^{*}\left(\mathrm{x}^{*}, \mathrm{z}^{*}, \mathrm{t}^{*}\right)=\mathrm{T}_{\mathrm{r}}^{*}\left(\mathrm{x}^{*}, \mathrm{z}^{*}, \mathrm{t}^{*}\right)+\mathrm{T}_{\mathrm{b}}^{*}\left(\mathrm{z}^{*}, \mathrm{t}^{*}\right) \\
& =\left[\mathrm{T}_{1}\left(\mathrm{t}^{*}\right) \cos \left(\mathrm{x}^{*}\right) \sin \left(\mathrm{qz} \mathrm{z}^{*}\right)+\mathrm{T}_{2}\left(\mathrm{t}^{*}\right) \sin \left(\mathrm{x}^{*}\right) \sin \left(\mathrm{q} \mathrm{z}^{*}\right)\right. \\
& \left.+\mathrm{T}_{3}(\mathrm{t} *) \cos \left(\mathrm{x}^{*}\right) \sin \left(\mathrm{n \mathbf {z } ^ { * }}\right)+\mathrm{T}_{4}(\mathrm{t} *) \sin \left(\mathrm{x}^{*}\right) \sin \left(n \mathbf{z}^{*}\right)\right] \\
& +\left[\mathrm{T}_{5}(\mathrm{t} *) \sin \left((\mathrm{q}-\mathrm{n}) \mathrm{z}^{*}\right)+\mathrm{T}_{6}(\mathrm{t} *) \sin \left((\mathrm{q}+\mathrm{n}) \mathbf{z}^{*}\right)\right. \\
& \left.+\mathrm{T}_{7}\left(\mathrm{t}^{*}\right) \sin \left(2 \mathrm{qz} \mathrm{z}^{*}\right)+\mathrm{T}_{8}\left(\mathrm{t}^{*}\right) \sin \left(2 \mathrm{nz} \mathrm{z}^{*}\right)\right]
\end{aligned}
$$

The roll solutions $\psi_{\mathrm{S}}^{*}$ and $\mathrm{T}_{\mathrm{r}}^{*}$ are given by the eight terms involving spectral components $\psi_{1}$ through $\psi_{4}$ and $\mathrm{T}_{1}$ through $\mathrm{T}_{4}$, while the nonlinear modifications $\psi_{\mathrm{b}}^{*}$ and $\mathrm{T}_{\mathrm{b}}^{*}$ of the initial wind and temperature profiles are given by the six horizontally constant terms involving components $\psi_{5}, \psi_{6}$ and $\mathrm{T}_{5}$ through $\mathrm{T}_{8}$. To permit horizontal roll propagation, both sine and cosine functions of $x^{*}$ are required (Pyle 1987), while to satisfy the vertical boundary conditions, only sine functions of $z^{*}$ are used. Two general wavenumbers, $q$ and $n$, are included in the vertical representation of $(2.9)-(2.10)$ for study of the inflection point instability and for improved representation of the thermal instability, which requires at least one harmonic (Stensrud 1987a). Although the above truncations are sufficient for the approximation of most simple flow patterns, more spectral modes would be needed to quantify completely the initial profile $U(z)$ and the solutions at larger supercritical values of the forcing. In section 5 , we verify that the Ekman profile we study produces stability results that are not sensitive to increased vertical resolution in the model.

Upon substituting the expansions for $\psi^{*}$ and $\mathrm{T}^{*}$ into $(2.3)-(2.4)$ and integrating appropriately over the domain, we obtain the 14 time-dependent spectral equations given in Appendix A. We note that the nonlinear terms in the spectral equations $(A 5)-(A 6)$ and (A11) - (A14) for the profile modification coefficients correspond to the usual relations $\partial U^{*} / \partial t^{*}=-\partial \overline{u^{*} w^{*}} / \partial z^{*}$ and $\partial T_{0}^{*} / \partial t^{*}=-\partial \overline{w^{*} T^{*}} / \partial z^{*}$, where the overbars denote horizontal averages (e.g. Chlond 1987). Definitions for each of the coefficients $a_{i}, b_{i}, c_{i}$ and $d_{i}$ in these equations are shown in Table A1. Coefficients $c_{i}$, multiplying the dynamic forcing parameter 
Re, contain the Fourier coefficients $\Lambda_{\mathfrak{i}}$ and $\Gamma_{\mathfrak{i}}$ of the dimensionless cross-roll wind profile $\mathrm{U}^{*}\left(\mathbf{z}^{*}\right)$. The Fourier integrals are defined in Table A2. Values for $\Lambda_{\mathrm{i}}$ and $\Gamma_{\mathrm{i}}$ may be obtained from either idealized or observed wind profiles. Here, idealized results using the Ekman wind profile are analyzed, while in a companion paper, observational results from the 1987 stratocumulus experiment FIRE are considered; a preliminary report of these results is given in Shirer and Haack (1990).

\section{Energetics}

The dimensionless Boussinesq system (2.3) - (2.4) contains sources and sinks of both available potential $\mathrm{AE}$ and kinetic $\mathrm{KE}$ energies that contribute to the growth and development of roll circulations. Sources of roll energy are of both thermal and dynamic type, while sinks inciude eddy dissipation and roll modifications of the initial background profiles. These individual energy contributions and the interactions between the secondary and background flow may be analyzed by separating the dimensionless energetics components into four parts: roll kinetic KER and available potential AER energies, and background kinetic KEB and available potential AEB energies. Fundamentally, the available energy definitions change with the sign of $R a=R a_{1}+R a_{f}$ in (2.6).

A common environment for secondary instability is produced when cold air overspreads warmer water. These conditions create strong capping temperature inversions for which a constant domain height $z_{T}$ is appropriate. Typically in this case, the boundary layer has a slightly stable initial lapse rate $\left(\operatorname{Ra}_{1}<0\right)$, and buoyant forcing at the sea surface $\left(\operatorname{Ra}_{f}>0\right)$. When $\left|R a_{f}\right|>\left|R a_{1}\right|$, the value of the total thermal forcing rate $R a$ is positive, and contributions from both thermal and dynamic sources can force secondary instabilities; conversely, when $\left|R a_{f}\right|<\left|R a_{1}\right|$, the value of $R a$ is negative, and only dynamic sources can force the instabilities. 
Here we examine the case $\mathrm{Ra}>0$ for which both roll thermal generation and AER/KER conversion, or heat flux, terms are obtained. Although similar formulations of the energetics have appeared elsewhere (e.g. Kaylor and Faller 1972; LeMone 1973), we include our version here to make clear the sources and sinks of energy in the present model, with particular attention given to the origins of the profile modification terms. The appropriate definitions for $\mathrm{KER}, \mathrm{AER}, \mathrm{KEB}$ and $\mathrm{AEB}$ are

$$
\begin{aligned}
& \mathrm{KER}=\frac{1}{2} \int_{0}^{2 \pi} \int_{0}^{\pi}\left|\bar{\nabla} \psi_{\mathrm{s}}^{*}\right|^{2} \mathrm{~d} \mathrm{x}^{*} \mathrm{~d} \mathrm{z}^{*} \\
& \mathrm{AER}=\frac{1}{\left(-\mathrm{R} \mathrm{a}_{1}\right)}\left[\frac{1}{2} \int_{0}^{2 \pi} \int_{0}^{\pi} \mathrm{P} \mathrm{T}_{\mathrm{r}}^{*} \mathrm{dx}^{*} \mathrm{dz} \mathrm{z}^{*}\right] \\
& \mathrm{KEB}=\frac{1}{2} \int_{0}^{2 \pi} \int_{0}^{\pi}\left[\operatorname{Re} \mathrm{P} \mathrm{U}^{*}-\frac{\partial \psi_{0}^{*}}{\partial \mathrm{z}^{*}}\right]^{2} \mathrm{dx} \mathrm{x}^{*} \mathrm{dz}^{*} \\
& A E B=\frac{1}{\left(-R a_{1}\right)}\left[\frac{1}{2} \int_{0}^{2 \pi} \int_{0}^{\pi} P\left[\mathrm{~T}_{\mathrm{f}}^{*}+\mathrm{T}_{\mathrm{b}}^{*}\right]^{2} \mathrm{~d} \mathrm{x}^{*} \mathrm{~d} \mathrm{z}^{*}\right]
\end{aligned}
$$

in which $-\partial \psi_{b}^{*} / \partial z^{*}=u_{b}^{*}$ is the modification to the initial wind profile by the secondary flow, and $T_{f}^{*}$ is the dimensionless form for $T_{f}$ in (2.2). The definitions for KEB and AEB are valid provided that $\left|\partial \psi_{b}^{*} / \partial z^{*}\right|<\operatorname{Re} P\left|U^{*}\right|$ and $\left|T_{b}^{*}\right|<\left|T_{f}^{*}\right|$, which we confirmed for the cases examined in sections 5 and 6 . The following $\mathrm{Ra}>0$ energy rate equations are obtained from the partial differential system $(2.3)-(2.4)$ :

$$
\begin{aligned}
& \begin{array}{llll}
(\mathrm{HF}) & (\mathrm{RS}) & \left(\mathrm{K}_{\mathrm{r}}-\mathrm{MOD}\right) & \left(\mathrm{K}_{\mathrm{r}}-\mathrm{DIS}\right)
\end{array}
\end{aligned}
$$

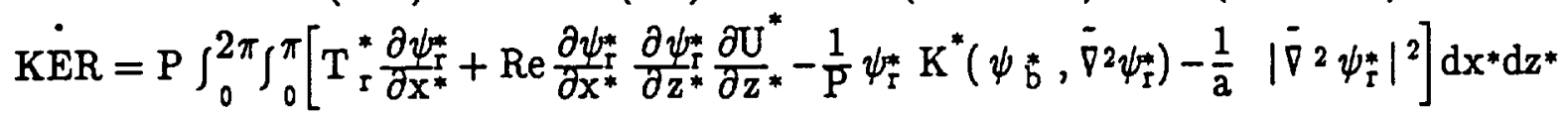

$$
\begin{aligned}
& -(\mathrm{HF}) \quad(\mathrm{GA}) \quad\left(\mathrm{A}_{\mathrm{r}}-\mathrm{MOD}\right) \\
& \dot{\mathrm{AER}}=\mathrm{P} \int_{0}^{2 \pi} \int_{0}^{\pi}\left[-\mathrm{T}_{\mathrm{r}}^{*} \frac{\partial \psi_{\mathrm{r}}^{*}}{\partial \mathrm{x}^{*}}+{ }_{-\mathrm{Ra}_{\mathrm{f}}} \mathrm{T}_{\mathrm{r}}^{*} \frac{\partial \psi_{\mathrm{r}}^{*}}{\partial \mathrm{x}^{*}}-\frac{1}{-\mathrm{Ra} \mathrm{a}_{\mathrm{l}}} \mathrm{T}_{\mathrm{r}}^{*} \mathrm{~K}^{*}\left(\psi_{\mathrm{r}}^{*}, \mathrm{~T}_{\mathrm{b}}^{*}\right)-\right. \\
& \left(A_{r}-D I S\right) \\
& \left.\frac{1}{-\mathrm{Ra}_{1} \mathrm{a}}\left|\tilde{\nabla} \mathrm{T}_{\mathrm{r}}^{*}\right|^{2}\right] \mathrm{dx} \mathrm{x}^{*} \mathrm{z}^{*}
\end{aligned}
$$


$-(\mathrm{RS}) \quad\left(\mathrm{K}_{\mathrm{b}}-\mathrm{MOD}\right) \quad\left(\mathrm{K}_{\mathrm{b}}-\mathrm{DIS}\right)$

$\dot{\mathrm{KEB}}=\mathrm{P} \int_{0}^{2 \pi} \int_{0}^{\pi}\left[-\operatorname{Re} \frac{\partial \psi_{\mathrm{r}}^{*}}{\partial \mathrm{x}^{*}} \frac{\partial \psi_{\mathrm{r}}^{*}}{\partial \mathrm{z}^{*}} \frac{\partial \mathrm{U}^{*}}{\partial \mathrm{z}^{*}}-\frac{1}{\mathrm{P}} \psi_{\mathrm{b}}^{*} \mathrm{~K}^{*}\left(\psi_{\mathrm{s}}^{*}, \bar{\nabla}^{2} \psi_{\mathrm{s}}^{*}\right)-\frac{1}{\mathrm{a}}\left|\bar{\nabla} 2 \psi_{\mathrm{b}}^{*}\right|^{2}\right] \mathrm{dx} \mathrm{dz}^{*}$

$-(\mathrm{GA}) \quad\left(\mathrm{A}_{\mathrm{b}}-\mathrm{MOD}\right) \quad\left(\mathrm{A}_{\mathrm{b}}-\mathrm{DIS}\right)$

$\dot{\mathrm{AEB}}=\mathrm{P} \int_{0}^{2 \pi} \int_{0}^{\pi}\left[--_{-\mathrm{Ra}_{1}} \mathrm{~T}_{\mathrm{r}}^{*} \frac{\partial \psi_{\mathrm{r}}^{*}}{\partial \mathrm{x}^{*}}-\frac{1}{-\mathrm{Ra}_{1}} \mathrm{~T}_{\mathrm{b}}^{*} \mathrm{~K}^{*}\left(\psi_{\mathrm{r}}^{*}, \mathrm{~T}_{\mathrm{r}}^{*}\right)-\frac{1}{-\mathrm{Ra_{1 }} \mathrm{a}}\left|\dot{\nabla} \mathrm{T}_{\mathrm{b}}^{*}\right|^{2}\right] \mathrm{dx} \mathrm{x}^{*} \mathrm{~d}^{*}$

When $\mathrm{Ra}<0$, we redefine AER and AEB in a typical way so that $(-\mathrm{Ra})$ replaces $\left(-\mathrm{Ra} \mathrm{a}_{1}\right)$ in the denominators of (3.2) and (3.4). In this case, the available potential energy rate equations simplify so that terms $-(\mathrm{HF})$ and (GA) combine to form only a heat flux term $-(\mathrm{HF})$ in (3.6), and the term $-(\mathrm{GA})$ is eliminated from (3.8).

In Appendix B, the energy rate equations (B1) - (B6) corresponding to the spectral system (A1) - (A14) are given for the case $\mathrm{Ra}>0$. These rate equations contain spectral representations of the terms labeled in (3.5) - (3.8) for the partial differential system. Since we retain only a few Fourier coefficients in the variable expansions $(2.9)-(2.10)$, the representations of these energy terms are only accurate to first order. As in (3.1) - (3.4), energy contributions generated by the roll perturbations are separated from those owing to the background flow so as to elucidate the nonlinear modifications of the initial environmental wind and temperature profiles. Each term contributes to the complete energy budget of the system, as is shown schematically in Fig. 2. In this diagram, the four energy pools are depicted, and in Table 1, each of the energy sources and sinks is described.

From Fig. 2 and Table 1, we see that rolls may extract background energy in the usual manner via both mechanical generation (RS) and, in statically unstable boundary layers, via thermal generation (GA); roll available potential energy (AER) is converted to roll kinetic energy (KER) in both stable and unstable boundary layers via vertical heat flux (HF). 
Conversely, the secondary flow may alter the initial background state via terms ( $\mathrm{K}_{\mathrm{b}}-\mathrm{MOD}$ ) $=-\left(K_{r}-M O D\right)$ and $\left(A_{b}-M O D\right)=-\left(A_{r}-M O D\right) ;$ typically, these terms are not displayed because the roll and roll modification components of the response are combined. Finally, terms (K-DIS) and (A-DIS) represent dissipation from each of the four pools of energy. The rate of change of total roll energy $E$ corresponding to the spectral system (A1) - (A14) may be written as

$$
\begin{aligned}
\dot{\mathrm{E}} & =\dot{\mathrm{KER}}+\dot{\mathrm{AER}} \\
& =[(\mathrm{RS})+(\mathrm{GA})]+\left[\left(\mathrm{K}_{\mathrm{r}}-\mathrm{MOD}\right)+\left(\mathrm{A}_{\mathrm{r}}-\mathrm{MOD}\right)+\left(\mathrm{K}_{\mathrm{r}}-\mathrm{DIS}\right)+\left(\mathrm{A}_{\mathrm{r}}-\mathrm{DIS}\right)\right]
\end{aligned}
$$

For steady energetics, bifurcations to temporally periodic roll solutions occur when the energy sources, given by (RS) and (GA), balance the energy sinks, given by ( $\left.K_{r}-M O D\right)$, ( $\left.A_{r}-M O D\right),\left(K_{r}-D I S\right)$ and $\left(A_{r}-D I S\right)$. Values for $E$ and for each of the individual energy terms are calculated in section $5 \mathrm{~b} 2$ from solutions to the nonlinear spectral model.

In the following section, we outline how we locate in $(\mathrm{Ra}, \mathrm{Re})$-parameter space the transition curves representing each of the possible roll instability modes. We also discuss the application of a higher resolution linear model used in the verification of the two-vertical wavenumber spectral model results.

\section{Linear stability analyses}

In this section we first outline the analytical linear stability analysis that is used to approximate the onset of roll modes in our spectral model, and then we discuss the more accurate numerical stability analysis of the linearized Boussinesq equations that is used to find an Ekman profile whose stability properties can be captured relatively well by our model. As noted by Stensrud (1987a), in both cases the roll modes of interest are found by a linear analysis of the motionless conductive solution. 


\section{a. Bifurcation equation for the roll modes}

As we noted in the introduction, a major advantage of use of low-order spectral models is that many of their solutions can be obtained analytically, thereby allowing a thorough exploration of roll behavior in forcing parameter space. Although linear stability analysis is a commonly used technique, the determination of the bifurcation equation governing the onset of the roll modes is somewhat complicated, and so we review briefly how we find this equation.

The linear system obtained from the spectral model (A1) - (A14) contains six equations, originating from (A5) - (A6) and (A11) - (A14), that are purely dissipative and so do not contribute to a change in stability. The remaining eight may be written as two sets of four complex equations in which one set is the conjugate of the other. Thus, only one set of four equations need be analyzed since it contains the same stability information as the other set (Pyle 1987). The form exp $\left[\lambda t^{*}\right]$ is assumed for a perturbation expressed in the new complex

variables $\bar{\psi}_{j}$ and $\overline{\mathrm{T}}_{\mathrm{j}}$; the real parts of the eigenvalues $\lambda$ indicate growth or decay of this perturbation and the imaginary parts represent its temporal periodicity. A nontrivial solution is found when the determinant of the matrix of coefficients vanishes, which in this case produces a complex fourth-order characteristic equation in $\lambda$ (Haack-Hirschberg 1988). Transitions to temporally periodic solutions are found by assuming that the real part of $\lambda$ vanishes and that the imaginary part of $\lambda$ is the dimensionless limiting frequency $\omega^{*}$ of the branching solution. These Hopf bifurcation points (e.g. Marsden and McCracken 1976; Pyle 1987) indicate critical values $\mathrm{Ra}_{\mathrm{c}}$ and $\mathrm{Re}_{\mathrm{c}}$ of the forcing rates beyond which propagating roll solutions develop. Minimum values of $R a_{c}$ and $R e_{c}$ provide the smallest forcing values needed for secondary instability and are presumably the first values reached as the atmosphere becomes unstable. 
Substitution of $\lambda=i \omega^{*}$ into the complex fourth-order characteristic equation yields two real equations in $\omega^{*}, \mathrm{Ra}_{\mathrm{c}}$ and $\mathrm{Re}_{\mathrm{c}}$. Upon combining these equations via the method of eliminants (Stensrud 1987b), we remove the frequency and obtain a Hopf bifurcation equation that is of sixth-order in $\mathrm{Ra}_{\mathrm{c}}$ (Haack-Hirschberg 1988). The coefficients of this equation were determined analytically with the program FORMAC on the PSU IBM $3090-600 \mathrm{~s}$. They are lengthy functions of $\mathrm{Re}_{\mathrm{c}}$, aspect ratio a, orientation angle $\beta$, vertical wavenumbers $q$ and $n$, Prandtl number $P$, and Fourier coefficients $\Lambda_{i}$ and $\Gamma_{i}$ of the basic wind. For a given arbitrary wind profile, we choose values for $q, n$ and $P$ and cycle over appropriate ranges for $\operatorname{Re}_{c}$, a and $\beta$. Minimum values of the critical forcing rates $\operatorname{Ra}_{c}$ and $\operatorname{Re}_{c}$ are found and these give preferred roll alignments $\beta_{\mathrm{p}}$ and aspect ratios $\mathrm{a}_{\mathrm{p}}$. From values of $\left(R a_{c}\right)_{\min },\left(R e_{c}\right)_{\min }, \beta_{p}$ and $a_{p}$, preferred values of the (real) dimensionless frequency magnitude $\left|\omega_{\mathrm{p}}^{*}\right|$ are also calculated; the magnitudes of the limiting dimensionless propagation rates of the rolls are given by $\left|{\underset{N p}{*}}_{p}^{*}\right|=\left|\omega_{\mathrm{p}}^{*}\right|$. Using the dimensional form $\omega=$ $\left[\left(2 \pi^{2} \kappa\right) /\left(\mathrm{z}_{\mathrm{T}} \mathrm{L}\right)\right] \omega^{*}$ of the frequency, where $\kappa$ is estimated to be $25 \mathrm{~m}^{2} / \mathrm{s}$ (e.g. Shirer and Brümmer 1986), and the definition $|\underset{N}{ }|=(L / 2 \pi)|\omega|$, we may obtain a preferred value $\left|c_{p}\right|$ $=\left(\pi \kappa / z_{\mathrm{T}}\right)\left|\omega_{\mathrm{p}}^{*}\right|$ for the phase speed. When these Hopf bifurcation points are displayed in ( $R a, R e)$-parameter space, transition curves for the various roll modes are produced (e.g. Shirer 1986; Stensrud 1987a); in section 5 we find that three principal modes are possible.

\section{b. Numerical analysis of the Boussinesq equations}

Although the bifurcation equation discussed above yields analytic approximations of the critical forcing rates and the preferred response parameters for the various roll modes, it only incorporates the effects of five of the Fourier coefficients of the initial background wind profile (cf. (2.1)). Because information in other Fourier terms of this wind profile may significantly affect the values of the forcing rates or the response parameters, a more 
thorough stability analysis must be done to ensure that the two-vertical wavenumber model (A1) - (A14) may be used successfully to examine the possible secondary flows created by a particular wind profile. This more detailed analysis can not be done analytically, thereby making a complete survey of parameter space tedious and computationally expensive.

The stability results produced by spectral expansions having two (2-WN), three (3-WN), and four (4-WN) vertical wavenumbers are obtained numerically and compared with those given by the Hopf bifurcation equation for the two-wavenumber model. The Fourier expansions of the basic wind profile have ten terms in the (3-WN) case and 17 terms in the (4-WN) case. Many of the terms in the $8 \times 8,12 \times 12$ and the $16 \times 16$ stability matrices, which are associated with the (2-WN), (3-WN) and (4-WN) representations respectively, contain spectral coefficients of the initial wind profile. However, because these coefficients involve at most only two of the vertical wavenumbers $q$ and $n$ (see Table A2), it is easy to specify the terms in these determinants merely by considering all combinations of the relevant wavenumber pairs in the general forms given in Haack-Hirschberg (1988). Because we normally find that the smallest wavenumbers give the best representation of an initial wind profile, we limit our more detailed analyses to combinations of $q=1$ and $n=2$ in the (2-WN) case, $q=1,2$ and $n=2,3$ in the ( $3-W N$ ) case, and $q=1,2,3$ and $n=2,3,4$ in the $(4-W N)$ case, where $q \neq n$.

To find the approximations of the roll mode transition curves given by the (2-WN) and the higher resolution ( $3-\mathrm{WN})$ and $(4-\mathrm{WN})$ representations, we must find all the eigenvalues of the above matrices for a large number of values for the parameters $\mathrm{Ra}, \mathrm{Re}$, a and $\beta$. Specifically, a point ( $\mathrm{Ra}, \mathrm{Re}$ ) on the transition curve as well as the associated preferred values of a, $\beta$ and $\omega^{*}$ are obtained as follows: The magnitude of Re is fixed and the value of $\mathrm{Ra}$ is gradually increased. For a particular value of $\mathrm{Ra}$, the values of a and $\beta$ are varied and the eigenvalue having the largest real part is saved in each case. The pair $(a, \beta)$ is found for which this largest real part reaches a maximum value. A preferred bifurcation point on the 
transition curve is obtained once the magnitude of $\mathrm{Ra}$ is increased sufficiently that this maximum value is zero.

If the values of the critical forcing and preferred response parameters do not vary significantly as additional wavenumbers are added to the problem, then we may conclude that a good representation for the instability modes has been obtained with only two vertical wavenumbers. Although to validate a particular wind profile we must investigate a large number of parameter values, we are guided somewhat in our choice of parameter ranges by the analytic results given by the Hopf bifurcation equation for the two-wavenumber model.

\section{Model results using the Ekman profile}

To facilitate comparisons with previous studies of idealized flow, we consider the stability results and nonlinear solutions forced by an Ekman spiral having Ekman depth D, where $\pi \mathrm{D} / 4$ is the height of maximum shear in the boundary layer. We define the dimensionless eastward and northward wind components for this profile as (Shirer 1986)

$$
\begin{gathered}
U_{s}^{*}\left(z^{*}\right)=\left|\underset{\sim g}{V_{g}^{*}}\right|\left[1-\exp \left(-z^{*} / D^{*}\right) \cos \left(z^{*} / D^{*}\right)\right] \\
V_{s}^{*}\left(z^{*}\right)=\left|\underset{\sim g}{V_{g}^{*}}\right| \exp \left(-z^{*} / D^{*}\right) \sin \left(z^{*} / D^{*}\right)
\end{gathered}
$$

where $\mathrm{D}^{*}=\mathrm{D} \pi / \mathrm{z}_{\mathrm{T}}$ and where $\left|\mathrm{V}_{\mathrm{g}}^{*}\right|$ is the magnitude of the dimensionless geostrophic wind given by the constraint $U_{s}^{*}(\pi)^{2}+V_{s}^{*}(\pi)^{2}=1$. We show below that an Ekman depth near $D^{*}$ $=1$ produces consistent stability results when more wavenumbers are included in the analysis. To obtain $\mathrm{D}^{*}=1$, we choose $\mathrm{D}=190 \mathrm{~m}$ and $\mathrm{z}_{\mathrm{T}}=600 \mathrm{~m}$; such a value of $\mathrm{z}_{\mathrm{T}}$ is typical of marine boundary layer depths (e.g. Brümmer 1985). Because the Ekman depth D is well below the inversion height $z_{\mathrm{T}}$, use of constant eddy viscosity $\nu$ and conductivity $\kappa$ is appropriate. We note that other authors (e.g. Faller and Kaylor 1966, 1967; Brown 1972; 
Asai and Nakasuji 1973) used infinitely deep domains and determined roll circulation depths from the solution itself. Despite these differences, we find below that our results using a $D^{*}=$ 1 Ekman profile agree rather well with these published results as well as with observations reported by LeMone (1973), LeMone and Pennell (1976) and Brümmer (1985).

Using the definitions in Table A2 with the above noted values $q=1$ and $n=2$, we calculate the values of the spectral coefficients $\Lambda_{\mathrm{i}}$ and $\Gamma_{\mathrm{i}}$ of the Ekman wind (5.1) $-(5.2)$; we also calculate the coefficients for the wavenumber pairs $(q, n), q \neq n$, given by $q=1,2,3$ and $n$ $=2,3,4$ that are needed in the validating numerical stability analysis. When the dimensionless form of (2.1) is substituted into the Fourier coefficient definitions (Table A2), expressions for the $U_{j}$ may be obtained to produce the following (2-WN) representation:

$$
\begin{array}{r}
\mathrm{U}^{*}\left(\mathrm{z}^{*}\right)=\left(\Lambda_{1}+\frac{1}{4} \Gamma_{1}+\frac{15}{16} \Gamma_{3}\right)-\frac{15 \pi}{32} \Gamma_{3} \sin \left(\mathrm{z}^{*}\right)+\frac{2}{3}\left(4 \Lambda_{2}+\Gamma_{2}\right) \cos \left(\mathrm{z}^{*}\right) \\
-\frac{5 \pi}{32}\left(\Lambda_{2}+\Gamma_{2}\right) \sin \left(2 \mathrm{z}^{*}\right)+\frac{1}{2}\left(\Gamma_{1}-\frac{5}{4} \Gamma_{3}\right) \cos \left(2 \mathrm{z}^{*}\right)
\end{array}
$$

We show in Fig. 3 the Fourier approximations calculated from (5.3) for the along-roll $V^{*}=$ $\mathrm{U}_{\mathrm{s}}^{*}$ and cross-roll $\mathrm{U}^{*}=-\mathrm{V}_{\mathrm{s}}^{*}$ wind components when $\beta=0^{\circ}$ (cf. Fig. 1). The solid curves represent the original Ekman wind profiles given by (5.1), (5.2) and (2.8), and the dashed curves show the approximated profiles given by (5.3). These results indicate that an excellent representation of the Ekman wind can be obtained with a Fourier series having as few as two vertical wavenumbers and five Fourier coefficients.

\section{a. Transition curves}

Having calculated values of the Fourier coefficients that reproduce the Ekman wind profile, we next use the methods described in section 4 to perform linear stability analyses of the conductive solution. Here and in the remaining sections, we set typical average values of $\nu=\kappa=25 \mathrm{~m}^{2} / \mathrm{s}(\mathrm{P}=1)$ based on computations by Shirer and Brümmer (1986). To obtain 
the minimum values of the forcing rates, we first find the minimum values of $R a_{c}$ and $R e_{c}$ given by solving the Hopf bifurcation equation of section 4a for appropriate ranges of the other parameters. The values of the aspect ratio $a$ and the orientation angle $\beta$ are varied first using a coarse resolution, from $0.1 \leq \mathrm{a} \leq 2.0$ in increments of 0.1 and from $-90^{\circ} \leq \beta \leq 90^{\circ}$ in increments of $10^{\circ}$, and then on a finer grid in which a is incremented every 0.05 and $\beta$ every degree. These results are then used to guide the higher resolution numerical analysis of section 4 b.

For the case $\mathrm{D}^{*}=1$, we compare in Fig. 4 a the transition curves for the twowavenumber (2-WN) (solid), three-wavenumber (3-WN) (dashed) and four-wavenumber (4-WN) (dotted) approximations obtained using the numerical method described in section 4b. It is this value of Ekman depth that yields the closest agreement among the three transition curves. Along these curves, the preferred values of a, $\beta$, and $\omega^{*}$ are given. We see clearly from the figure that the transition to secondary flow is well represented by the (2-WN) curve when Re is less than 60 or so, but that this curve begins to depart rapidly from the other two as the magnitude of Re is increased above 60. The (3-WN) and (4-WN) curves remain close for wider ranges of $\mathrm{Ra}$ and $\mathrm{Re}$. This result was found generally for a large number of Ekman depths and implies that a (3-WN) model may be more generally applicable (e.g. Stensrud and Shirer 1988). In the range $0 \leq \operatorname{Re} \leq 60$, the orientation angle results among the three approximations differ by at most $4^{\circ}$ and the aspect ratio values by no more than 0.1 , indicating very consistent results. Moreover, the response parameter values themselves compare well with those found by others who theoretically studied Ekman flow (e.g. Lilly 1966; Faller and Kaylor 1966; Brown 1970, 1972; Etling 1971; Asaj and Nakasuji 1973), and by those who observed the atmospheric boundary layer (e.g. LeMone 1973; Walter and Overland 1984; Brümmer 1985). For example, for modes near neutral stratification (Ra 0), we obtain the values $\mathrm{a}_{\mathrm{p}} \sim 0.6, \mathrm{~L}_{\mathrm{p}} \sim 2000 \mathrm{~m} \sim 10 \mathrm{D}, \beta \sim 10^{\circ}$ and $\left|\mathrm{c}_{\mathrm{p}}\right| \sim 0.2 \mathrm{~m} / \mathrm{s}$ that are consistent with those reported by the above authors. 
We conclude that for this Ekman profile, we may use the model (A1) - (A14) to study the modes developing when $\operatorname{Re}_{c} \leq 60$. However, we emphasize that this cutoff value is not a general one; each wind profile must be checked individually. We may also conclude that if the preferred orientation angles produced by using a roll-modified Ekman wind profile as the basic state differ by more than $4^{\circ}$ from those given by the pre-roll profile, then we have evidence that the errors obtained using observed profiles as basic states might be more likely attributable to the contamination of the observations by the roll solutions than to the limited truncation used to develop the model (A1) - (A14). We return to this point in section 6 .

In Fig. $4 \mathrm{~b}$ we show the transition curves obtained from the bifurcation equation for the two-wavenumber spectral model, as described in section 4a. Aside from minor differences in frequency - which are more accurately given by the bifurcation equation - these values are equivalent to those given in Fig. $4 \mathrm{a}$ for the (2-WN) numerical analysis of the linear model. In Fig. $4 \mathrm{~b}$ we separate the transition curve into the two principal modes: the thermal-q mode (dashed curve) that produces a solution dominated by wavenumber $\mathrm{q}=1$ and is associated with Rayleigh-Bénard convection when $U(z)=0$, and the inflection point mode (solid curve) whose transition curve passes through the neutral stratification value $\mathrm{Ra}=0$. A third mode was also found at large values of the Reynolds number $(\operatorname{Re}>100)$ and in strongly stable stratification $(\mathrm{Ra}<0)$; however, comparison of this dynamic mode with ones produced by the higher resolution models revealed that, while appearing in all three analyses, it nevertheless can not be well represented by our model. Thus, we restrict attention to values of Ra near those for neutral and unstable stratification.

As in Fig. 4a, the preferred values of the response parameters are given. We note that with increasing values of $\mathrm{Re}_{\mathrm{c}}$, the thermal-q mode is replaced by the inflection point mode near neutral stratification $(\mathrm{Ra}=0)$. For a variety of wind profiles, a smooth transition from the thermal-q to the inflection point mode occurs, indicating a possible link between these two instability mechanisms. Other thermal modes associated with wavenumbers $n=2$ and 
combinations of $\mathrm{q}$ and $\mathrm{n}$ are also common, but are not shown here because they occur at larger values of the forcing parameters and are therefore of little interest. Hereafter, we refer to the thermal-q mode as simply the thermal mode.

Two paths denoted by arrows and labeled with points $A$ and $B_{i}$ are drawn in Fig. 4 ; these paths, which we consider below, represent two possible evolutions of the atmosphere as the values of $R a$ and Re increase. In each case, the value of $R a>0$ is a statically unstable one so that the resulting circulation is both thermally and dynamically forced, and the value of $\operatorname{Re} \leq 60$ is in the range for which the model is valid (Fig. 4a). We summarize in Table 2 the minimum values $\left(\mathrm{Ra}_{c}, \mathrm{Re}_{c}\right)_{\min }$ of the critical forcing rates needed for roll development and the supercritical values $\left(\mathrm{Ra}_{c}, \mathrm{Re}_{c}\right)_{\text {sup }}$ of the forcing rates chosen at the labeled points. In section 6 , we ascertain how the rolls modify the initial profiles of wind and temperature by examining the effects of increasing the buoyant forcing, as given by increasing the value of $\mathrm{Ra}$ incrementally from point $B_{1}$ to $B_{2}$ to $B_{3}$ in Fig. $4 \mathrm{~b}$. In the following subsection, the solutions corresponding to points $A$ and $B_{i}$ are used to compare the roll stream function and perturbation temperature patterns, the energetics profiles, and the vertical fluxes of heat and momentum produced by the inflection point and thermal instability modes.

\section{b. Roll solutions}

To examine the nonlinear solutions given by the values of the forcing rates at points $\mathrm{A}$ and $B_{i}$ in Fig. $4 b$, we choose values of $a$ and $\beta$ near the preferred values on the transition curve closest to each supercritical point (see Table 2). We then numerically integrate the nonlinear equations (A1) - (A14). The rate of change of total roll energy is calculated from (3.9) using the definitions in Appendix B, and in all cases we find that energetically steady, temporally periodic roll solutions and steady background modifications occur. As noted earlier, the decay of the perturbations to a stable solution typically requires less than four 
hours, which justifies the neglect of the Coriolis terms. Figure 5 shows a schematic representation of the stream function pattern produced for a small, positive value of the preferred orientation angle $\beta_{\mathrm{p}}$. In this case, the roll circulations tilt downstream and propagate at a fixed amplitude in the $-x$ direction. The basic structure and tilt of these rolls are consistent with those shown, for example, by Faller and Kaylor (1966), Brown (1970, 1972) and LeMone and Pennell (1976).

\section{1). Stream function and perturbation temperature patterns}

At a fixed time, the dimensionless roll stream function patterns $\psi_{\mathrm{r}}^{*}($ from $(2.9))$ that correspond to the four points $A$ and $B_{i}$ are shown in Fig. 6 . All patterns propagate from right to left in the direction of the cross-roll wind (Fig. 3b). As the dynamic and thermodynamic forcing rates $R e$ and $R a$ are increased from point $B_{1}$ to $A$ (Fig. $6 \mathrm{~b}$ to $6 \mathrm{a}$ ), we note that the circulation tilt increases downwind in response to the greater wind speeds at the top of the domain (cf. (2.5)). The effects on the $\psi_{\mathrm{I}}^{*}$ fields of increasing only the thermal forcing rate Ra are shown in Figs. $6 \mathrm{~b}$ to $6 \mathrm{~d}$. Although the circulation patterns remain relatively unchanged, the corresponding dimensional maximum upward velocities increase from about 0.1 to $1.1 \mathrm{~m} / \mathrm{s}$. Qualitatively, these rolls have characteristics similar to those given by Brown's (1970) model of neutrally buoyant secondary flow (cf. his Fig. 11, in which propagation occurs from left to right) and by Brown's (1972) model of stratified flow (cf. his Figs. 8 and 9; propagation is also from left to right).

The dimensionless roll perturbation temperature patterns $\mathrm{T}_{\mathrm{r}}^{*}$ (from $(2.10)$ ) that correspond to the points $A$ and $B_{i}$ are shown in Fig. 7. In each case, positive temperature perturbations correspond to regions of upward motion in Fig 6. Direct thermal circulations are expected for cases of statically unstable stratification and produce the positive vertical heat fluxes shown below in Fig. 10a. As the thermal forcing rate $\mathrm{Ra}$ is increased from point 
$B_{1}$ to $B_{3}$ (Fig. $7 \mathrm{~b}$ to $7 \mathrm{~d}$ ), more efficient upward transports of heat are obtained by the reversal in the tilt of the $\mathrm{T}_{\mathrm{r}}^{*}$ field from upwind to downwind. Owing to the Boussinesq approximation, the temperature perturbation in our model best corresponds to either the density perturbation or the potential temperature perturbation in observations; the tilt in the temperature perturbation patterns in case $B_{3}$ most closely matches the observed cross sections through thermally forced rolls given by LeMone and Pennell (1976; cf. $\rho_{\mathrm{v}}$ in their Fig. 12) and Brümmer (1985; cf. $\theta_{\mathrm{R}}$ in his Fig. 7b).

Comparison of the roll solutions with LeMone's (1973, her Table 2) roll observations indicates that the circulation characteristics agree well with her values. LeMone (1973) estimated the maximum roll circulation speeds $u_{\max }$ to be between $0.077\left|{\underset{\sim}{g}}_{g}\right|$ and $0.15|\underset{\sim}{\mathrm{V}}|$. Using appropriate values in the expressions $(2.5),(5.1)$ and (5.2), we obtain magnitudes of $\mathrm{\sim}_{\mathrm{g}}$ for $R e=30$ and 60 . From the roll solutions we calculate values of $u_{\max }=|-\partial \psi / \partial z|_{\max }$ to be $0.07\left|\bigvee_{g}\right|$ for case $A$ and $0.19\left|\bigvee_{g}\right|$ for case $B_{1}$. Moreover, the cross-roll propagation velocity ${ }_{\sim}$, which ranges from -0.2 to $-0.3 \mathrm{~m} / \mathrm{s}$ (Table 2), is of the same sign and order of magnitude as the boundary layer average cross-roll wind velocity $\bar{U}$, in agreement with results usually found in observations (e.g. LeMone 1973) and models (e.g. Becker 1987; Chlond 1987). Thus, we conclude that the 14-coefficient spectral model represents rather well the essential characteristics of near-neutral two-dimensional secondary flow.

\section{2). Bnergy budgets and vertical transports}

The magnitude and sign of each energy term in (B1) - (B6) provide useful information about the relative importance of the mechanisms responsible for producing roll circulations and for modifying the initial wind and temperature profiles. These terms may be calculated from the solutions to the integrated spectral equations (A1) - (A14). First, values for the thermal surface forcing $\mathrm{Ra}_{\mathrm{f}}$ and the environmental stratification $\mathrm{Ra}_{1}$ must be obtained from 
the value of $\mathrm{Ra}$. Using the definition (2.6) and assuming $\mathrm{T}_{\mathrm{sa}}=16^{\circ} \mathrm{C}$ and near-neutral conditions $\left(\gamma_{d}-\gamma_{e}=0.1 \cdot \mathrm{C} / 600 \mathrm{~m}\right)$, we calculate a value of $\operatorname{Ra}_{1} \sim-10$. Then $\operatorname{Ra} a_{f}$ (and so $T_{1 b}$ ) is determined by subtracting $R a_{1}$ from the given value of $R a$.

For points $A$ and $B_{i}$ in Fig. 4b, we form energy budget diagrams in Fig. 8 showing the magnitudes of the sources, sinks and conversions of energy. Both a mechanical source of energy, via the Reynolds stress term (RS), and a thermal source of energy, via the generation term (GA), contribute to roll development. Consistent with the fact that $R a>0$, in all cases the heat flux term (HF) is positive so that energy is converted from roll available potential to kinetic forms. Comparing the magnitudes of the energy sources for points $A$ and $B_{1}$ reveals that mechanical generation is the larger source term for rolls driven by the inflection point instability mechanism (Fig. 8a), while thermal generation is the larger term for rolls excited by thermal instability (Figs. 8b,c,d). As the thermal forcing rate is increased from point $B_{1}$ to $B_{3}$ (Figs. $8 \mathrm{~b}$ to $8 \mathrm{~d}$ ), we note a marked increase in the magnitude of the (GA) term, as well as greater channeling of roll energy to the background flow via the modification terms $\left(K_{b}-M O D\right)$ and $\left(A_{b}-M O D\right)$. These roll modification terms become important at larger supercritical forcing rates. In case $B_{2}$ for example, the ratio of the modification terms to the source terms is roughly one-half. Thus we might expect that significant modification to the initial wind and temperature profiles would be possible once the thermal forcing rate is increased sufficiently.

Figure 9 shows vertical profiles for every term contributing to the energy budgets in Figs. 8a,b,c. Solutions for points A (inflection point mode), $B_{1}$ and $B_{2}$ (thermal modes) correspond to dashed, solid and dotted line types respectively. Energetics profiles for the supercritical point $B_{3}$ are omitted since they contain shapes consistent with those shown for $B_{1}$ and $B_{2}$, but with larger magnitudes. Asai and Nakasu $\ddot{j}$ (1973) present energetics profiles in their study of the stability of the Ekman boundary layer. Although their upper boundary is infinite, vertical profiles of Reynolds stress, generation and dissipation terms, 
corresponding to the inflection point and convective modes, agree well with those shown here. Brümmer's (1985) energetics analyses of KonTur data include Reynolds stress (RS) profiles that indicate a roll kinetic energy source in the lower half of the domain near the altitude of the inflection point in the cross-roll winds, as well as generation $(\mathrm{GA})$ profiles that reveal roll available potential energy in the middle of the domain where the magnitudes of the perturbations are greatest. Both of these results are in agreement with those shown here in Fig 9.

The dimensional profiles of the horizontally averaged, roll-induced vertical heat $\overline{\left(\mathrm{wT}^{\prime}\right)}$ and momentum (uw) fluxes are shown in Fig. 10. These flux profiles are consistent with those given by Asai and Nakasuj̈'s (1973) study of Ekman flow, by Etling and Raasch's (1987), Becker's (1987) and Chlond's (1987) higher resolution numerical studies of boundary layer circulations and by LeMone's (1973) and LeMone and Pennell's (1976) observational studies of roll vortices. That is, in a statically unstable environment, positive heat fluxes represent a stabilizing process in which the roll perturbations transport relatively warmer air upward and cooler air downward. Although most investigators find positive cross-roll momentum fluxes, our results in fact have a familiar form that is consistent with the downwind tilt of the roll circulations (Figs. 5 and 6). Because the changes $\partial U / \partial t$ in the initial cross-roll wind are given by $\partial U / \partial t=-\partial(\overline{u w}) / \partial z$ (e.g. Chlond 1987), the momentum flux profile should lead to an increase in the cross-roll wind speed below $300 \mathrm{~m}$ and a decrease above this level. This corresponds to down-gradient transports of cross-roll momentum, consistent with the results of Faller and Kaylor (1967) and Brown (1970), and with the results of section 6 . 


\section{Modifications to the basic state profiles}

We noted earlier that a fundamental characteristic of nonlinear roll circulations is that they can significantly alter the basic state profiles. Faller and Kaylor (1967) and Brown (1970) produce Ekman spirals that have been modified by the secondary flow. According to Brown (1980), roll modifications may change the basic flow by as much as 10 to $20 \%$. In each case, the altered flow has maximum velocities and is more closely aligned with the direction of the geostrophic wind at low levels, and becomes supergeostrophic at upper levels (Brown 1970). These results suggest that cross-roll modifications are channeled via the Coriolis force into the along-roll wind component, thereby increasing the roll-parallel flow and reducing the roll-perpendicular flow. In contrast, the numerical studies of Chlond (1987) and Etling and Raasch (1987) indicate that modification occurs predominantly in the cross-roll component of the basic wind profiles. Each of the above models differs from the present one by the inclusion of the longitudinal velocity component and Coriolis turning. Thus, their roll perturbations may alter the roll-parallel component of the initial basic wind profile, while only the roll-perpendicular component may be altered in the present model. We are therefore examining the roll modifications that can occur on a short time scale, before Coriolis turning can become significant. We find below that with increased supercritical forcing rates, the secondary flow decreases the cross-roll shear near the height of the inflection point.

For small supercritical forcing rates, such as those given by points $A$ and $B_{1}$ in Fig. $4 b$, the dimensional modifications to the initial background wind and temperature profiles are insignificant since they change these profiles by only a few percent. However, for the points $B_{2}$ and $B_{3}$ that are much farther to the right of the transition curve in Fig. $4 \mathrm{~b}$, the spectral components $\psi_{5}, \psi_{6}$ and $\mathrm{T}_{5}$ through $\mathrm{T}_{8}$ that represent modifications to the basic state increase significantly in magnitude. From points $B_{1}$ to $B_{3}$, the percentage of energy input that is 
converted to background energy, via terms $\left(K_{b}-M O D\right)$ and $\left(A_{b}-M O D\right)$, grows markedly, and so the rolls may begin to noticeably change the mean flow (Fig. 8). Although for short time scales the neglect of Coriolis forcing is a valid approximation, solutions far away from the transition curve may require more spectral modes than we have included in order to accurately resolve the nonlinear interactions that lead to a modified basic state. Therefore, we only examine the general structure and trends in these modified profiles, with emphasis on their potential effect on the results of a linear stability analysis.

Shown in Fig. 11 are the dimensional profiles of the perturbation temperature modification $T_{b}^{\prime}(z)$ (from (2.10)) for the solutions at points $B_{1}, B_{2}$ and $B_{3}$ (solid, dotted and dashed curves) on the lower path in Fig. $4 \mathrm{~b}$; because the initial temperature profile has a near-neutral lapse rate of approximately $9.6^{\circ} \mathrm{C} / \mathrm{km}$, the temperature modification shown is equivalent to that for potential temperature. As expected, the roll perturbations act to neutralize the interior portion of the unstably stratified boundary layer by creating more stable background temperature profiles; in each case the lower portion of the domain is cooled and the upper portion is warmed. Corresponding to case $B_{3}$ in Fig. 11, we use the roll-modified background lapse rate near $z=z_{T} / 2$ to calculate the modification to the thermodynamic forcing rate by the rolls. From its original value of $R a=50$, the thermal forcing rate is decreased to a near-neutral value of -1 . This result is consistent with the occurrence of direct thermal circulations and positive heat fluxes associated with thermally forced rolls. However, the magnitude of this temperature modification is not likely to be measurable in the atmosphere nor great enough to affect the linear stability results given in section $5 a$.

Conversely, alterations to the basic flow by the rolls do appear to be significant. The effects on the basic state wind profile of increasing the supercritical forcing rates are shown in Fig. 12. These profiles represent dimensional profiles of the initial background cross-roll wind $\mathrm{U}(\mathrm{z})$ (from (5.1), (5.2), (2.8)), the wind modification $\mathrm{u}_{\mathrm{b}}^{\prime}=-\partial \psi_{\mathrm{b}} / \partial \mathrm{z}$ (from (2.9)), and 
the modified cross-roll background wind $U+u_{b}^{\prime}$ for the solutions at points $B_{1}, B_{2}$ and $B_{3}$. We recall that the cross-roll component is determined from (2.8), where $\beta=6^{\circ}$ is the preferred roll orientation on the transition curve near point $B_{1}$ (Table 2). As shown in Fig. $12 \mathrm{~b}$, the roll perturbations yield profiles that have increasingly more negative slopes as the supercriticality increases from case $B_{1}$ to $B_{3}$; the mean wind direction is not changed, however. When summed with the original cross-roll Ekman profiles given in Fig. 12a, the modified background wind profiles in Fig. $12 \mathrm{c}$ are produced. As mentioned above, we conclude that these profile modifications are consistent with the momentum fluxes given in Fig. 10b. For the forcing rates corresponding to case $B_{3}$, the roll-induced shear is six times that of case $B_{1}$ and leads to substantial modification of the original basic flow. Consequently, the wind structure throughout the domain can be altered markedly by the roll circulations, even in the absence of the Coriolis force.

In Fig. 13, the dimensional original (solid) and case $B_{2}$ modified (dotted) Ekman spirals in roll coordinates are shown. As found by Faller and Kaylor (1967) and Brown (1970), the cross-roll wind speed of the modified profile is reduced significantly, so that the turning angle in the modified Ekman spiral is decreased. In addition, the modified spiral deviates less from the geostrophic wind at low levels than does the initial wind, producing a result that is also consistent with those of Brown (1970, his Fig. 14).

In theory, the wind profiles in Fig. 12c correspond to roll-modified winds that might be measured during cloud street observations. When values for the Fourier coefficients are calculated from these profiles, considerably different stability results might be obtained than those shown in Fig. 4b, for which the original, pre-roll Ekman profile was used. We examine this possibility by using the roll-modified background wind profile associated with point $\mathrm{B}_{3}$ to obtain the transition curves and preferred values of roll characteristics $\mathrm{a}_{\mathrm{p}}, \beta_{\mathrm{p}}$, $\omega_{\mathrm{p}}^{*}$ that are shown in Fig. 14. Comparing Figs. $4 \mathrm{~b}$ and 14, we note that only a thermal mode (dashed curve) may excite roll development in the range $0 \leq R e \leq 60$ for which the model is valid. 
Owing to the decreased shear near the inflection point in the modified Ekman profile, the inflection point mode disappears completely from the stability diagram. More importantly, the values of preferred orientation angle $\beta_{\mathrm{p}}$ differ between $10^{\circ}$ and $14^{\circ}$ from those shown in Fig. $4 \mathrm{~b}$ and indicate a larger than expected deviation (18 $)$ from the geostrophic wind direction. Such large changes are not entirely related to the use of a limited truncation because they are somewhat bigger than the changes shown in Fig. 4a between the (2-WN) and (4-WN) stability analyses of the original Ekman profile. In some cases involving observed wind profiles taken during cloud street events, use of the roll-modified winds leads to errors up to $35^{\circ}$ in the estimates of $\beta_{\mathrm{p}}$ (Shirer and Haack 1990). Significantly, these findings indicate that an appropriate amount of hypothesized roll-induced shear must be removed from an observed, cross-roll wind profile in order to determine the initial, basic state winds.

\section{Summary and conclusions}

In this study, a 14-coefficient nonlinear spectral model was used to investigate boundary layer roll circulations forced by Ekman flow. The Fourier representation of the wind, including two vertical harmonics and five Fourier coefficients, approximated the Ekman spiral rather well. Decay of small perturbations to stable roll solutions required less than four hours, which justified the neglect of Coriolis terms in the model. Thus, longitudinal sources of energy, and consequently the parallel instability mechanism, were eliminated. From the results of a linear stability analysis, characteristics of the convective and/or dynamic instability mechanisms were studied. This analysis was shown to be valid for $0 \leq \operatorname{Re} \leq 60$ by

comparing its results with those obtained from a numerical stability analysis of linear models having three and four vertical wavenumbers in the variable expansions.

The linear analysis produced transition curves in the forcing parameter space (Ra,Re) 
corresponding to each instability mechanism. Here Ra is the Rayleigh number that represents thermodynamic forcing from surface heating, and Re is the Reynolds number that represents dynamic forcing from the cross-roll wind shear. These curves corresponded to the minimum critical forcing rates beyond which stable roll solutions occur. In addition, preferred aspect ratios, frequencies, and roll axis alignments relative to the geostrophic wind were determined.

From the analysis of an Ekman wind profile having dimensionless Ekman depth $D^{*}=1$, we found three possible roll modes. The thermal or convective mode was only preferred for cases of unstable stratification ( $\mathrm{Ra}>0$ ) and weak dynamic forcing (small values of $\mathrm{Re}$ ). For near-neutral stratification ( $\mathrm{Ra} \sim 0$ ), the thermal mode was replaced by the inflection point mode. The preferred roll characteristics agreed well with those obtained from other boundary layer studies of Ekman flow (Faller and Kaylor 1966; Brown 1970; Etling 1971; Asai and Nakasuji 1973), and observational studies of roll vortices (LeMone 1973; LeMone and Pennell 1976; Brümmer 1985).

A new dynamic instability mechanism, the shear mode, occurred only in statically stable atmospheric conditions and for larger values of Re. Rapidly propagating rolls, with axes aligned at large angles to the mean wind direction and with small horizontal wavelengths, were excited by the shear instability. However, we concluded from comparisons with higher resolution numerical results that at least three vertical harmonics are required to model this new mode, and so it was not investigated further.

At supercritical values of the forcing rates, temporal integrations of the model equations yielded nonlinear solutions for the 14 spectral coefficients. These values were used to calculate the roll patterns and vertical transports corresponding to roll circulations driven by a particular instability mechanism. For all modes, the rolls tilted and propagated downwind at a constant rate and amplitude. All roll-induced fluxes were down-gradient and led to near-neutral stratification having reduced cross-roll shear. Energetics analyses indicated 
that roll circulations excited by the inflection point mode were predominantly driven by mechanical generation from Reynolds stress, while those excited by the thermal mode were driven predominantly by thermal generation from surface heating.

The alterations of the wind and temperature profiles were represented by six spectral coefficients in the model. For positive thermal forcing rates, the rolls modified the basic state temperature by cooling the lower, and warming the upper, portion of the domain. Dimensionally, however, this temperature change was not likely to be measurable in the atmosphere.

The rolls were shown to significantly alter the initial wind profile in the sense found by Faller and Kaylor (1967) and Brown (1970), but via a mechanism independent of the Coriolis force. Use of a roll-modified Ekman wind profile gave markedly different stability results that yielded errors of order $10^{\circ}$ in the preferred roll orientation angle $\beta_{\mathrm{p}}$. These errors are of the size reported by Shirer and Brümmer (1986), in which the roll modification to the winds was not considered. Even larger errors in $\beta_{\mathrm{p}}$ are possible, as demonstrated by Shirer and Haack (1990) who investigate the influence of the rolls on the observed basic wind by studying several hypothesized, pre-roll wind profiles for cases obtained during the stratocumulus phase of FIRE. 
Acknowledgments. We wish to thank Professor George S. Young for his comments on the various model assumptions and Dr. Harry W. Henderson and Mark J. Laufersweiler for their remarks on the energetics. In addition, the extensive, careful critique by Dr. Peggy LeMone and the comments by two other anonymous reviewers were very helpful. Lastly, we thank Jen-Ping Chen for assisting us with the formatting of the final manuscript. The research for this article was supported in part by the National Science Foundation through Grants ATM86-19854 and ATM89-09947, the National Aeronautics and Space Administration through Contract NAS8-36150 and Grant NAG8-780, and the Office of Naval Research through Contract N000014-86-K-06880. 
Appendix A

\section{The Nonlinear Spectral Model}

Here we list the 14 equations composing the nonlinear spectral model. The coefficients $a_{i}, b_{i}, c_{i}$ and $d_{i}$ are given in Table $A 1$, and the Fourier coefficients $\Lambda_{i}$ and $\Gamma_{j}$ in $c_{i}$ are defined in Table A2. The spectral system is given by

$$
\begin{aligned}
& \frac{\mathrm{d} \psi_{1}}{\mathrm{dt}}=-\mathrm{a}_{1} \psi_{4} \psi_{5}+\mathrm{a}_{2} \psi_{4} \psi_{6}+\mathrm{b}_{1} \mathrm{~T}_{1}+\operatorname{Re}\left(\mathrm{c}_{1} \psi_{2}+\mathrm{c}_{2} \psi_{4}\right)-\mathrm{Pd}_{1} \psi_{1} \\
& \frac{\mathrm{d} \psi_{2}}{\mathrm{dt}{ }^{*}}=a_{1} \psi_{3} \psi_{5}-a_{2} \psi_{3} \psi_{6}-b_{1} T_{2}-\operatorname{Re}\left(c_{1} \psi_{1}+c_{2} \psi_{3}\right)-P d_{1} \psi_{2} \\
& \frac{\mathrm{d} \psi_{3}}{\mathrm{dt} *}=-\mathrm{a}_{3} \psi_{2} \psi_{5}+\mathrm{a}_{4} \psi_{2} \psi_{6}+\mathrm{b}_{2} \mathrm{~T}_{3}+\operatorname{Re}\left(\mathrm{c}_{3} \psi_{4}+\mathrm{c}_{4} \psi_{2}\right)-\mathrm{Pd}_{2} \psi_{3} \\
& \frac{\mathrm{d} \psi_{4}}{\mathrm{dt} *}=a_{3} \psi_{1} \psi_{5}-a_{4} \psi_{1} \psi_{6}-\mathrm{b}_{2} \mathrm{~T}_{4}-\operatorname{Re}\left(\mathrm{c}_{3} \psi_{3}+\mathrm{c}_{4} \psi_{1}\right)-\mathrm{Pd}_{2} \psi_{4} \\
& \frac{\mathrm{d} \psi_{5}}{\mathrm{dt} *}=-\frac{\mathrm{a}_{5}}{2}\left(\psi_{1} \psi_{4}-\psi_{2} \psi_{3}\right)-\mathrm{P} \mathrm{d}_{3} \psi_{5} \\
& \frac{\mathrm{d} \psi_{6}}{\mathrm{dt} *}=-\frac{\mathrm{a}_{6}}{2}\left(\psi_{1} \psi_{4}-\psi_{2} \psi_{3}\right)-\mathrm{Pd}_{4} \psi_{6} \\
& \frac{d T_{1}}{d t^{*}}=-a_{6}\left(\psi_{5} \mathrm{~T}_{4}-\psi_{3} \mathrm{~T}_{5}\right)+\mathrm{a}_{5}\left(\psi_{3} \mathrm{~T}_{6}-\psi_{6} \mathrm{~T}_{4}\right)+\mathrm{a}_{7} \psi_{1} \mathrm{~T}_{7}+\mathrm{Ra} \psi_{1} \\
& -\operatorname{Re}\left(c_{5} T_{2}+c_{6} T_{4}\right)-d_{1} T_{1} \\
& \frac{d T_{2}}{d t^{*}}=-a_{6}\left(\psi_{4} T_{5}-\psi_{5} T_{3}\right)+a_{5}\left(\psi_{6} T_{3}-\psi_{4} T_{6}\right)-a_{7} \psi_{2} T_{7}-R a \psi_{2} \\
& +\operatorname{Re}\left(c_{5} T_{1}+c_{6} T_{3}\right)-d_{1} T_{2} \\
& \frac{\mathrm{dT}_{3}}{\mathrm{dt} \mathrm{t}^{*}}=-\mathrm{a}_{6}\left(\psi_{5} \mathrm{~T}_{2}-\psi_{1} \mathrm{~T}_{5}\right)+\mathrm{a}_{5}\left(\psi_{1} \mathrm{~T}_{6}-\psi_{6} \mathrm{~T}_{2}\right)+\mathrm{a}_{8} \psi_{3} \mathrm{~T}_{8}+\mathrm{Ra} \psi_{3} \\
& -\operatorname{Re}\left(c_{6} T_{2}+c_{7} T_{4}\right)-d_{2} T_{3} \\
& \frac{\mathrm{dT}_{4}}{\mathrm{dt}^{*}}=-\mathrm{a}_{6}\left(\psi_{2} \mathrm{~T}_{5}-\psi_{5} \mathrm{~T}_{1}\right)+\mathrm{a}_{5}\left(\psi_{6} \mathrm{~T}_{1}-\psi_{2} \mathrm{~T}_{6}\right)-\mathrm{a}_{8} \psi_{4} \mathrm{~T}_{8}-\mathrm{Ra} \psi_{4} \\
& +\operatorname{Re}\left(c_{8} T_{1}+c_{7} T_{3}\right)-d_{2} T_{4} \\
& \frac{d T_{5}}{d t^{*}}=\frac{a_{6}}{2}\left(\psi_{4} T_{2}+\psi_{2} T_{4}-\psi_{1} T_{3}-\psi_{3} T_{1}\right)-d_{3} T_{5} \\
& \frac{\mathrm{dT}_{6}}{\mathrm{dt}{ }^{*}}=\frac{\mathrm{a}_{5}}{2}\left(\psi_{2} \mathrm{~T}_{4}+\psi_{4} \mathrm{~T}_{2}-\psi_{1} \mathrm{~T}_{3}-\psi_{3} \mathrm{~T}_{1}\right)-\mathrm{d}_{4} \mathrm{~T}_{6} \\
& \frac{\mathrm{dT}_{7}}{\mathrm{dt} *}=\frac{\mathrm{a}_{7}}{2}\left(\psi_{2} \mathrm{~T}_{2}-\psi_{1} \mathrm{~T}_{1}\right)-\mathrm{d}_{5} \mathrm{~T}_{7}
\end{aligned}
$$




$$
\frac{\mathrm{dT}_{8}}{\mathrm{dt}^{*}}=\frac{\mathrm{a}_{8}}{2}\left(\psi_{4} \mathrm{~T}_{4}-\psi_{3} \mathrm{~T}_{3}\right)-\mathrm{d}_{6} \mathrm{~T}_{8}
$$

in which Re is the Reynolds number defined in (2.5), Ra is the Rayleigh number defined in (2.6), and $\mathrm{P}=\nu / \kappa$ is the Prandtl number. 


\section{APPENDIX B}

\section{Spectral Energetics ( $\mathrm{Ra}>0)$}

The following rate equations for the roll and background kinetic (KER), (KEB) and available potential (AER), (AEB) energies may be obtained from the spectral system (A1) -(A14). The individual terms are described in Table 1, and the corresponding rate equations for the partial differential system are given in $(3.5)-(3.8)$.

$$
\begin{aligned}
& \text { (HF) } \\
& \dot{\mathrm{KER}}=\mathrm{P}\left(\mathrm{T}_{1} \psi_{1}-\mathrm{T}_{2} \psi_{2}+\mathrm{T}_{3} \psi_{3}-\mathrm{T}_{4} \psi_{4}\right)+\operatorname{Re} \mathrm{P}\left[\Lambda_{2}\left(\mathrm{n}^{2} \mathrm{q}^{2}\right)\left(\psi_{1} \psi_{4}-\psi_{2} \psi_{3}\right)\right] \\
& \left(\mathrm{K}_{\mathrm{r}}-\mathrm{DIS}\right) \\
& +\left(K_{5}-M O D\right)-\frac{P}{a}\left[\left(a^{2}+q^{2}\right)^{2}\left(\psi_{1}^{2}+\psi_{2}^{2}\right)+\left(a^{2}+n^{2}\right)^{2}\left(\psi_{3}{ }^{2}+\psi_{4}{ }^{2}\right)\right] \\
& \dot{A E R}=-P\left(T_{1} \psi_{1}-T_{2} \psi_{2}+T_{3} \psi_{3}-T_{4} \psi_{4}\right)+\underset{-R_{1}}{R a_{1}} P\left(T_{1} \psi_{1}-T_{2} \psi_{2}+T_{3} \psi_{3}-T_{4} \psi_{4}\right) \\
& \text { (Ar-DIS) } \\
& +\left(A_{1}-M O D\right)-\frac{P}{-R a_{1} a}\left[\left(a^{2}+q^{2}\right)\left(T_{1}{ }^{2}+T_{2}{ }^{2}\right)+\left(a^{2}+n^{2}\right)\left(T_{3}{ }^{2}+T_{4}{ }^{2}\right)\right] \\
& \begin{array}{cc}
-(\mathrm{RS}) & \left(\mathrm{K}_{\mathrm{b}}-\mathrm{DIS}\right) \\
\mathrm{KEB}=-\operatorname{Re} \mathrm{P}\left[\Lambda_{2}\left(\mathrm{n}^{2}-\mathrm{q}^{2}\right)\left(\psi_{1} \psi_{4}-\psi_{2} \psi_{3}\right)\right]+\left(\mathrm{K}_{\mathrm{b}}-\mathrm{MOD}\right)-\frac{\mathrm{P}}{\mathrm{a}}\left[2(\mathrm{n}-\mathrm{q})^{4} \psi_{5}{ }^{2}+2(\mathrm{n}+\mathrm{q})^{4} \psi_{6}{ }^{2}\right]
\end{array} \\
& \dot{A E B}=-\sum_{-R a_{1}} P\left(T_{1} \psi_{1}-T_{2} \psi_{2}+T_{3} \psi_{3}-T_{4} \psi_{4}\right)+\left(A_{b}-M O D\right)- \\
& \left(\mathrm{A}_{\mathrm{b}}\right. \text {-DIS) } \\
& \frac{P}{-R_{1} a}\left[2(n-q)^{2} T_{5}^{2}+2(n+q)^{2} T_{6}^{2}+8 q^{2} T_{7}^{2}+8 n^{2} T_{8}^{2}\right]
\end{aligned}
$$

where the nonlinear modification terms are given by 


$$
\begin{aligned}
& \left(\mathrm{K}_{\mathrm{r}}-\mathrm{MOD}\right)=-\left(\mathrm{K}_{\mathrm{b}}-\mathrm{MOD}\right)= \\
& \quad \mathrm{a}_{5}\left(\psi_{5} \psi_{1} \psi_{4}-\psi_{3} \psi_{2} \psi_{5}\right)(\mathrm{q}-\mathrm{n})^{2}+\mathrm{a}_{6}\left(\psi_{6} \psi_{1} \psi_{4}-\psi_{6} \psi_{2} \psi_{3}\right)(\mathrm{q}+\mathrm{n})^{2}
\end{aligned}
$$

$$
\begin{aligned}
& \left(A_{r}-M O D\right)=-\left(A_{b}-M O D\right)= \\
& \frac{\mathrm{P}}{-\mathrm{R} \mathrm{a}_{1}}\left[\mathrm{a}_{5}\left(\mathrm{~T}_{6} \psi_{3} \mathrm{~T}_{1}-\mathrm{T}_{6} \psi_{4} \mathrm{~T}_{2}+\mathrm{T}_{6} \psi_{1} \mathrm{~T}_{3}-\mathrm{T}_{6} \psi_{2} \mathrm{~T}_{4}\right)\right. \\
& +a_{6}\left(\mathrm{~T}_{5} \psi_{3} \mathrm{~T}_{1}-\mathrm{T}_{5} \psi_{4} \mathrm{~T}_{2}+\mathrm{T}_{5} \psi_{1} \mathrm{~T}_{3}-\mathrm{T}_{5} \psi_{2} \mathrm{~T}_{4}\right) \\
& \left.+\mathrm{a}_{7}\left(\mathrm{~T}_{7} \psi_{1} \mathrm{~T}_{1}-\mathrm{T}_{7} \psi_{2} \mathrm{~T}_{2}\right)-\mathrm{a}_{8}\left(\mathrm{~T}_{8} \psi_{3} \mathrm{~T}_{3}-\mathrm{T}_{8} \psi_{4} \mathrm{~T}_{4}\right)\right]
\end{aligned}
$$

The coefficients $a_{\mathrm{i}}$ in (B5) - (B6) are given in Table A1. The dynamic and thermodynamic forcing parameters $\mathrm{Re}, \mathrm{Ra}_{1}$ and $\mathrm{Ra}_{\mathrm{f}}$ are defined in (2.5) and (2.6). The parameter $\mathrm{a}$ is the roll aspect ratio defined in (2.7), and $\mathrm{P}=\nu / \kappa$ is the Prandtl number. The Fourier coefficient $\Lambda_{2}$ is given in Table $A 2$, and $q$ and $n$ are vertical wavenumbers. 


\section{REFERENCES}

Asai, T., 1970a: Three-dimensional features of thermal convection in a plane Couette flow. J. Meteor. Soc. Japan, 48, 18-29. , 1970b: Stability of a plane parallel flow with variable vertical shear and unstable stratification. J. Meteor. Soc. Japan, 48, 129-139. , 1972: Thermal instability of a shear flow turning the direction with height. J. Meteor. Soc. Japan, 50, 525-532. , and I. Nakasuji, 1973: On the stability of Ekman boundary layer flow with thermally unstable stratification. J. Meteor. Soc. Japan, 51, 29-42.

Becker, P., 1987: Three-dimensional investigations of roll vortices: a case study. Contrib. Atmos. Phys., 60, 170-179.

Brown, R. A., 1970: A secondary flow model for the planetary boundary layer. J. Atmos. Sci., 27, 742-757. , 1972: On the inflection point instability of a stratified Ekman boundary layer. J. Atmos. Sci., 29, 850-859. ,1980: Longitudinal instabilities and secondary flows in the planetary boundary layer: a review. Rev. Geophys. Space Phys., 18, 683-697.

Brümmer, B., 1985: Structure, dynamics and energetics of boundary layer rolls from KonTur aircraft observations. Contrib. Atmos. Phys., 58, 237-254.

Chlond, A., 1987: A numerical study of horizontal roll vortices in neutral and unstable atmospheric boundary layers. Contrib. Atmos. Phys., 60, 144-169.

Dutton, J. A., and G. H. Fichtl, 1969: Approximate equations of motion for gases and liquids. J. Atmos. Sci., 26, 241-254.

Etling, D., 1971: The stability of Ekman boundary layer flow as influenced by the thermal stratification. Contrib. Atmos. Phys., 44, 168-186. 
, D., and S. Raasch, 1987: Numerical simulation of vortex roll

development during a cold air outbreak. Dyn. Atmos. Oceans, 10, 277-290.

Faller, A. J., and R. E. Kaylor, 1966: A numerical study of the instability of the laminar Ekman boundary layer. J. Atmos. Sci., 23, 466-480. , and __ 1967: Instability of the Ekman spiral with applications to the planetary boundary layers. Phys. Fluids Suppl., IUGG-IUTAM Symposium, 19-24 September, Kyoto, Japan, S212-S219. , and , 1969: Instability of the thermal wind. Clear Air Turbulence

and its Detection, Y. H. Pao and A. Goldburg (Eds.), Plenum Press, 63-72.

Haack-Hirschberg, T., 1988: Boundary layer roll circulations in a stratified atmosphere. M. S. thesis, available from Department of Meteorology, The Pennsylvania State University, University Park, PA., 161 pp.

Kaylor, R. and A.J. Faller, 1972: Instability of the stratified Ekman boundary layer and the generation of internal waves. J. Atmos. Sci., 29, 497-509.

Kelly, R.D., 1984: Horizontal roll and boundary-layer interrelationships observed over Lake Michigan. J. Atmos. Sci., 41, 1816-1826.

Kuettner, J. P., 1959: The band structure of the atmosphere. Tellus, 11, 267-294. 1971: Cloud bands in the earth's atmosphere: observations and theory. Tellus, 23, 404-425.

Kuo, H. L., 1963: Perturbations of plane Couette flow in stratified fluid and origin of cloud streets. Phys. Fluids, 6, 195-211.

Langmuir, I., 1938: Surface motion of water induced by wind. Science, 87, 119-123.

Laufersweiler, M. J., and H. N. Shirer, 1989: A simple dynamical model of a stratocumulus-topped boundary layer. J. Atmos. Sci., 46, 1133-1153. 
LeMone, M. A., 1973: The structure and dynamics of horizontal roll vortices in the planetary boundary layer. J. Atmos. Sci., 30, 1077-1091. , and W. T. Pennell, 1976: The relationship of trade wind cumulus distribution to subcloud layer fluxes and structure. Mon. Wea. Rev., 104, 524-539.

Lilly, D. K., 1966: On the instability of Ekman boundary flow. J. Atmos. Sci., $23,481-494$.

Lorenz, E.N., 1963: Deterministic nonperiodic flow. J. Atmos. Sci., 20, 130-141.

Marsden, J. E., and M. McCracken, 1976: The Hopf Bifurcation and its Applications. Applied Mathematical Sciences, 19, Springer-Verlag, 408 pp.

Mason, P.J. and R.I. Sykes, 1980: A two-dimensional numerical study of horizontal roll vortices in the neutral atmospheric boundary layer. Quart. J. R. Met. Soc., 106, 351-366. , and , 1982: A two-dimensional numerical study of horizontal roll vortices in an inversion capped planetary boundary layer. Quart. J. R. Met. Soc., 108, 801-823.

Pyle, R. J., 1987: Typical branching forms: periodic solutions. Nonlinear Hydrodynamic Modeling: A Mathematical Introduction, H. N. Shirer (Ed.), Lecture Notes in Physics, 271, Springer-Verlag, 264-291.

Shirer, H. N., 1980: Bifurcation and stability in a model of moist convection in a shearing environment. J. Atmos. Sci., 37, 1586-1602. 1986: On cloud street development in three dimensions: parallel and Rayleigh instabilities. Contrib. Atmos. Phys., 59, 126-149. (Ed.), 1987: Nonlinear Hydrodynamic Modeling: a Mathematical Introduction. Lecture Notes in Physics, 271, Springer-Verlag, 546 pp. 
, and B. Brümmer, 1986: Cloud streets during KonTur: a comparison of parallel/thermal instability modes with observations. Contrib. Atmos. Phys., 59, 150-161. , and T. Haack, 1990: The modification of large-scale wind profiles by roll circulations. Proc. Seventh Conf. Atmos. Rad., Amer. Met. Soc., J113-J118.

Sommeria, G., and M. A. LeMone, 1978: Direct testing of a three-dimensional model of the planetary boundary layer against experimental data. J. Atmos. Sci., 35, 25-39.

Stensrud, D. J., 1987a: The expected branching solution: preferred wavelengths and orientations. Nonlinear Hydrodynamic Modeling: A Mathematical Introduction, H. N. Shirer (Ed.), Lecture Notes in Physics, 271, Springer-Verlag, 292-324. , 1987b: The method of eliminants for finding common roots to polynomials. Nonlinear Hydrodynamic Modeling: A Mathematical Introduction, H. N. Shirer (Ed.), Lecture Notes in Physics, 271, Springer-Verlag, 514-516. , and H. N. Shirer, 1988: Development of boundary layer rolls from dynamic instabilities. J. Atmos. Sci., 45, 1007-1019.

Sun, W. Y., 1978: Stability analysis of deep cloud streets. J. Atmos. Sci., $35,466-483$.

Walter, B. A., and J. E. Overland, 1984: Observations of longitudinal rolls in a near neutral atmosphere. Mon. Wea. Rev., 112, 200-208.

Weston, K. J., 1980: An observational study of convective cloud streets. Tellus, 32, 433-438.

Woodcock, A. H., 1942: Soaring over the open sea, Sci. Mon., 55, 226-232. 


\section{FIGURE CAPTIONS}

Fig. 1. Rotation of the standard reference coordinate system $\left(x_{s}, y_{s}\right)$ into the roll coordinate system $(x, y)$. The cloud street orientation $\beta$ is the angle between the direction $x_{5}$ and the roll axis y (after Shirer 1980).

Fig. 2. Schematic energy budget diagram composed of roll kinetic (KER), roll available potential (AER), background kinetic (KEB), and background available potential (AEB) energies for the case $\mathrm{Ra}>0$. The individual terms are defined in the rate equations (B1) $-(B 6)$ and described in Table 1 . When $\mathrm{Ra}<0$, the generation term (GA) is eliminated.

Fig. 3. Dimensionless Ekman along-roll $\left(V^{*}=U_{s}^{*}\right)(a)$ and cross-roll $\left(U^{*}=-V_{s}^{*}\right)(b)$ wind components when $\beta=0^{\circ}$ for Ekman depth $D^{*}=1$. The solid curves are obtained from (5.1), (5.2) and (2.8), and the dashed curves from the Fourier representation (5.3).

Fig. 4 Curves of minimum critical forcing rates $\left(R a_{c}, R e_{c}\right)_{\min }$ for the Ekman profile (5.1) $-(5.2)$ when $D^{*}=1$. Values of the preferred aspect ratio $a_{p}$, orientation angle $\beta_{p}$ (in degrees) and frequency magnitude $\left|\omega_{\mathrm{p}}^{*}\right|$ (in parentheses) are labeled along each curve. In (a), the results using the two-wavenumber (2-WN) (solid), three-wavenumber (3-WN) (dashed) and four-wavenumber (4-WN) (dotted) numerical stability analyses of section $4 \mathrm{~b}$ are shown. In (b), the two-wavenumber results obtained from the Hopf bifurcation equation of section $4 \mathrm{a}$ are shown, with the dashed curve representing the thermal-q instability mode and the solid curve the inflection point instability mode. The arrows indicate two possible evolutions of the atmospheric forcing rates $\mathrm{Ra}$ and Re from subcritical to supercritical values; solutions at the points $A$ and $B_{i}$ are discussed in sections $5 b$ and 6 . The corresponding parameter values at each point are given in Table 2. 
Fig. 5. Schematic depiction of a propagating roll stream function pattern in the presence of an Ekman wind profile $\underset{\sim}{\bigvee}$ for a small, positive orientation angle $\beta_{\mathrm{p}}$. The axes $\left(\mathrm{x}_{\mathrm{s}}, \mathrm{y}_{\mathrm{s}}\right)$ represent the standard east/north coordinate system, and the axes $(x, y)$ represent the roll coordinate system. The parameter $z_{T}$ specifies the domain height, the roll spacing is given by the preferred wavelength $\mathrm{L}_{\mathrm{p}}$, and ${\underset{\sim}{\mathrm{g}}}_{\mathrm{g}}$ is the westerly geostrophic wind vector. Propagation is from right to left in the direction of the cross-roll flow.

Fig. 6. Patterns of dimensionless roll stream function $\psi_{r}^{*}$ for the points labeled $A(a), B_{1}(b)$, $B_{2}(c)$ and $B_{3}(d)$ in Fig. 4 b. Propagation is from right to left as denoted by the horizontal arrow. Parameter values associated with each point are given in Table 2.

Fig. 7. Patterns of dimensionless roll perturbation temperature $T_{r}^{*}$ for the points labeled $A(a), B_{1}$ (b), $B_{2}$ (c) and $B_{3}$ (d) in Fig. 4 b. Propagation is from right to left as denoted by the horizontal arrow. Parameter values associated with each point are given in Table 2.

Fig. 8. Dimensionless energy budget diagrams corresponding to the points labeled A (a), $B_{1}(b), B_{2}$ (c) and $B_{3}$ (d) in Fig. 4 b. Each term corresponds to a dimensionless energy rate defined in (B1) - (B6) and described in Table 1. The dimensionless roll energy is quantified by the value of $E$, and the boxes represent four separate pools of energy: roll kinetic (KER), roll available potential (AER), background kinetic (KEB), and background available potential (AEB). Parameter values associated with each point are given in Table 2. 
Fig. 9. Vertical profiles of each of the dimensionless energy rates defined in (B1) $-(B 6)$ for the points labeled $A, B_{1}$ and $B_{2}$ in Fig. $4 \mathrm{~b}$. Dashed curves correspond to the supercritical point for the inflection point mode (point $A$ ), solid curves for the thermal mode (point $B_{1}$ ), and dotted curves for the thermal mode (point $B_{2}$ ). The boxes (KER), (AER), (KEB) and (AEB) represent the four pools of energy. Parameter values associated with each point are given in Table 2.

Fig. 10. Vertical profiles of dimensional vertical (a) heat and (b) momentum fluxes for the points labeled $A, B_{1}$ and $B_{2}$ in Fig. 4 b. Line types are as in Fig. 9. Parameter values associated with each point are given in Table 2.

Fig. 11. Vertical profiles of the perturbation temperature modification $T_{b}^{\prime}$ for the solutions at the points labeled $B_{1}$ (solid), $B_{2}$ (dotted) and $B_{3}$ (dashed) in Fig. 4b. Parameter values associated with each point are given in Table 2.

Fig. 12. Vertical profiles of the initial cross-roll wind $U(a)$, the wind modification $u_{b}(b)$, and the modified background cross-roll wind $U+u_{b}^{\prime}(c)$, for the solutions at the points labeled $B_{1}$ (solid), $B_{2}$ (dotted) and $B_{3}$ (dashed) in Fig. $4 \mathrm{~b}$. Parameter values associated with each point are given in Table 2.

Fig. 13. Dimensional original and modified Ekman spirals and the geostrophic wind vector $\mathrm{V}_{\mathrm{g}}$ in roll coordinates. The solid curve is from the original wind components (5.1) $-(5.2)$ and the dotted curve from the modified winds shown in Fig. 12c corresponding to case $B_{2}$. Parameter values associated with this point are given in Table 2. 
Fig. 14 Curves of minimum critical forcing rates $\left(R a_{c}, R e_{c}\right)_{\min }$ obtained from the Hopf bifurcation equation of section $4 \mathrm{a}$ for the modified cross-roll Ekman wind profile shown in Fig. $12 c$ corresponding to point $B_{3}$. The dashed curve represents the thermal-q instability mode. Values of preferred aspect ratio $a_{p}$, orientation angle $\beta_{p}$ (in degrees), and frequency magnitude $\left|\omega_{\mathrm{p}}^{*}\right|$ (in parentheses) are labeled along the curve. Parameter values associated with this point are given in Table 2 . 


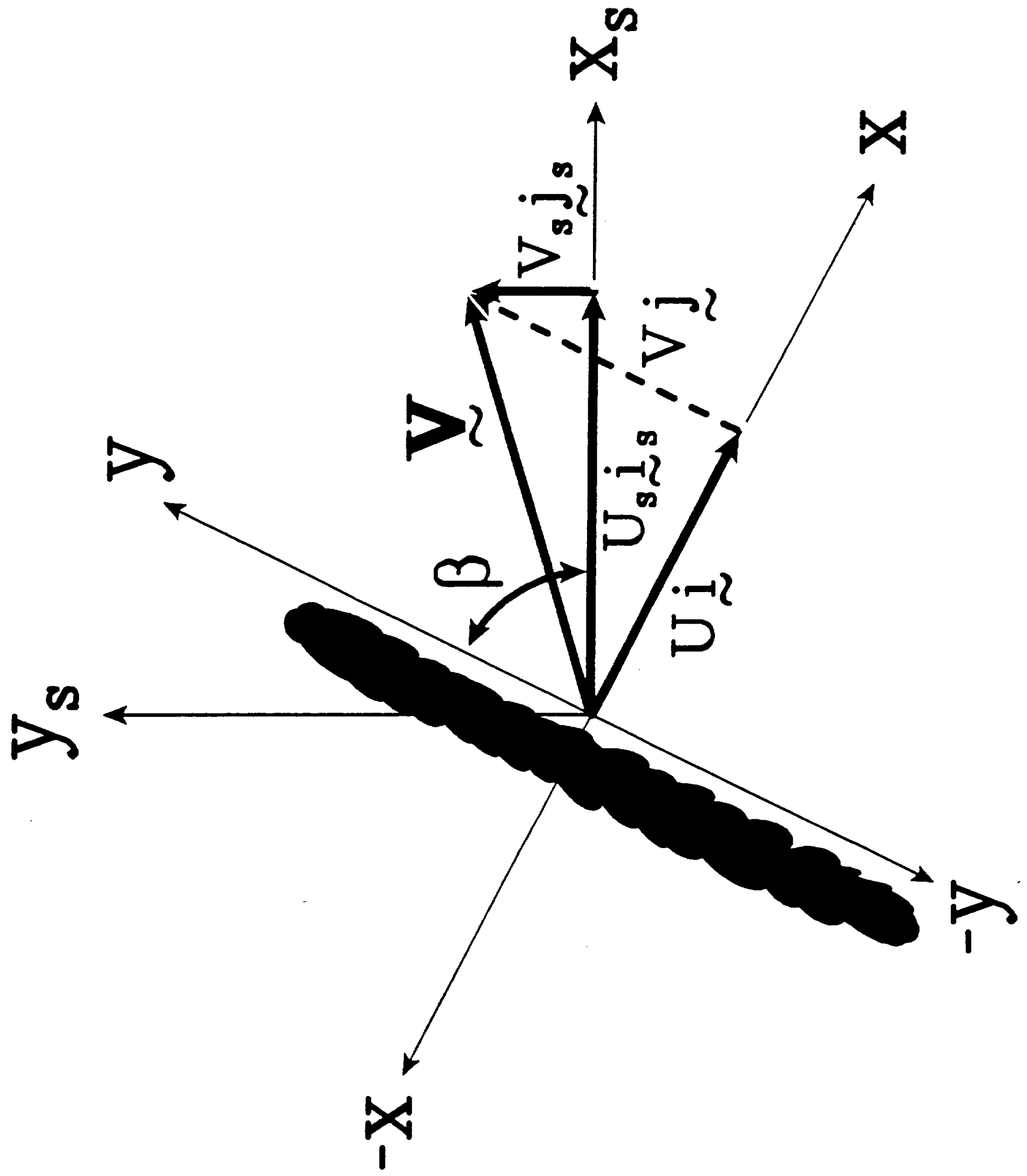

FIGURE 1 


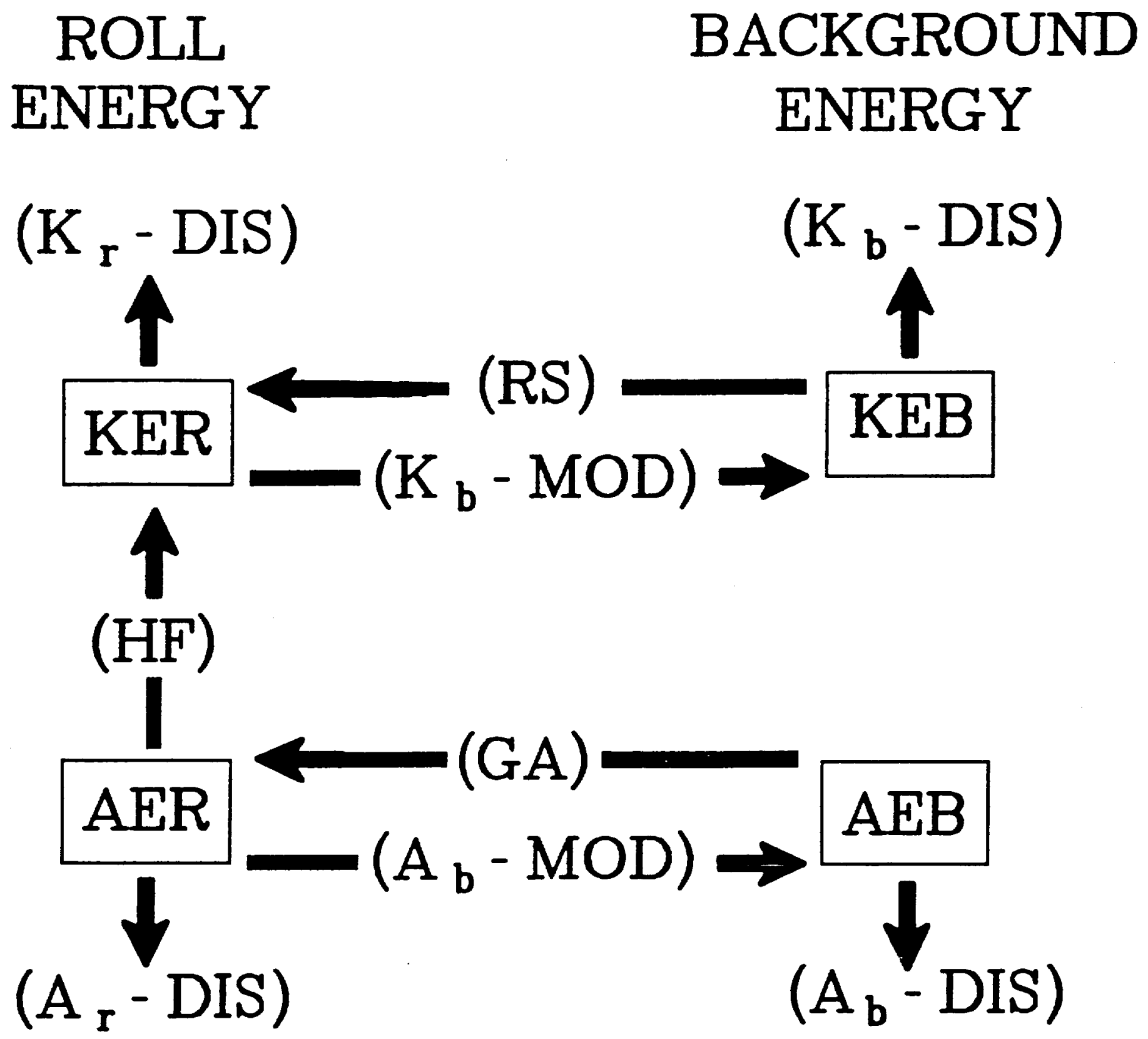



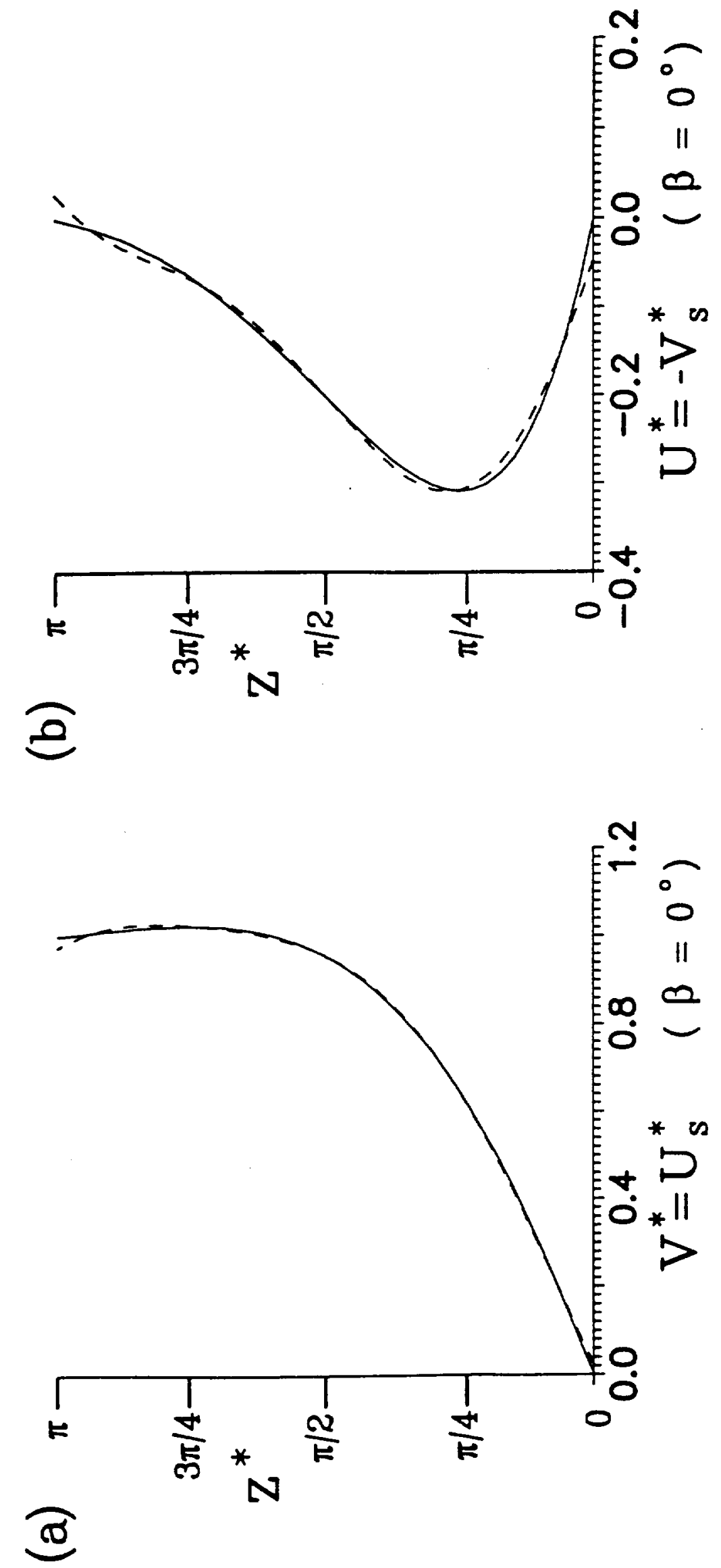
(a)

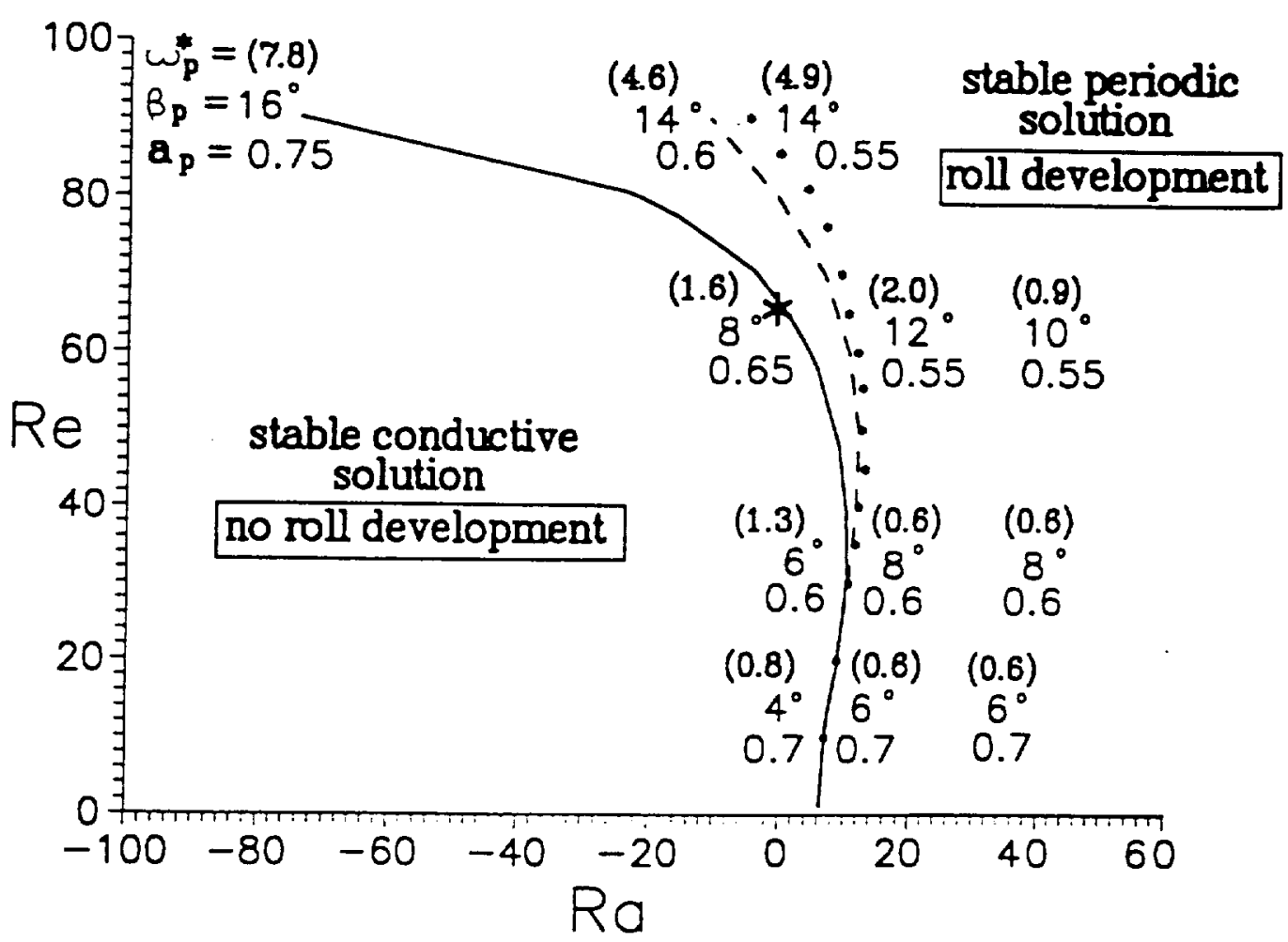

(b)

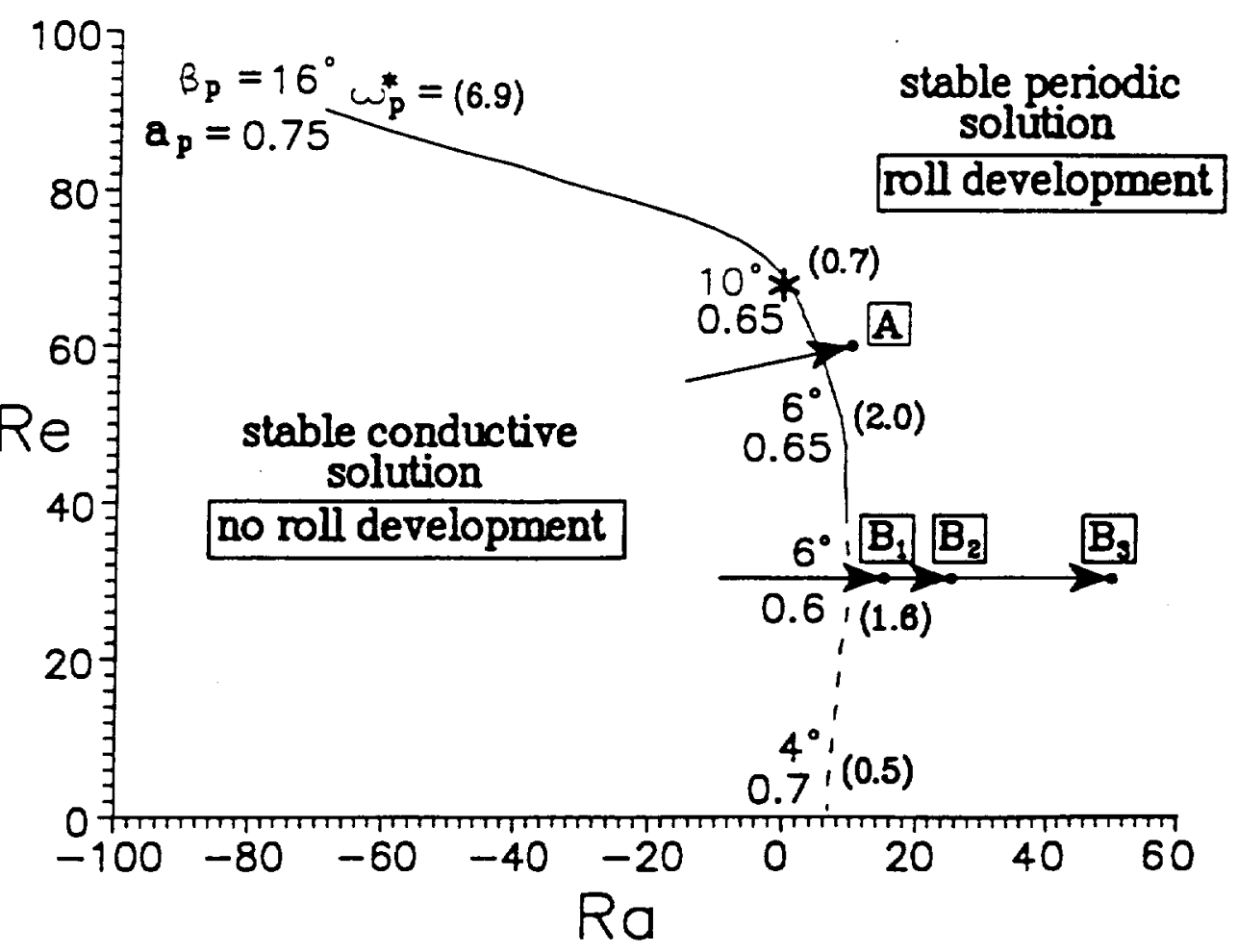




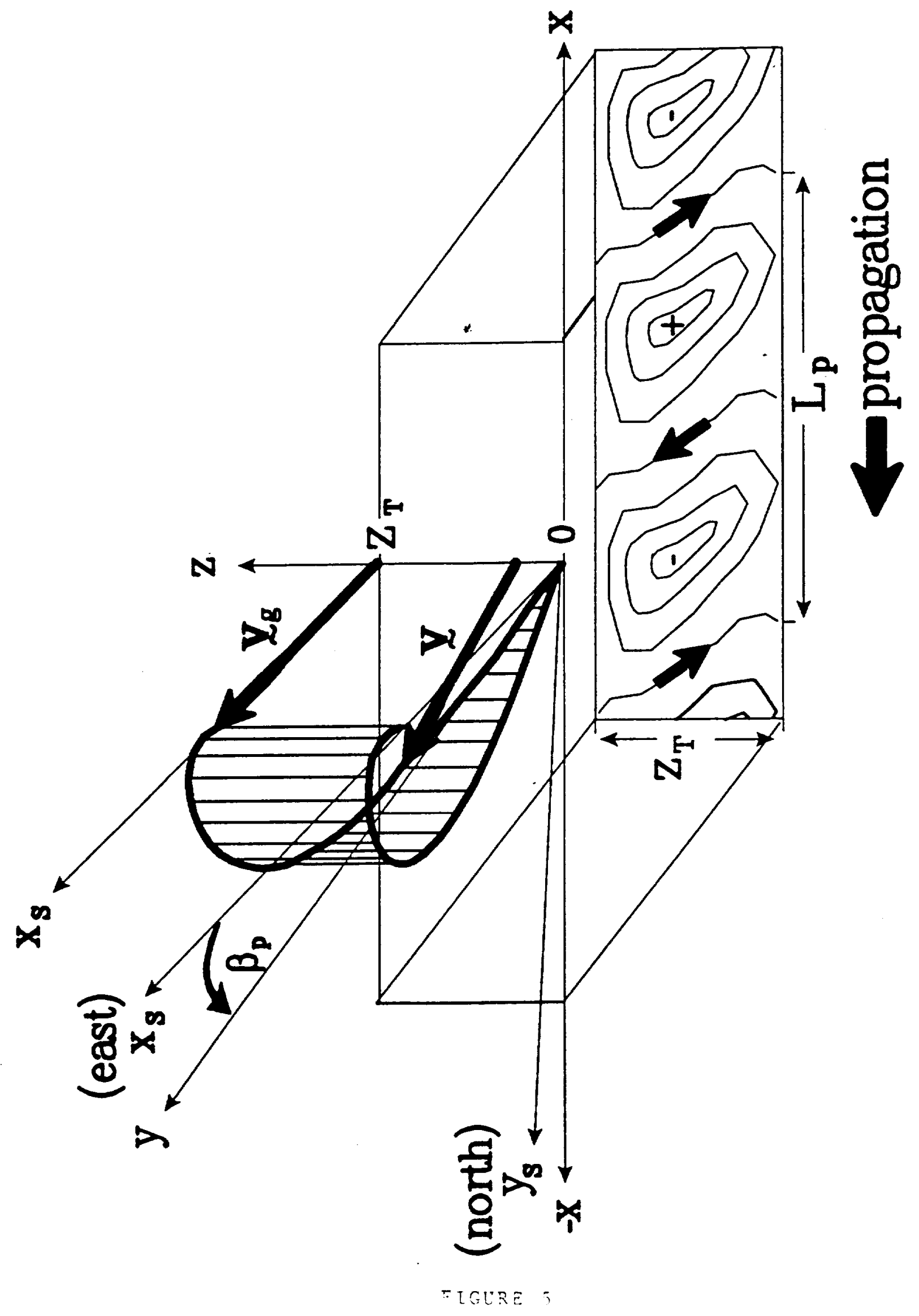



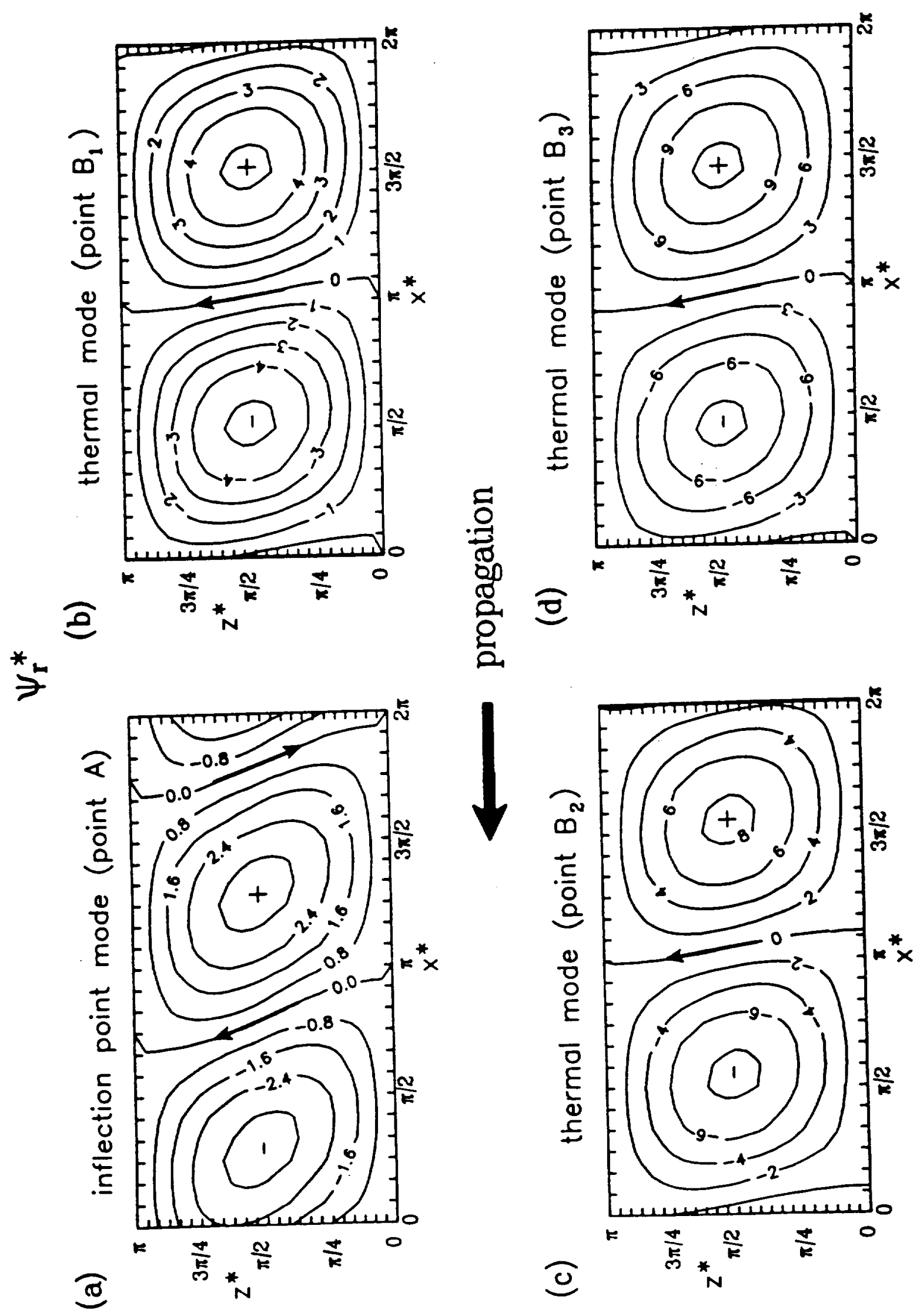

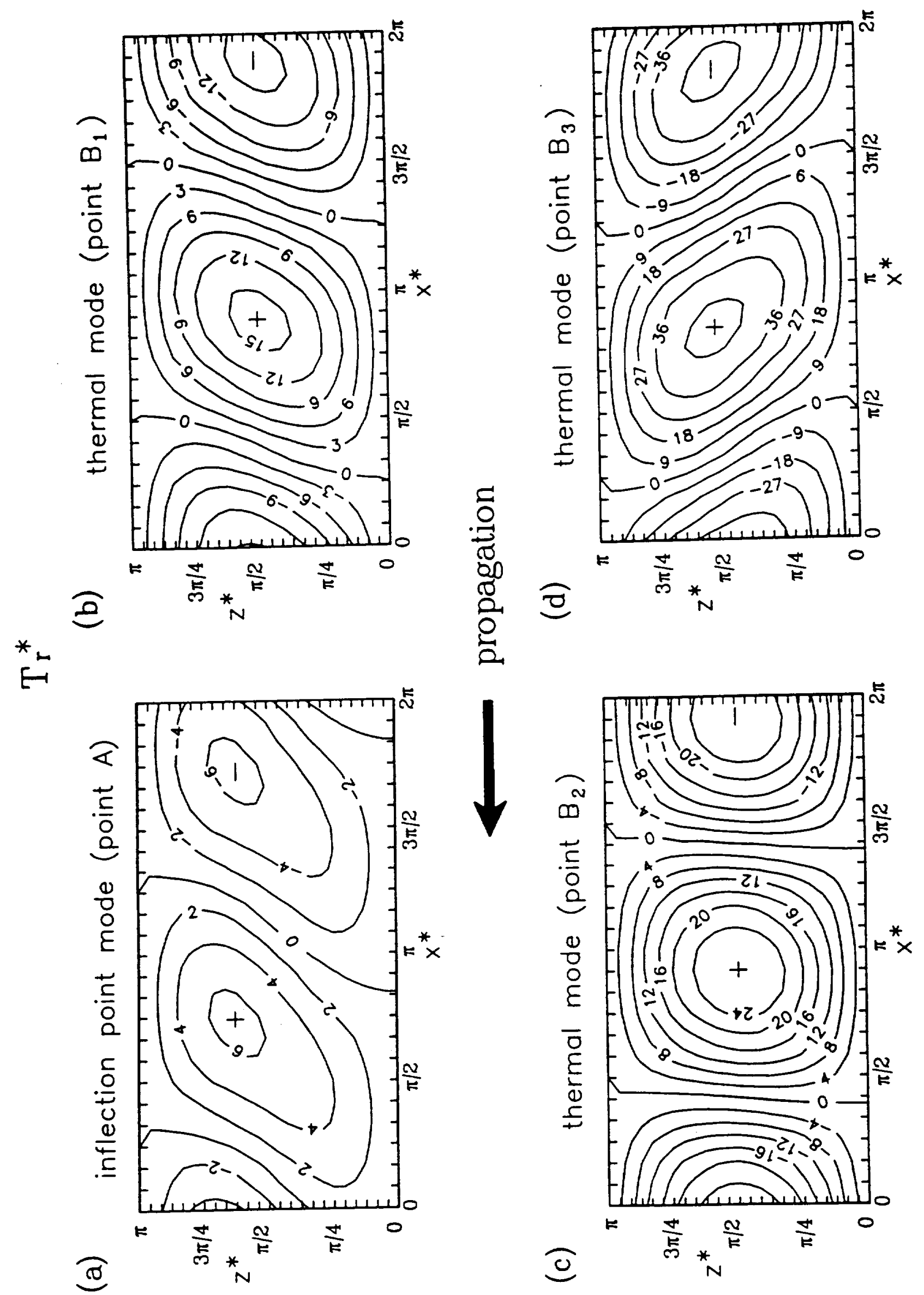

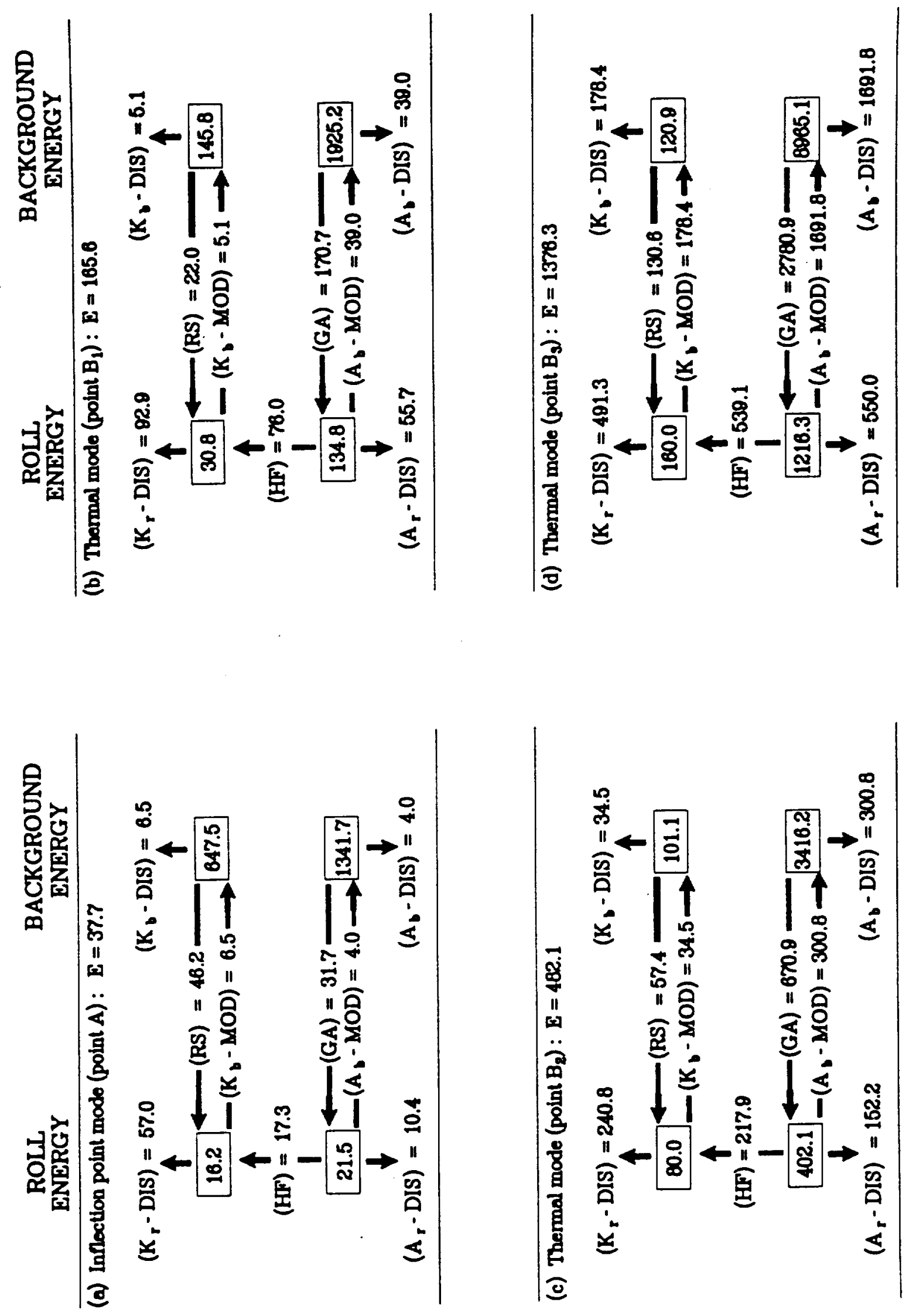


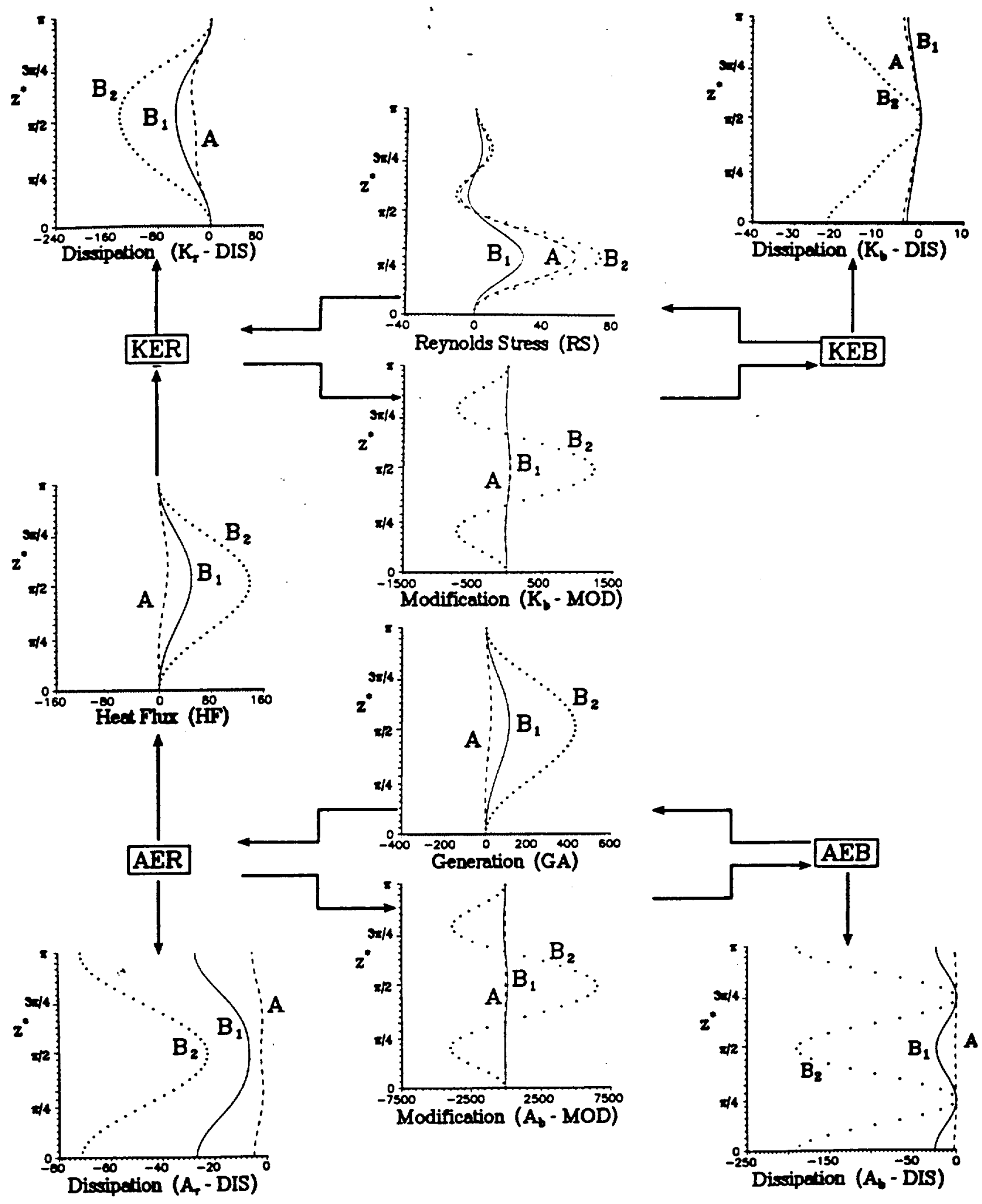



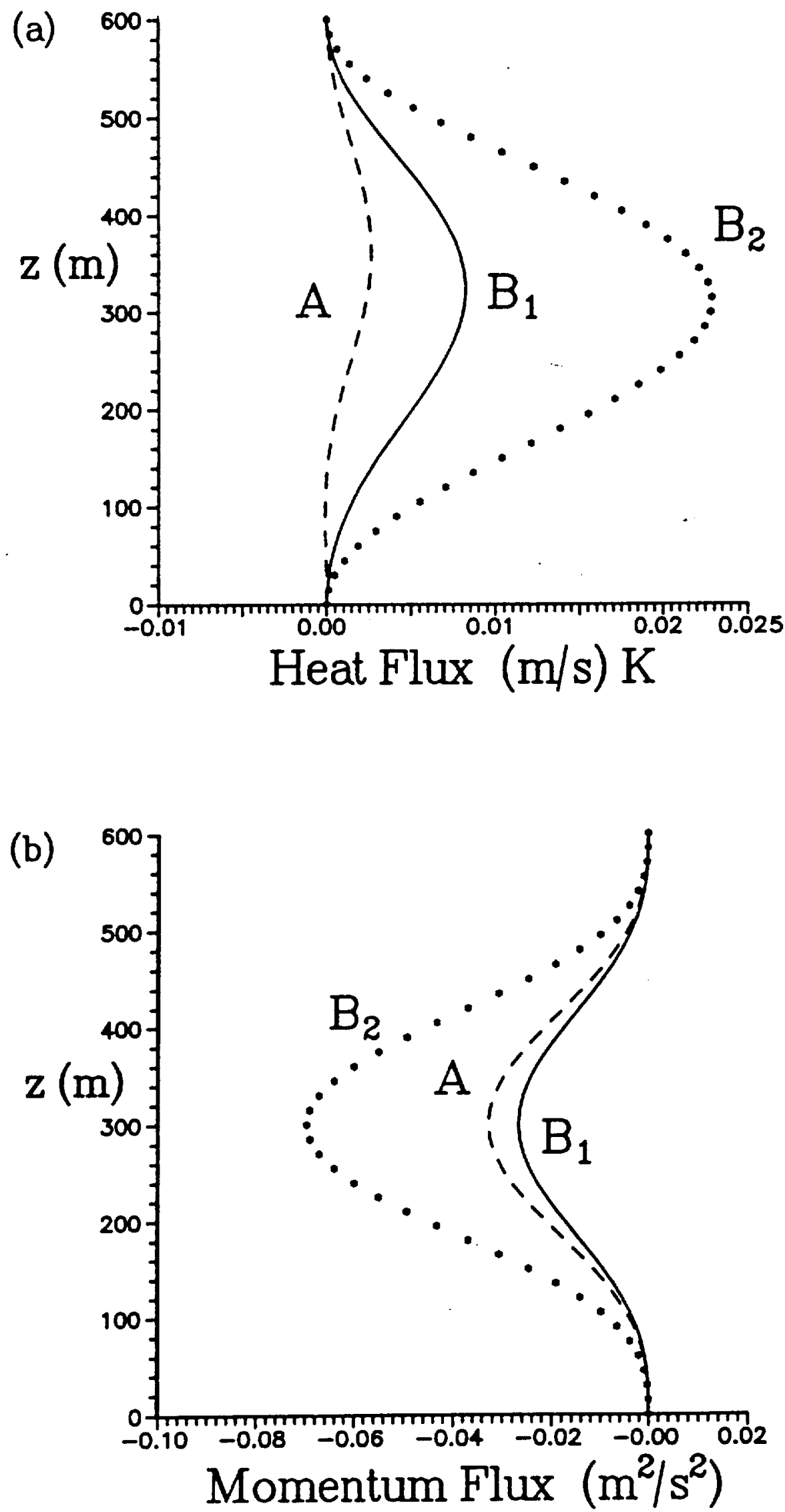


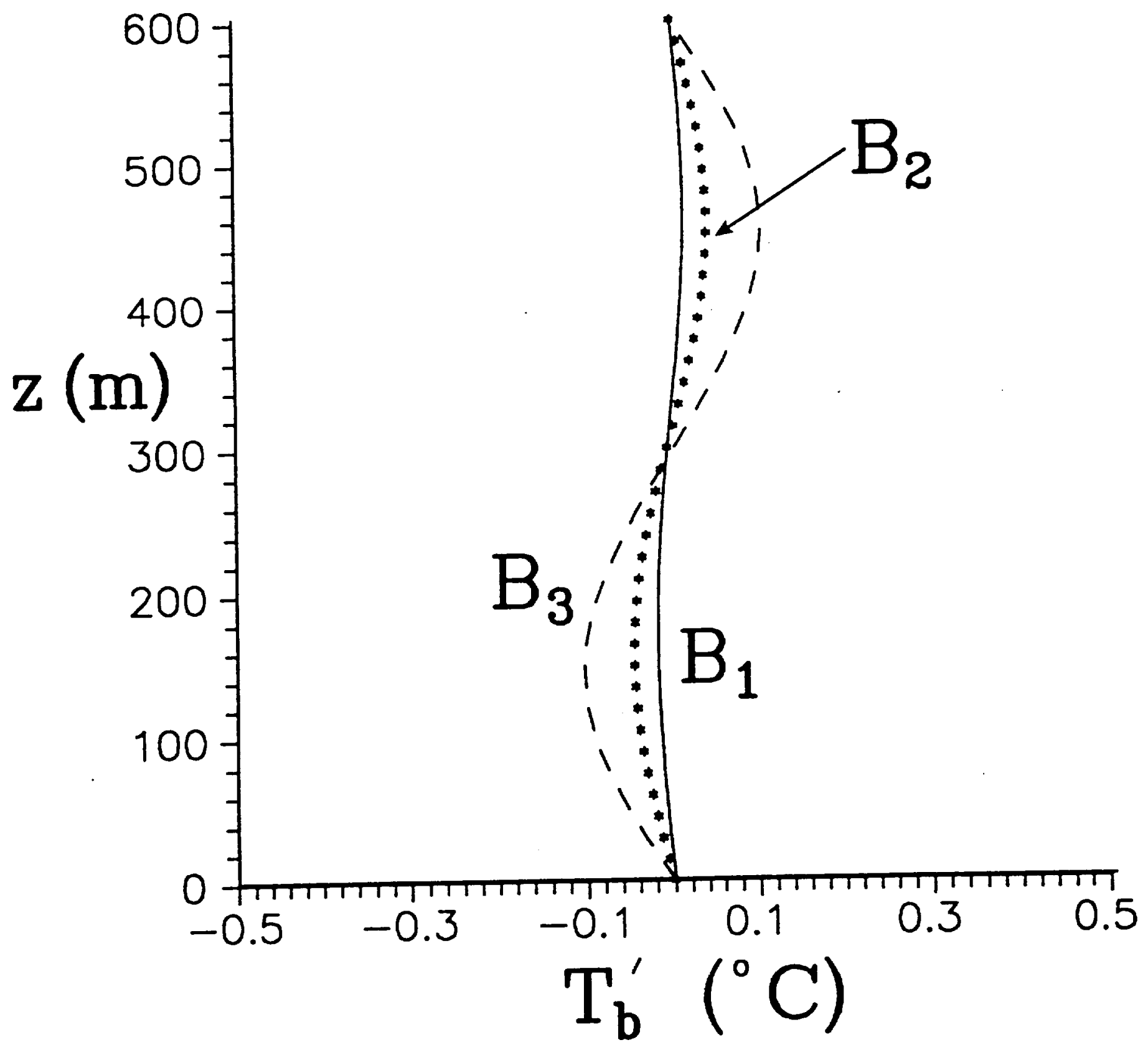



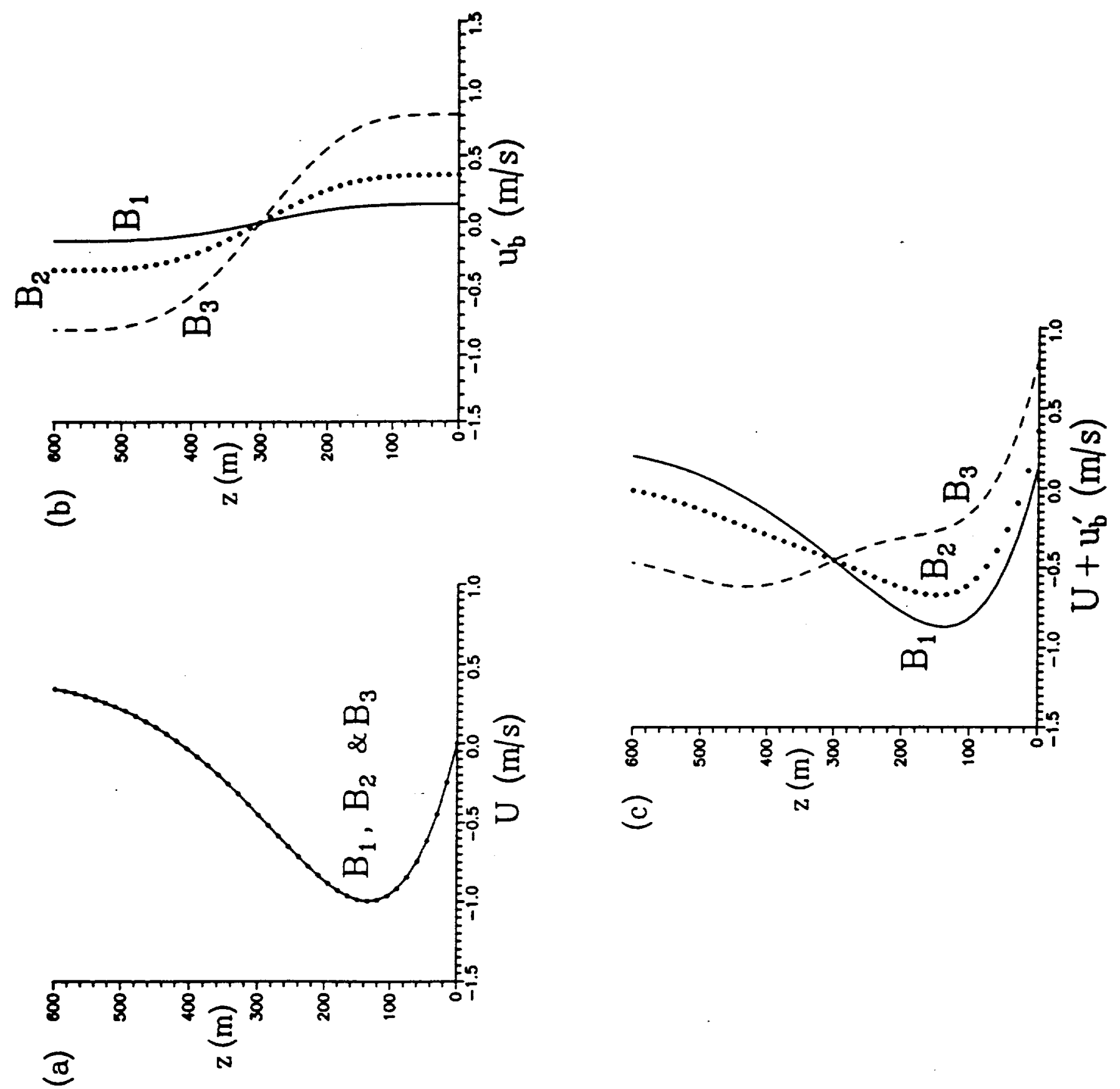


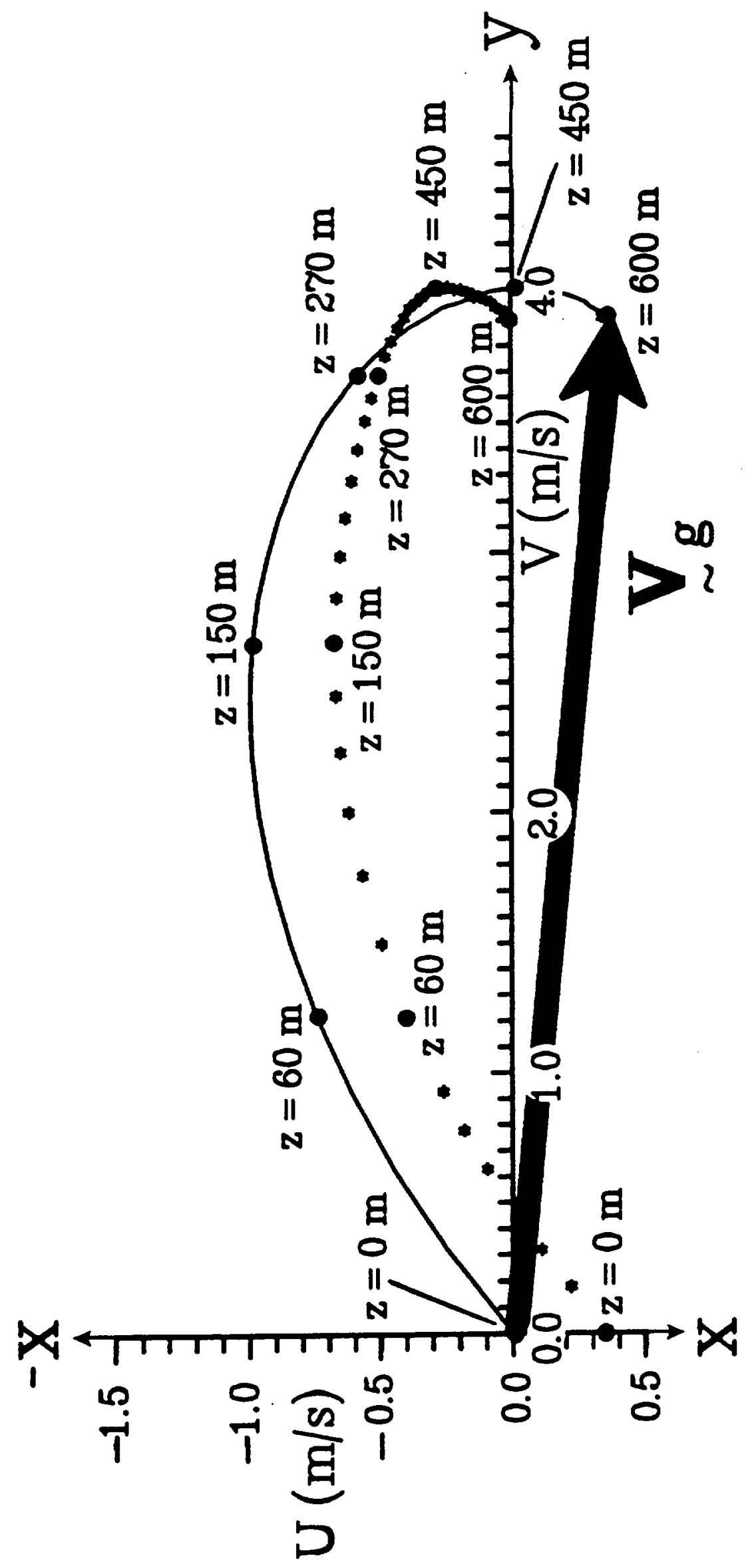

FIGURE : 


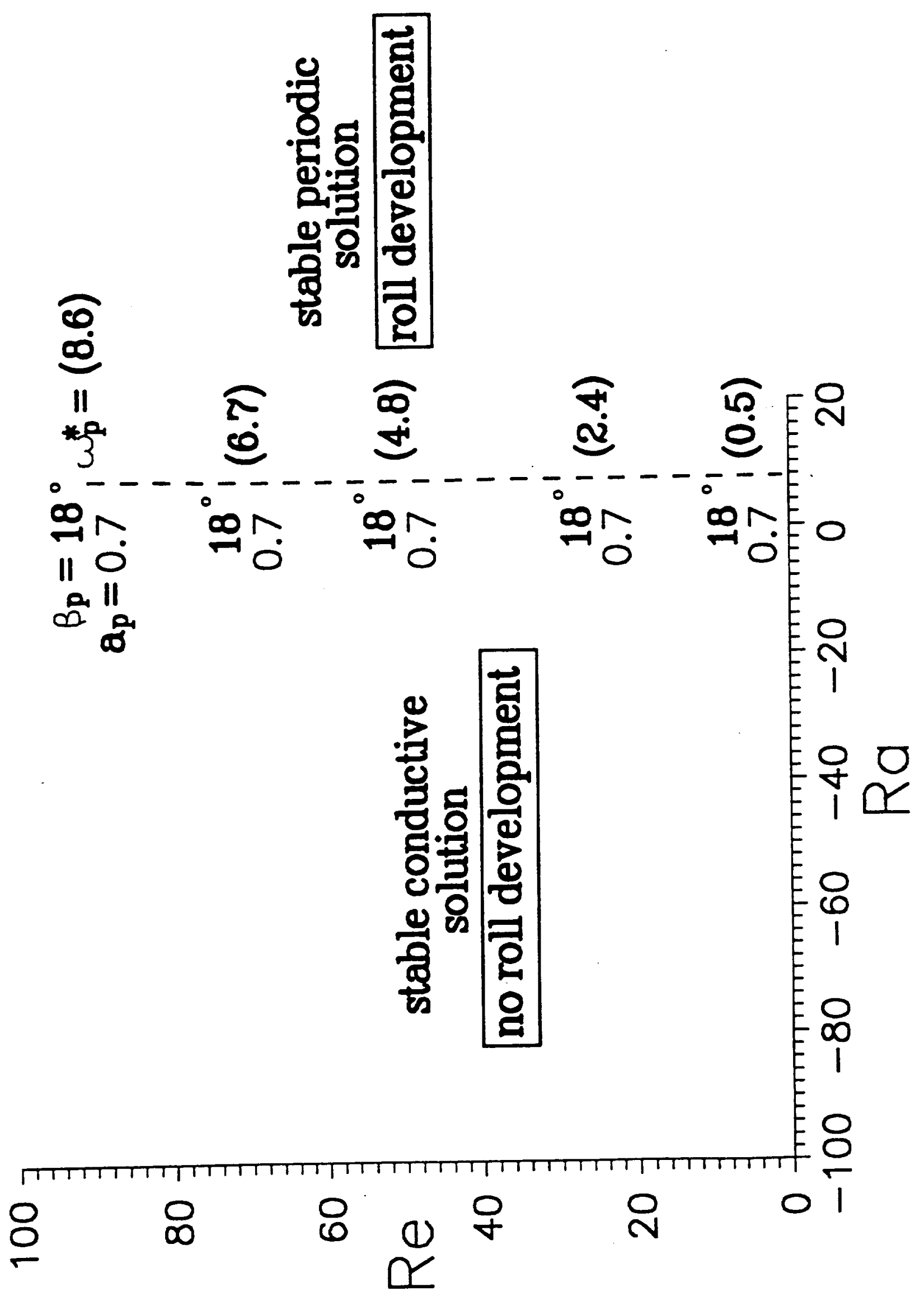


Table 1

Summary of energetics terms in (3.5) - (3.8).

$\begin{array}{ccccc}\text { TERH } & \text { DEFINITION } & \underline{\text { ORIGINATING TERMS }} & \underline{\text { SOURCE }} & \text { SINK } \\ \mathrm{HF} & \text { vertical heat flux or } & \partial \mathrm{T}^{*} / \partial \mathrm{x}^{*} \text { in }(2.3) \text { and } & \text { KER } & \text { AER } \\ & \text { AER/KER conversion } & \partial \psi^{*} / \partial \mathrm{x}^{*} \text { in }(2.4) & \end{array}$

RS Reynolds stress or advection terms involving KER REB mechanical generation $\sigma^{*}$ in $(2.3)$

GA thermal generation $\left(\mathrm{T}_{1 \mathrm{~b}}-\mathrm{T}_{\mathrm{s} a}\right)$ term of $\mathrm{Ra}$ AER AEB $(\mathrm{Ra}>0)$; or zero $(\mathrm{Ra} \leq 0) \quad$ in $(2.4)$; with reformulated AER and or none AEB rate equations

$\mathbf{K}_{\mathrm{b}-\text { HOD }}$ initial basic state Jacobian term in (2.3) KEB $\quad$ KER wind profile modification

$A_{b}$ - MOD initial basic state Jacobian term in (2.4) AEB AER temperature profile modification

K-DIS roll and background last term in (2.3) - - - KER viscous dissipations $\quad$ KEB

A-DIS roll and background last term in (2.4) - - $\quad$ AER thermal dissipations AEB 
Table 2

Parameter values associated with the path arrows shown in Fig. 4b. The values for $\left(\operatorname{Ra}_{c}, \operatorname{Re}_{c}\right)_{\mathrm{min}}$ denote the minimum, critical forcing rates needed for roll development, and the values for $\left(\operatorname{Ra}_{c}, R_{c}\right)_{s}$ up denote the supercritical forcing rates corresponding to each of the points $A, B_{1}, B_{2}$ and $B_{3}$ on the paths. Here $\mathrm{L}_{\mathrm{p}}, \mathrm{a}_{\mathrm{p}}, \beta_{\mathrm{p}}, \underset{\sim}{\mathrm{c}} \mathrm{p},\left|\omega_{\mathrm{p}}^{*}\right|$ and $\mathrm{T}_{\mathrm{p}}$ are the preferred values of horizontal wavelength, aspect ratio, orientation angle, propagation velocity, dimensionless frequency magnitude and period. The quantity $\mathrm{L}_{\mathrm{p}} / \mathrm{z}_{\mathrm{T}}$ represents the ratio of the horizontal to vertical roll scales, and $\overline{\mathrm{V}}$ is the average cross-roll wind velocity in the boundary layer. Other parameter values are: $\gamma_{\mathrm{d}}-\gamma_{\mathrm{e}}=0.1 \cdot \mathrm{C} / 600 \mathrm{~m}, \nu=\kappa=25 \mathrm{~m}^{2} / \mathrm{s}, \mathrm{P}=1, \mathrm{z}_{\mathrm{T}}=600 \mathrm{~m}, \mathrm{D}^{*}=1, \mathrm{q}=1$, $\mathrm{n}=2, \Lambda_{1}=-0.112, \Lambda_{2}=-0.094, \Lambda_{3}=-0.104, \Gamma_{1}=0.094, \Gamma_{2}=0.259$, and $\Gamma_{3}=0.242$.

\begin{tabular}{|c|c|c|c|c|c|c|c|c|c|}
\hline $\begin{array}{l}\text { Point } \\
\text { label }\end{array}$ & $\left(R a_{c}, R_{c}\right)_{\min }$ & $\left(\operatorname{Ra}_{c}, \operatorname{Re}_{c}\right)_{\text {s up }}$ & $\underset{(\mathbf{k m})}{\mathrm{L}_{\mathrm{p}}}\left(\mathrm{a}_{\mathrm{p}}\right)$ & $\beta_{\mathrm{p}}$ & $\begin{array}{c}\mathrm{c}_{\mathrm{p}} \\
(\mathrm{m} / \mathrm{s})\end{array}$ & $\left(\left|\omega_{p}^{*}\right|\right)$ & $\begin{array}{l}\mathrm{T}_{\mathrm{p}} \\
(\mathrm{hr})\end{array}$ & $\frac{L_{p}}{z_{T}}$ & $\begin{array}{c}\overline{0} \\
(\mathrm{~m} / \mathrm{s})\end{array}$ \\
\hline$A$ & $(5,60)$ & $(10,60)$ & $1.8(0.65)$ & $6^{\circ}$ & -0.3 & $(2.2)$ & 1.8 & 3.1 & -0.6 \\
\hline$B_{1}$ & $(10,30)$ & $(15,30)$ & $2.0(0.6)$ & $6^{\circ}$ & -0.2 & $(1.6)$ & 2.7 & 3.3 & -0.3 \\
\hline $\mathrm{B}_{2}$ & $(10,30)$ & $\left(25^{\downarrow}, 30\right)$ & $2.0(0.6)$ & $6^{\circ}$ & -0.2 & $(1.6)$ & 2.7 & 3.3 & -0.3 \\
\hline$B_{3}$ & $(10,30)$ & $(50,30)$ & $2.0(0.6)$ & $6^{\circ}$ & -0.2 & $(1.6)$ & 2.7 & 3.3 & -0.3 \\
\hline
\end{tabular}


Table A1

Coefficient Definitions for the Spectral Model (A1) - (A14).

\begin{tabular}{|c|c|c|c|c|}
\hline$i$ & $a_{i}$ & $b_{i}$ & $c_{i}$ & $\mathrm{~d}_{\mathrm{i}}$ \\
\hline 1 & $\frac{1}{2} \frac{(n-q)}{\left(a^{2}+q^{2}\right)}\left[q^{2}-2 q n-a^{2}\right]$ & $\frac{p}{\left(a^{2}+q^{2}\right)}$ & $\frac{P}{\left(a^{2}+q^{2}\right)}\left[\left(a^{2}+q^{2}\right) \Lambda_{1}+\Gamma_{1}\right]$ & $\frac{\left(a^{2}+q^{2}\right)}{a}$ \\
\hline 2 & $\frac{1}{2} \frac{(n+q)}{\left(a^{2}+q^{2}\right)}\left[a^{2}-2 q n-q^{2}\right]$ & $\frac{P}{\left(a^{2}+n^{2}\right)}$ & $\frac{p}{\left(a^{2}+q^{2}\right)}\left[\left(a^{2}+n^{2}\right) \Lambda_{2}+\Gamma_{2}\right]$ & $\frac{\left(a^{2}+n^{2}\right)}{a}$ \\
\hline 3 & $\frac{1}{2} \frac{(n-q)}{\left(a^{2}+n^{2}\right)}\left[n^{2}-2 q n-a^{2}\right]$ & $\ldots$ & $\frac{p}{\left(a^{2}+n^{2}\right)}\left[\left(a^{2}+n^{2}\right) \Lambda_{3}+\Gamma_{3}\right]$ & $\frac{(n-q)^{2}}{a}$ \\
\hline 4 & $\frac{1}{2} \frac{(n+q)}{\left(a^{2}+n^{2}\right)}\left[a^{2}-2 q n-n^{2}\right]$ & $-\cdots$ & $\frac{p}{\left(a^{2}+n^{2}\right)}\left[\left(a^{2}+q^{2}\right) \Lambda_{2}+\Gamma_{2}\right]$ & $\frac{(n+q)^{2}}{a}$ \\
\hline 5 & $\frac{1}{2}(n+q)$ & $-\ldots$ & $P \Lambda_{1}$ & $\frac{4 q^{2}}{a}$ \\
\hline 6 & $\frac{1}{2}(n-q)$ & $\cdots$ & $P \Lambda_{2}$ & $\frac{4 n^{2}}{a}$ \\
\hline 7 & $q$ & - . - & $\mathrm{P} \Lambda_{3}$ & $\ldots$ \\
\hline 8 & $\mathrm{n}$ & $\ldots$ & -.... & $\ldots$ \\
\hline
\end{tabular}

Table A2

Definitions of the Fourier Coefficients $\Lambda_{i}$ and $\Gamma_{i}$.

\begin{tabular}{|c|c|c|}
\hline $\mathbf{i}$ & $\Lambda_{i}$ & $\Gamma_{i}$ \\
\hline 1 & $\frac{2}{\pi} \int_{0}^{\pi} 0^{*} \sin ^{2}\left(q z^{*}\right) d z^{*}$ & $\frac{2}{\pi} \int_{0}^{\pi} \frac{\partial^{2} \sigma^{*}}{\partial z^{* 2}} \sin ^{2}\left(q z^{*}\right) d z^{*}$ \\
\hline 2 & $\frac{2}{\pi} \int_{0}^{\pi} U^{*} \sin \left(q z^{*}\right) \sin \left(n z^{*}\right) d z^{*}$ & $\frac{2}{\pi} \int_{0}^{\pi} \frac{\partial^{2} \sigma^{*}}{\partial z^{* 2}} \sin \left(q z^{*}\right) \sin \left(n z^{*}\right) d z^{*}$ \\
\hline 3 & $\frac{2}{\pi} \int_{0}^{\pi} \sigma^{*} \sin ^{2}\left(n z^{*}\right) d z^{*}$ & $\frac{2}{\pi} \int_{0}^{\pi} \frac{\partial^{2}{U^{*}}^{*}}{\partial z^{* 2}} \sin ^{2}\left(n z^{*}\right) d z^{*}$ \\
\hline
\end{tabular}




\section{RADIATIVELY-DRIVEN INTERACTIONS BETWEEN STRATOCUMULUS AND SYNOPTIC WAVES}

In a paper accepted for publication in the Journal of the Atmospheric Sciences (Clark, 1993), the effect of radiative cooling perturbations above stratocumulus on the structure of synoptic-scale waves is considered. The cloud position is linked to the waves via the phase of the low-level streamfunction. Coupling is strongest with cloud to the west of surface troughs. The resulting stationary structures capture the summertime pattern of stratocumulus off California and its linkage to the mid-Pacific ridge. The implication is that cloud-induced radiative cooling above cloudy regions plays an important role in determining observed structures over marine areas.

A copy of this paper is attached. 


\title{
RADIATIVELY-DRIVEN INTERACTIONS
}

BETWEEN STRATOCUMULUS

AND SYNOPTIC WAVES

\author{
John H.E. Clark \\ Department of Meteorology \\ The Pennsylvania State University \\ University Park, Pennsylvania 16802
}

Submitted to Journal of the Atmospheric Sciences 


\section{ABSTRACT}

Quasi-geostrophic disturbances on a mid-latitude $\beta$-plane channel forced by radiative heating perturbations due to stratocumulus are considered. Longitudinal phase of the cloud is fixed to that of the lowlevel stream function. The background flow has a jet centered near the tropopause. Cloudiness is wavelike with cooling above cloudy areas that decreases exponentially with height. No perturbation cooling occurs above cloud-free areas. Forced steady waves are found. Though infinitesimal amplitude disturbances are considered, the problem is nonlinear because of the coupling between cloud and winds. The resulting structures are sensitive to the phase relationship between cloud and stream function with strongest coupling for cloud to the west of surface troughs. The waves have vertical scales roughly the troposphere depth. The stationary structures capture the summertime pattern of stratocumulus off California and its linkage to the midPacific ridge. Zonal mean cloud cooling forces an adjustment to the mean westerlies that strengthens them in the northern half of the channel near the lower boundary. When this correction is allowed for, synoptic-scale amplitudes increase (decrease) just above the cloud in the northern (southern) part of the channel. Mean cloud cooling also renders the background potential vorticity gradient negative and thus baroclinically unstable just above the cloud in the northern domain. 


\section{Introduction}

Interactions between clouds and winds have important, but poorly understood, influences on atmospheric variability. Daily weather changes, as well as secular variations on inter-annual and climatic time scales, are modulated by such interactions. On the climatic scale, a major uncertainty in assessing the susceptibility of the general circulation to increasing $\mathrm{CO}_{2}$ concentrations is the role of clouds. Lindzen (1990) doubts, because of the primitive state of our understanding of cloud-mediated feedbacks, whether it can be stated with certainty that global temperatures will increase in response to increasing $\mathrm{CO}_{2}$. Randall et al. (1984) show that cooling due to a $4 \%$ increase in global coverage by low-level stratiform cloud could more than offset the widely-predicted $2-4{ }^{\circ} \mathrm{K}$ rise in global temperatures due to a doubling of $\mathrm{CO}_{2}$.

There has been a lack of studies whose aim is to understand the interaction between motion systems and stratocumulus cloud. This is unfortunate because of widespread coverage by this cloud. For instance, according to Campana (1988), the largest contributor to total cloud cover at mid- and high latitudes is low cloud followed by middle and then high cloud. Also, Rossow and Schiffer (1991) show that in either hemisphere from $20^{\circ}$ to $50^{\circ}$ latitude most cloud tops occur at pressures of $800 \mathrm{mb}$ or greater.

Since the solar albedo of stratocumulus (typically 0.4 ) exceeds that of the underlying surface (typically $<0.1$ for water and 0.2 for land), there is a deficit of absorbed solar radiation at the surface in cloudy regions. Near the ground, stratiform clouds also warm by absorbing and emitting infrared radiation. Ramanathan et al. (1989) show, however, 
that the net radiative effect within the cloud and below is cooling.

Thick decks of stratiform cloud also modify upwelling infrared radiation above the cloud. They emit less radiation than the lower boundary (especially over land areas) since their emission temperature is less than that of the underlying surface. The only exception is at high latitudes where cloud top temperatures can be higher than lower boundary temperatures and stratiform clouds can warm the atmospheric column above them. Randall et al. (1984) and Sohn and Smith (1992) show up to a $10 \%$ or $40 \mathrm{~W} \mathrm{~m}^{-2}$ decrease in satellitemeasured upwelling infrared radiances due to marine stratocumulus off the coast of California.

Donner and Kuo (1984) calculated infrared heating rates allowing for low-level stratiform clouds and find, at mid-latitudes, a perturbation due to clouds of about $2 \mathrm{~K} \mathrm{~d}^{-1}$ near cloud top. They also find stationary quasi-geostrophic structures forced by topography, surface latent and sensible heating, and radiative heating (as modified by clouds). Resulting wave structures closely agree with tropospheric observations. Cloud induced asymmetries, they find, account for at least $20 \%$ of the calculated wave amplitudes. Donner and Kuo neglected meridional gradients of the mean zonal wind thus precluding wave energy accumulation in certain latitudinal belts because of meridional variations in refractive index (Karoly and Hoskins; 1982). This could lead to a significant error in their estimate of synoptic-scale sensitivity to cloud heating. There have been many other studies of the response of large-scale waves to stationary patterns of heating (Smagorinsky, 1953; Derome and Wiin Nielsen, 1971; Ashe, 1979; Hoskins and Karoly, 1981; Lindzen and Jacqumin, 1982; Alpert et al., 1983). In these studies, clouds, if accounted for, are geographically fixed and not linked in any way to the disturbances they modify. 
This study focuses on the effects of perturbations to the field of radiative heating due to stratiform clouds. The calculations are dynamical in that the cloud location is tied to the synoptic-scale disturbance it forces. Cooling rates due to stratocumulus will be very modest (a few deg. $\mathrm{K} \mathrm{d}^{-1}$ ). Calculated radiative cooling rates in very thin layers near the top of widespread cloud decks are roughly an order of magnitude larger (Nicholls, 1984). When this cooling spreads over a horizontal layer whose thickness matches the vertical scale of typical synoptic-scale disturbances, it is roughly of the magnitude used.

Certain stationary features of the large-scale cloud distribution link to synoptic scale wind patterns. For instance, persistent decks of marine stratocumulus occur in regions of subsidence to the east of subtropical highs at mid-latitudes (Schubert, 1976; Schubert et al., 1979; Warren et al.,1988). In the northern hemisphere, this stratocumulus is most extensive during the summer when subsidence associated with the subtropical highs is strongest (Schubert et al., 1979). In these regions, a strong temperature inversion with warm, dry air aloft caps the stratocumulus. Turbulence generated by shear in the cool moist air below the inversion and cloud top radiative cooling combine to maintain a sharp elevated base to the inversion layer (Lilly, 1968; Schubert, 1976). Schubert et al. (1979) show that for a horizontally homogeneous steady-state cloud deck, the strength of the synoptic-scale divergence affects both cloud base and cloud top height: the stronger the divergence, the lower the cloud base and top and the thinner the cloud. Both sensible and latent heat flux divergences thus will be modified by divergence and thus there could be important feedbacks on the synoptic scale via this mechanism. Thus feedbacks of stratocumulus on synoptic scale motions are complex and are not solely due to low-level radiative cooling. This study concentrates on radiatively-driven coupling. 
Other features of the stratiform cloud distribution closely couple to traveling weather systems. For example, the movement of cold air masses off the east coast of Asia and North America during winter behind propagating cyclones often leads to widespread marine stratocumulus. Also warm-frontal overrunning often creates broad regions of cloud ahead of propagating cyclones.

It is thus reasonable to link the longitudinal phase of some features of the observed stratocumulus distribution to the phase of synopticscale wind and thermal patterns. The focus of this study is to examine the structure of synoptic-scale features that result from this coupling using linear quasi-geostrophic theory applied to a mid-latitude $\beta$-plane. These structures will be compared with observations over marine regions where the effects of inhomogeneities in the underlying lower boundary are minimal.

Assume the cloud distribution along a latitude circle is wavelike. Since cooling normally occurs in and above cloudy regions, mean and wavy components of cooling result, see Fig. 1 . The mean cooling, if a function of latitude, can change the zonally-averaged winds and temperatures. They, in turn, can feed back on the synoptic-scale waves that couple with low-level clouds. This study will separately consider structures driven by mean and wavy components of the cloud cooling as well as feedbacks on stationary waves triggered by mean cloud cooling.

Theory appears in Section 2 and results presented for an analytical constant background wind model. Stationary wave structures with a realistic background atmosphere are presented in Section 3. Forcing is by a combination of fixed lower boundary heating (to simulate geographically fixed patterns due to land-sea contrast) and cloud. 


\section{Theory}

The linearized quasi-geostrophic potential vorticity equation is

$$
\frac{\partial q^{\prime}}{\partial t}+U \frac{\partial q^{\prime}}{\partial x}+\frac{\partial Q}{\partial y} \frac{\partial \psi^{\prime}}{\partial x}=\frac{f_{0}}{\rho_{0}} \frac{\partial}{\partial z}\left(\frac{\rho_{0} h^{\prime}}{N^{2}}\right)-d q^{\prime},
$$

where $\psi^{\prime}$ is the stream function. The vertical coordinate is log pressure, $z=H \ln \left(p_{0} / p\right): H$ is scale height, $p_{0}=1000 \mathrm{mb}$. The geostrophic winds $u_{g}$ and $v_{g}$ are, respectively, $-\partial \psi^{\prime} / \partial y$ and $\partial \psi^{\prime} / \partial x$. On a $\beta$-plane, the Coriolis parameter $f=f_{0}+\beta y$. The background density $\left(\rho_{0}\right)$ is a function of $z$ only. Linear dissipation in (2.1) arises from Newtonian cooling and Rayleigh friction. For convenience, the time scale of each is $T_{d}=d^{-1}$. The perturbation potential vorticity, q', relates to $\psi^{\prime}$ by

$$
q^{\prime}=\nabla^{2} \psi^{\prime}+\frac{f_{0}^{2}}{\rho_{0}} \frac{\partial}{\partial z}\left(\frac{\rho_{0}}{N^{2}} \frac{\partial \psi^{\prime}}{\partial z}\right) .
$$

Define the heating rate, $h^{\prime}$ (dimension $\mathrm{m} \mathrm{s}^{-3}$ ), from the thermodynamic equation:

$$
\frac{\partial b^{\prime}}{\partial t}+U^{\partial b^{\prime}} \frac{\partial B}{\partial x}+\frac{\partial \psi^{\prime}}{\partial y}+N^{2} w^{\prime}=h^{\prime}-d b^{\prime},
$$


where $b^{\prime}$ and $B$ are, respectively, perturbation and background buoyancies. The buoyancy frequency squared is $N^{2}=d B / d z$. The hydrostatic and thermal wind equations are, respectively,

$$
b^{\prime}=f_{0} \frac{\partial \psi^{\prime}}{\partial z},
$$

and

$$
\frac{\partial U}{\partial z}=-\frac{1}{f_{0}} \frac{\partial B}{\partial y}
$$

The background potential vorticity gradient is

$$
\frac{\partial Q}{\partial y}=\beta-\frac{\partial^{2} U}{\partial y^{2}}+\frac{f_{0}^{2}}{\rho_{0}} \frac{\partial}{\partial z}\left(\frac{\rho_{0}}{N^{2}} \frac{\partial U}{\partial z}\right) .
$$

For steady heating at a single wavenumber $\mathrm{k}$ in the $\mathrm{x}$ direction, and (2.2) combine to yield a diagnostic equation for $\psi^{\prime}$ :

$$
\frac{\partial^{2} \Psi}{\partial y^{2}}+\frac{f_{0}^{2}}{\rho_{0}} \frac{\partial}{\partial z}\left(\frac{\rho_{0}}{N^{2}} \frac{\partial \Psi}{\partial z}\right)+\left(\frac{1}{U^{*}} \frac{\partial Q}{\partial y}-k^{2}\right) \Psi=-\frac{i f_{0}}{k U^{*} \rho_{0}} \frac{\partial}{\partial z}\left(\frac{\rho_{0} \Pi}{N^{2}}\right),
$$

where $\psi^{\prime}$ and $h^{\prime}$ are, respectively, $\Psi(y, z) \exp (i k x)$ and $\Pi(y, z) \exp (i k x)$. Dissipation is accounted for by letting

$$
U^{*}=U(y, z)-i /\left(k T_{d}\right)
$$

Equation (2.7) is of the Helmholtz type and can usually be solved by successive over-relaxation. The local nature of its solution, neglecting dissipation for the moment, depends on the factor 


$$
R \equiv \frac{1}{U} \frac{\partial Q}{\partial y}-k^{2}
$$

In regions of the $y-z$ plane where $R$ is positive, a stationary solution is wavelike. This implies wave energy propagation. Where $R$ is negative, energy propagation is not possible.

a. Constant background wind

Consider the response to heating where the wave and associated cloud have an arbitrary zonal phase speed $c$. Waves that neither grow nor decay with time, in spite of the radiative forcing, are considered. The wavy component of cloud heating is

$$
h^{\prime}=\Pi \exp \left[i k(x-c t)+i \ell y-\frac{z}{D}\right] .
$$

The amplitude $\Pi$ is complex with modulus $2 \mathrm{~K} \mathrm{~d}^{-1}$ times $\mathrm{g} / \theta_{\mathrm{o}}$ ( $\mathrm{g}$ is gravity and $\left.\theta_{0}=273 \mathrm{~K}\right)$. Heating decays exponentially with height with e-folding depth $D=10 \mathrm{~km}$ but without any phase change in keeping with the model in Fig. 1. In this section and those to follow, only the region above cloud top, located at $z=0$, is resolved. The lower boundary condition of zero synoptic-scale vertical velocity is also applied at $z=0$.

If the background wind is constant, $\partial Q / \partial y=\beta$ in (2.6); becomes 


$$
(U-c) \frac{\partial q^{\prime}}{\partial x}+\beta \frac{\partial \psi^{\prime}}{\partial x}+d q^{\prime}=\frac{f_{0}}{N^{2}} \frac{\partial h^{\prime}}{\partial z}
$$

where $\mathrm{N}^{2}$ is assumed constant. If the perturbation stream function, $\psi^{\prime}$, varies with height as $\exp (\mathrm{imz})$, the perturbation potential vorticity becomes $q^{\prime}=-\left(k^{2}+\ell^{2}\right) \psi^{\prime}-f_{0}^{2}\left(m^{2}+i m / H\right) \psi^{\prime} / N^{2}$.

In (2.10), $\psi^{\prime}$ is the sum of a particular solution to the full equation (with heating) and a general solution without heating. If $\psi^{\prime}$ has the horizontal structure $\exp (i k x+i \ell y)$, the homogeneous solution is $B$ $\exp [i(k x+\ell y+m z)]$ provided

$$
m^{2}+\frac{i m}{H}=\frac{N^{2}}{f_{0}^{2}}\left(\frac{\beta}{U^{*}-c}-k^{2}-\ell^{2}\right) .
$$

The constant $B$ is not yet known.

The particular solution is $A \exp [i k(x-c t)+i \ell y-z / D]$ provided, using (2.11),

$$
A=-\frac{\Pi}{i k f_{0} D U *\left(m^{2}+\frac{i m}{H}+\frac{1}{D^{2}}+\frac{1}{D H}\right)}
$$

The solution to $(2.10)$ is now

$$
\left.\psi^{\prime}=\exp [i k(x-c t)+i \ell y] \mid A \exp \left(-\frac{z}{D}\right)+B \exp (i m z)\right\}
$$

The constant $B$ follows from the thermodynamic equation applied at the lower boundary. Because of the log pressure vertical coordinate, the 
boundary condition of zero material vertical velocity must be applied with caution. The Appendix shows that a new non-Doppler term appears in the thermodynamic equation at $z=0$ for transient waves. It differs from the non-Doppler term discussed in Lindzen (1968) and Geisler and Dickinson (1975). They keep two terms arising from the expansion of material vertical velocity that cancel and thus obtain a different, but equivalent, formulation. The boundary condition at $z=0$ for a constant mean wind is

$$
f_{0}\left(\frac{\partial}{\partial t}+U \frac{\partial}{\partial x}\right) \frac{\partial \psi^{\prime}}{\partial z}-\frac{f_{0} N^{2}}{g} \frac{\partial \psi^{\prime}}{\partial t}+f_{0} d \frac{\partial \psi^{\prime}}{\partial z}=h^{\prime}
$$

The non-Doppler term is the second on the left side.

The constant $B$ follows upon substituting (2.12) and (2.13) into (2.14):

$$
B=-\frac{i \Pi\left(m^{2}+\frac{i m}{H}+\frac{1}{D H}+\frac{N^{2} c}{g D U^{*}}\right\}}{\left.k\left\{m^{2}+\frac{i m}{H}+\frac{1}{D^{2}}+\frac{1}{D H}\right) \mid i m f_{0} U^{*}+\frac{f_{0} N^{2} c}{g}\right\}} .
$$

The stream function amplitude at the lower boundary is, using (2.12) and (2.15),

$$
A+B=-\frac{i \Pi\left(m^{2}+\frac{i m}{H}-\frac{i m}{D}+\frac{1}{D H}\right)}{k f_{0}\left(m^{2}+\frac{i m}{H}+\frac{1}{D^{2}}+\frac{1}{D H}\right)\left(\frac{N^{2} c}{g}+i m U^{*}\right)}=\Pi G .
$$

The last equality defines $G$. Equation (2.16) enables the phase of the cloud field at $z=0$ relative to that of the stream function to be found as a function of $U, k, d$ (or $T_{d}$ ),,$H$, and $D$. Since the cloud is $180^{\circ}$ out of phase with the heating (see Fig. 1), (2.16) states that phase\{stream 
function $\}-$ phase $\{$ cloud $\}=\arg \{-G\}$. Suppose, for instance, $\arg \{-G\}=-$ $90^{\circ}$. Cloud would be concentrated east of troughs and, if they tilt westward with height, on their warm side. $\operatorname{Arg}\{-G\}=+90^{\circ}$ implies cloud to the west of surface troughs. Fig. $2 a$ shows the sensitivity of $\arg \{-G\}$ to zonal wavenumber and background wind for stationary waves $(c=0)$ with $T_{d}=10 \mathrm{~d}, D=H=10 \mathrm{~km}$ and $\mathrm{N}=0.01 \mathrm{~s}^{-1}$. This value of $T_{d}$ is in accord with the detailed calculations of Prinn (1977) for the radiative time scale of disturbances above an insulated lower boundary with a vertical wavelength about twice the troposphere depth. With westerly background flow, there is a broad region, especially for large wavenumbers, where the cloud mainly occurs to the east of the surface trough. Background easterlies or weak westerlies (especially for large east-west wavelengths or small zonal wavenumbers) favor cloud organized to the west of surface troughs.

Wave stationarity has eliminated the non-Doppler term in (2.14). However, calculations show that even with traveling waves, this term is very small. Thus all solutions are mainly dependent on the Dopplershifted background wind, $U-c$, and not independently on $U$ and $c$. Therefore the ordinates in Figs. $2 a$ and $2 b$ could, without significant loss in accuracy, be U-c.

The dimensionless surface stream function amplitude in Fig. $2 b$ follows from (2.16) after dividing by $g^{2} /\left(f_{0} N^{2}\right)$. Background Dopplershifted westerlies and small zonal wavenumbers favor the largest response to stratocumulus cooling. One locus of maximum amplitude, labeled critical, occurs for U-c $=0$.

A second sloping locus of maximum response, labeled external Rossby, occurs in the region of westerly Doppler-shifted background winds. For zonal wavenumber five, according to Fig. 3 , the vertical 
wavelength, $2 \pi / R e(m)$, near this locus is over $100 \mathrm{~km}$. The imaginary part of $m$, from Fig. 3 , gives a $28 \mathrm{~km}$ e-folding depth for amplitude decay. Thus the mode is almost barotropic. If it was not for dissipation, the mean flow of about $8 \mathrm{~m} \mathrm{~s}^{-1}$ corresponding to this mode would render it as trapped or evanescent. Weak dissipation forces the wave to exhibit a slight westward tilt with height at all wavelengths and background winds. Stratocumulus primarily lies to the east of surface troughs for the external Rossby mode. Phase of the cloud field relative to that of the surface stream function is very sensitive to the Dopplershifted background wind as witnessed by the concentration of constant phase lines near this locus in Fig. 4a.

Marine stratocumulus off California lies to the east of the subtropical Pacific high in a region of weak westerlies. The pattern roughly corresponds to zonal wavenumber three to five and roughly conforms to the stationary critical mode structure found above. According to the theory, traveling stratocumulus patterns that radiatively couple to synoptic waves would take on the external Rossby mode structure with cloud concentrated to the east of surface troughs. Unfortunately there have not been enough observational studies of cyclonic systems to confirm or deny this linkage.

\section{b. Synoptic-scale vertical motion}

Summertime marine stratocumulus off the west coast of North America occurs in a region of synoptic-scale subsidence. The relationship between cloud location (as determined by the pattern of radiative cooling) and lower troposphere synoptic-scale vertical motion is implicit with the present analytical model. If the cloud coincides with descent, then just above the cloud radiational cooling is offset somewhat by compressional heating. Also, the capping inversion 
should be stronger in regions of synoptic-scale subsidence. Alternatively, if rising motion occurs in the region of low-level cloudiness, adiabatic cooling supplements cloud cooling.

Substitute (2.14) into (2.3). The result is

$$
N^{2} w^{\prime}=\left[\Pi e^{-z / D}-i k f_{0} U^{*}\left(-\frac{A}{D} e^{-z / D}+i m B e^{i m z}\right)\right] e^{i k(x-c t)+i \ell y} .
$$

After (2.12) and (2.15) are used for $A$ and $B$, respectively, and (2.11) solved for $m, w^{\prime}$ can be found for a given heating rate amplitude $\Pi$. Write the result of these substitutions into (2.17) as $N^{2} W^{\prime}=R h^{\prime}$, where $R$ is complex and $h^{\prime}=\Pi \exp [i k(x-c t)+i \ell y]$. The sum of cloud radiative and compressional heating is thus $h^{\prime}-N^{2} w^{\prime}=(1-R) h^{\prime}$. Thus $1-R$ is the ratio of total heating to cloud radiative heating.

The phase of $1-R$ at $1 \mathrm{~km}$ for stationary waves appears in Fig. 4 as a function of background wind and zonal wavenumber. For the reasons stated above, the background flow can, with little loss in accuracy, be considered the Doppler-shifted flow $(U-c)$. In the shaded region, rising motion mainly occurs above cloudy regions that cool radiatively. Wavenumbers less than about six exhibit this anomalous behavior provided $U-c<-5 \mathrm{~m} \mathrm{~s}^{-1}$. For U-c greater than about $-5 \mathrm{~m} \mathrm{~s}^{-1}$ and/or wavenumbers greater than about seven, low-level subsidence occurs predominantly in cloudy regions that cool radiatively.

The latter pattern is most commonly observed in regions of persistent stratocumulus such as off the west coast of North America. Subsidence reinforces the capping inversion in these regions leading to a more permanent cloud deck. Again this simple theory agrees fairly well with observations of marine stratocumulus. 


\section{Influence of Background Wind Shear}

a. Response to wavy heating

Consider steady waves forced by wavy heating on a mid-latitude $\beta$ plane channel with a realistic background flow:

$$
U(y, z)=U_{0}+U_{s} \cos (\ell y) \exp \left[-\left(z-z_{0}\right)^{2} / D_{U}^{2}\right]
$$

with $U_{0}=5 \mathrm{~m} \mathrm{~s}^{-1}, U_{S}=15 \mathrm{~m} \mathrm{~s}^{-1}, z_{0}=10 \mathrm{~km}, D_{U}=10 \mathrm{~km}$ for $z<z_{0}$ and $D_{U}=20 \mathrm{~km}$ for $z>z_{0}, \ell=\pi / 3500 \mathrm{~km}^{-1}$. A $20 \mathrm{~m} \mathrm{~s}^{-1}$ jet, Fig. $5 a$, centered at the tropopause and mid-channel, $y=0$, is described. The associated potential vorticity gradient, $\partial Q / \partial y$, in Fig. $5 b$ is positive everywhere with a maximum $2 \mathrm{~km}$ below the jet core. A stability jump occurs at the tropopause such that $N=10^{-2} \mathrm{~s}^{-1}\left(2.5 \times 10^{-2} \mathrm{~s}^{-1}\right)$ for $z<z_{0}\left(z>z_{0}\right)$.

The wavy heating has two components.

- Fixed Pattern due to Land-Sea Temperature Contrast

It is proportional to $\cos (k x) \cos (\ell y)$ with amplitude ${ }^{1} 2 \mathrm{~K} \mathrm{~d}^{-1}$ times $g / \theta_{0}$ and occurs at the lower boundary. This heating is introduced by the thermodynamics Eq. (2.3) applied at $z=0$. Discretized Eq. (2.7) is solved in the interior on a $0.5 \times 175 \mathrm{~km}$ height-latitude. The fixed heating does not contribute to the forcing term in (2.7) at any interior location. Thus this heating has a Dirac delta function

1 An amplitude of $2 \mathrm{~K} \mathrm{~d}^{-1}$ for the wavy component is equivalent to a cooling rate of $\pi / 2$ times $2 \mathrm{~K} \mathrm{~d}^{-1}$ or $3.14 \mathrm{~K} \mathrm{~d}^{-1}$ in the clouc; areas and zero cooling in cloud free areas according to Fourier's theorem. 
distribution, $\delta(z)$, in the vertical.

- Variable Phase Component due to Stratocumulus

It links to the stream function at the lower boundary by an arbitrary phase $\Phi_{c}$; if the stream function varies as $\cos (k x)$, the cloud cover varies as $\cos \left(k x-\Phi_{C}\right)$. Negative cloud, of course, does not exist. Since cloud is $180^{\circ}$ out of phase with radiative heating, see Fig. 1, $\Phi_{C}=$ $90^{\circ}\left(-90^{\circ}\right)$ signifies that stratocumulus coincides with low-level cold (warm) air advection to the west (east) of surface troughs assuming they tilt westward with increasing height. Thus $\Phi_{C}$ equals $\arg \{-G\}$ discussed in Section 2a. Heating decays as $\exp (-z / D)$ where $D=10 \mathrm{~km}$. Its formal representation is $h^{\prime}=\Pi \exp (i k x)$ where

$$
\Pi=\Pi_{0} \frac{\psi^{\prime}(z=0)}{\left|\psi^{\prime}(z=0)\right|} \exp \left(-i \Phi_{c}-\frac{z}{D}\right) \cos (l y)
$$

At mid-channel $y=0$. Also $\Pi_{0}=2 \mathrm{Kd}^{-1}$ times $\mathrm{g} / \theta_{0}$.

Substitute (3.2) into (2.7); the steady response to steady wavy heating follows. Impose a radiation condition at the domain top (20 $\mathrm{km}$ ) and set the stream function equal to zero at the northern and southern walls, which are $W=3500 \mathrm{~km}$ apart.

There are some important points to note.

- The fixed component of wavy heating is indispensable since it creates the disturbance to which the cloud and its cooling pattern is attached. That disturbance, in turn, is modulated in amplitude and phase by the cloud. 
- Although (2.7) is linear, condition (3.2) linking the cloud cooling and stream function renders the problem nonlinear. Sequential relaxation, nevertheless, was used to solve (2.7). Convergence, when achieved, was slow because cloud cooling was repeatedly adjusted according to (3.2) at each latitude. The algorithm succeeded for zonal wavenumbers greater than three. Planetary-scale solutions (wavenumbers one, two and three) were not found since these wavelengths are propagating (as will be shown later) at almost all channel locations.

- In contrast to the constant background wind model, only the forced response to heating was found. It was not supplemented by a solution of the homogeneous problem.

Wavy heating could trigger instabilities of the background state. Since, the potential vorticity gradient, $\partial Q / \partial y$, is positive everywhere, internal baroclinic instability (Charney and Stern, 1962) is precluded. Vertical shear near $z=0$ is equivalent to a sheet close to the boundary where the potential vorticity increases to the south, Bretherton (1966). External baroclinic instabilities (James and Hoskins, 1985) are thus possible. They would have largest amplitude near the lower boundary and thus could have important implications on cloud-induced feedbacks. This study focuses on the steady response to heating and ignores spontaneous background instabilities.

WKB theory is helpful in understanding solutions of (2.7). Karoly and Hoskins (1982) apply ray theory to interpret planetary wave propagation in regions of slowly (for WKB theory to be valid) varying $R$, defined in (2.9). Assume $R$ is positive. Wave energy propagates along a ray or characteristic with a speed equal to the local group velocity. Rays tend to be parallel to the vector gradient of $R$. Wave energy thus 
focuses toward regions of maximum $R$. Zero wind lines where $U=0$ and thus $R$ is infinite will be locations of energy accumulation by stationary waves. As energy approaches the zero wind line, the group velocity approaches zero and the energy takes an infinite time to get there (at least according to linear, inviscid theory).

Note, first, that locally unforced $(\Pi=0)$ solutions to (2.7) exist. In the inviscid WKB limit, the condition

$$
\kappa^{2}=\frac{1}{U} \frac{\partial Q}{\partial y}-\frac{f_{0}^{2}}{N^{2}}\left(\mu^{2}+\frac{1}{4 H^{2}}\right)
$$

must be satisfied, where $\mathrm{k}$ is the horizontal wavenumber, $\kappa^{2}=k^{2}+\ell^{2}$, and

$$
\mu=m+\frac{i}{2 H} .
$$

The stream function amplitude is wavelike in $y$ and $z: \Psi \propto \exp (i \ell y+i m z)$. The vertical wavenumber $m$ can be complex, but $\mu^{2}$ is real. For $\mu^{2}>0, \Psi$ amplifies with height as $\exp (z / 2 H)$. An unforced solution is locally propagating (evanescent) if $\mu^{2}>0\left(\mu^{2}<0\right)$.

Equation (3.3) relates the horizontal and vertical wavenumbers of a stationary free wave. Let $k_{c r}$ be the zonal wavenumber at mid-channel (latitude $45^{\circ} \mathrm{N}$ ) where the transition between evanescent $\left(k<k_{c r}\right)$ and vertically propagating $\left(k>k_{c r}\right)$ behavior occurs. Set $\mu$ equal to zero in (3.3); then

$$
k_{c r}^{2}=\frac{1}{U} \frac{\partial Q}{\partial y}-\frac{f_{0}^{2}}{4 N^{2} H^{2}}-\ell^{2}
$$


Fig. 6 shows the integral zonal wavenumber $n_{c r}=r k_{c r} \cos \left(45^{\circ}\right)$ for north-south wavelengths: $\lambda_{y}=W$ and $2 W\left(\lambda_{y}=2 \pi / \ell\right)$. The earth's radius is $r$. Mid-channel values of $U$ and $\partial Q / \partial y$ are used. The jump in $n_{c r}$ across the tropopause reflects the sudden change in $N$. Ultra-long waves with wavenumbers one through four are propagating in most of the troposphere. The exception is very close to the ground where long-wave trapping occurs for $\lambda_{y}=W$ due to a maximum in curvature of $U$ with respect to $z$. Short wave trapping for wavenumbers greater than six occurs for all $z$. All wavenumbers (except one) become or are trapped in the stratosphere. Turning points for longer wavelengths develop at larger altitudes than for shorter wavelengths.

Consider a wave forced by stationary wavy heating, $\Pi \exp (i k x)$, at $z=0$. Neglect dissipation. Use (2.5) for $\partial B / \partial y$ and apply (2.3) at $z=0$ where $w=0$ :

$$
i k f_{0}\left(i m U-\frac{\partial U}{\partial z}\right) \Psi=\Pi
$$

Since the wave is stationary, $w=0$ is equivalent to setting the material vertical velocity equal to zero at the lower boundary. A resonant response to heating occurs if the bracketed term on the left side of (3.6) is zero.

The mid-channel amplitude and phase of $1 /(\mathrm{imU}-\partial U / \partial \mathrm{z})$ appear in Fig. 7 for $\lambda_{y}=2 W=7000 \mathrm{~km}$. The vertical wavenumber, $m$, follows from (2.11); acceptable roots have $\operatorname{Im}(\mu)>0$ thus preventing growth with $z$ faster than $\exp (z / 2 H)$. The amplitude in Fig. 7 exhibits a broad peak near wavenumber five. In the absence of dissipation, the zonal wavenumber for a resonant response to the wavy heating is 5.35 . The phase in Fig. 7 undergoes a shift of 100 to $110^{\circ}$ near the peak response. Held (1983) shows that the resonant response is an external Rossby 
wave. With no dissipation, the resonant wave grows with $z$ as $\exp \left(z \mathrm{U}^{-}\right.$ $1 \partial U / \partial z$ ) near the lower boundary. In the mid-and upper troposphere, according to Fig. 6, the quasi-resonant response (wavenumber five) is propagating. A turning point occurs at the tropopause and the response is trapped in the stratosphere. These findings are consistent with those of Held (1983).

Fig. 8 compares normalized amplitudes zonal wavenumber five for $\Phi_{C}=-90^{\circ}$ and $+90^{\circ}$. Wave structures are in accord with deductions concerning locally evanescent and propagating behavior that follow from Fig. 6. Both structures grow in amplitude from $z=0$ to a maximum near the westerly jet core (mainly due to the decrease of background density). Wave evanescence forces decay in the stratospheric westerlies. The only significant difference between Fig. $8 a$ and $8 b$ occurs near domain base at mid-channel. With cloud to the west of surface troughs $\left(\Phi_{C}=90^{\circ}\right)$, wavenumber five here is almost equal in amplitude to the jet core maximum. The $\Phi_{C}=-90^{\circ}$ structure is much more sharply peaked near the jet maximum; amplitudes near domain base at mid-channel are smaller than for $\Phi_{C}=90^{\circ}$. The $\Phi_{C}=90^{\circ}$ lower troposphere structure is amplified by cloud radiative cooling since cold air lying to the west of surface troughs (the waves tilt westward with increasing height) is cooled radiatively. The $\Phi_{C}=-90^{\circ}$ structure is weakened by radiative cooling since warm air located immediately above cloudy areas east of surface troughs is cooled.

The Eliassen-Palm (EP) flux, shown in Fig. 9 for $\Phi_{C}=-90^{\circ}$, is a useful diagnostic of quasi-geostrophic disturbances (Edmon et al. ,1980). Not only does the EP flux permit an evaluation of wave-mean flow interactions, it serves as a measure of wave propagation since it is parallel to the local group velocity vector. Provided WKB theory 
holds, the group velocity is parallel to local wave energy flux vector. The EP flux is

$$
\vec{F}=-\overline{u_{g} v_{g}} \hat{j}+\frac{f_{0}}{N^{2}} \overline{v_{g} b} \hat{k}
$$

where $\hat{j}$ and $\hat{k}$ are, respectively, unit vectors in the $y$ and $z$ directions. The overbar is a x-average over a wavelength.

Streamline spacing in Fig. 9 is unrelated to flux magnitude; the arrows show flux direction and magnitude. Although the streamlines intersect the vertical walls $3500 \mathrm{~km}$ apart, there is no energy flux across them. Upward energy fluxes occur throughout the troposphere. Near mid-channel above $3 \mathrm{~km}$, the flux vector is just about vertical. This implies, from (3.7), the dominance of the heat transport over the momentum transport contribution. The stationary wave must tilt westward with height. Because the regions of warm air advection ahead of surface troughs are cloudy, radiative cooling will decrease northward wave heat transport thereby making the EP vectors slightly less vertical than without cloud especially in the lower troposphere. Stratospheric EP fluxes are very small in Fig. 9 and mainly horizontal thus indicating wave evanescence.

Sensitivity of stationary zonal wavenumber five to $\Phi_{C}$ is examined in Fig. 10. Without cloud, forcing is due to land-sea contrast; the resulting mid-channel amplitude of $\mathrm{v}_{\mathrm{g}}$ at the lower boundary is, as shown, $14.7 \mathrm{~m} \mathrm{~s}^{-1}$. The phase without cloud follows from (2.3). Provided dissipation is small and wavenumber five is evanescent near $z=0$, stream function phase relative to that of the heating is, as displayed, $-90^{\circ}$. Thus cooling (warming) due to land-sea temperature contrast lies to the west (east) of surface troughs. A balance is struck 
between this heating pattern and advection by perturbation and background wind as follows:

- Meridional advection of the background thermal field (background shear is westerly) gives further cooling (warming) to the west (east) of troughs.

- Advection by the background westerlies gives warming (cooling) to the west (east) of troughs since troughs (ridges) are cold (warm).

The surface wind with cloud, according to Fig. 10, varies by as much as $3 \mathrm{~m} \mathrm{~s}^{-1}$ or $20 \%$ and surface trough or ridge locations by as much as $14^{\circ}$ longitude. The surface wave amplitude is larger with cloud to the west of surface troughs since cooling amplifies the perturbation thermal and geostrophic wind patterns.

b. Response to mean cloud cooling

There is a non-zero mean over a wavelength, $\overline{\Pi(y, z)}$, of the rectified cloud cooling pattern in Fig. 1. Heating due to land-sea contrast, however, averages to zero. The response to mean cloud cooling follows from the zonally-averaged potential vorticity equation:

$$
\frac{\partial^{2} \bar{\Psi}}{\partial y^{2}}+\frac{f_{0}^{2}}{\rho_{0}} \frac{\partial}{\partial z}\left(\frac{\rho_{0}}{N^{2}} \frac{\partial \bar{\Psi}}{\partial z}\right)+\left(\frac{1}{U^{*}} \frac{\partial Q}{\partial y}-k^{2}\right) \bar{\Psi}=-\frac{i f_{0}}{k U^{*} \rho_{0}} \frac{\partial}{\partial z}\left(\frac{\rho_{0} \bar{\Pi}}{N^{2}}\right)
$$

The stream function, $\bar{\Psi}$, gives the mean zonal wind and buoyancy according to $-\partial \bar{\Psi} / \partial y$ and $f_{0} \partial \bar{\Psi} / \partial z$ respectively. Dissipation of potential vorticity in (3.8) balances cloud radiative generation.

The boundary condition at $z=0$ follows from the zonally-averaged 
thermodynamic equation: $-d f_{0} \partial \bar{\Psi} / \partial z+\bar{\Pi}=0$. Mean cloud cooling is small enough at the upper boundary that the zonal mean buoyancy perturbation or $\partial \bar{\Psi} / \partial z$ is zero. At lateral domain boundaries, the zonally-averaged ageostrophic meridional flow must be zero. Thus the only effect left in the zonally-averaged steady $u$ momentum equation is Rayleigh friction. The lateral boundary condition is thus $\partial \bar{\Psi} / \partial y=0$.

Sequential relaxation solves (3.8). Resulting tropospheric zonal wind, buoyancy, and vertical velocity fields appear in Fig. 11; the latter follows from the zonally-averaged thermodynamic equation. Mean cloud cooling creates a negative buoyancy perturbation, Fig. $11 \mathrm{~b}$, that is in thermal wind balance with a zonal wind whose maximum speed is 2.7 $\mathrm{m} \mathrm{s}^{-1}$ at the lower boundary, Fig. 11a. The wind is westerly in the northern part of the domain and easterly to the south. In most of the channel, compressional heating in weak subsidence of up to $0.06 \mathrm{~cm} \mathrm{~s}^{-}$ 1. Fig. 11c, offsets cloud cooling. Rising motion of up to $0.09 \mathrm{~cm} \mathrm{~s}^{-1}$ occurs near the northern and southern boundaries. Since there is no cloud cooling at these boundaries, adiabatic cooling is balanced by Newtonian warming.

c. Feedbacks of mean cloud cooling

Linearization decouples the responses to wavy and mean cloud cooling since they are assumed to be of the same order of a dimensionless amplitude. Thus the stationary wave forced by wavy cloud cooling does not sense the mean flow, and accompanying thermal field, set up mean cloud cooling. Useful insights are gained by departing from strict linearity to find the stationary wave response to background flow alterations by mean cloud cooling.

Add the zonal flow forced by mean cooling. Fig. 11a, to the 
background flow of Fig. 5. The jet core, Fig. 12a, shifts southward and westerly vertical shear just above cloud decreases (increases) in the northern (southern) half of the channel. The result, near the northern boundary, is little vertical shear for all $z$. Substantial changes in potential vorticity gradient, $\partial Q / \partial y$, occur near $z=0$ (see Fig. 12b). A region of negative gradient materializes in the northern half of the domain. Thus mean cloud cooling has rendered the background flow baroclinically unstable (Charney and Stern, 1962). At the same time, $\partial Q / \partial y$ doubles immediately above the cloud to the south.

The difference between stationary wavenumber five amplitude obtained with modified and unmodified mean flow appears in Fig. 13. Mean cloud cooling affects the structure below $5 \mathrm{~km}$. Wave amplification (decay) occurs in the northern (southern) half of the channel. The region negative $\partial Q / \partial y$ to the north strongly influences the local wave amplitude. According to (3.5), all wavenumbers are trapped when $\partial Q / \partial y<0$ provided $U>0$. Thus wave amplification occurs above cloud top as the wave cannot propagate energy laterally or vertically away from the energy source as efficiently as with the unmodified flow. To the south, according to (3.5), the critical wavenumber, $k_{c r}$, for the transition from wave propagation to evanescence increases in response to doubling $\partial Q / \partial y$. Propagation of energy away from the energy source due to wavy cloud cooling is facilitated. The local wave amplitude thus decreases. 


\section{Discussion}

Significant progress has occurred recently in understanding the mid-latitude planetary boundary layer (PBL) when cloud is present. Most studies of the cloud-topped PBL treat synoptic scale wind, divergence, and advection as given; they focus on the ensuing PBL thermodynamics of vertical mixing under horizontally homogeneous conditions (Lilly, 1968; Schubert, 1976 and 1979; Nicholls, 1984). Because of PBL vertical mixing (of heat, momentum and water substance), mass entrainment across the capping inversion, and latent heating through drizzle formation, the PBL can in turn influence the synoptic mass, pressure and wind fields. The present model crudely attempts to close this feedback loop. Without explicit moisture and a PBL, this study focuses on the feedbacks to the synoptic scale of a prescribed pattern of low-level stratiform cloud that is linked by longitudinal phase with the in situ synoptic wind field. The hypothesized process responsible for this linkage is cloud-induced perturbations to the field of radiative heating.

It is reasonable to not account for the PBL structure if the vertical scale of the disturbances excited by cloud cooling is much larger than the boundary layer thickness. For the linear structures forced by wavy cloud cooling whose vertical scales are on the order of the troposphere depth, this is a posteriori a reasonable assumption. Infrared cooling due to stratocumulus, however, is sharply peaked near cloud top. A caveat that can be attached to the present study is that a considerably smaller e-folding depth for the decay of cloud cooling than $10 \mathrm{~km}$ should have been used. Wavy cloud cooling with a $2 \mathrm{~km}$ e-folding depth forces stationary synoptic waves whose vertical and meridional structures are almost identical with those in Fig. 8. The conclusion is that the background zonal wind field plays a dominant role in fixing 
linear wave structures. The response to mean cloud cooling with a 2 $\mathrm{km}$ e-folding depth, however, is considerably shallower and it is hard to justify ignoring the PBL structure. It is not within the scope of this study to pursue this matter any further. Suffice it to say that the rectified nature of cloud cooling associated with wavy stratocumulus patterns could have implications that go far beyond those pointed out in this study.

A second caveat is that the cloud top radiative cooling perturbation used in the present calculations was very modest (a few deg. $K \mathrm{~d}^{-1}$ ). As pointed out in the introduction, cooling near cloud top can be an order of magnitude or more larger (Nicholls, 1984). It is thus possible that the response to cloud cooling is highly nonlinear near these concentrated layers of cooling. The resulting synoptic waves would be strongly coupled to the PBL and explicit representation of the PBL should be necessary to fully account for their structure.

The main finding of this study is that the observed coupling between summertime stratocumulus and northern Pacific surface winds and pressures is plausibly driven by cloud radiative cooling perturbations. The model produces stationary structures that resemble observed patterns not only in the phase between cloud and low-level horizontal winds but in the relationship between low-level subsidence and cloud.

There are other mechanisms besides radiative cooling that influence the linkage of the cloud-topped marine PBL with synoptic disturbances. For instance, Schubert (1976) showed that synoptic divergence influences PBL depth in a steady mixed layer PBL model. Horizontal divergence, presumably, would increase turbulent sensible and latent heat flux divergences by lowering the PBL top. A feedback on the synoptic scale motion could ensue. Another mechanism could be drizzle formation. Under conditions of strong cold air advection, drizzle 
often forms in stratocumulus (Nicholls, 1987). A net latent heating of the $\mathrm{PBL}$ follows while, at the same time, the $\mathrm{PBL}$ is stabilized through heating in the cloud layer and evaporative cooling below. Sea surface temperatures also undoubtedly influence observed stratocumulus patterns.

It is unlikely, however, that these factors, collectively or individually, can account for the observed coupling over the summertime northern Pacific. Cloud radiative cooling must be accounted for in any comprehensive theory that explains the observed linkages. 
Acknowledgments

This research was supported by NASA Contract NAS8-36150 and Office of Naval Research Contract N00014-86-K-0688. I have profited from discussions with Hampton N. Shirer of Penn State University and from suggestions by an anonymous reviewer. 


\section{APPENDIX}

The material vertical velocity $\left(w^{*}\right)$ must be zero on a horizontal solid boundary. The vertical velocity $(w)$ in the log pressure coordinate system is

$$
w=-\frac{H}{p} \omega,
$$

where $\omega=\mathrm{dp} / \mathrm{dt}$. If geometric height, $z^{*}$, is the vertical coordinate, $\omega$ can be expanded as

$$
\omega=\frac{\partial p}{\partial t}+\overrightarrow{V_{g}} \cdot \vec{\nabla} p+w^{*} \frac{\partial p}{\partial z^{*}}
$$

where, since the horizontal wind is geostrophic, the middle term on the right side drops out. At the lower boundary $w^{*}$ is zero. Thus, from (A1) and (A2),

$$
w=-\frac{H}{p} \frac{\partial p}{\partial t} .
$$

In linear theory (A3) applies at $z=0$ as well as at $z^{*}=0$. The quasigeostrophic stream function $\psi$ relates to pressure by $\psi=p /\left(f_{O} \rho_{O}\right)$; thus (A3) becomes

$$
w=-\frac{H}{\psi} \frac{\partial \psi}{\partial t}=-\frac{H}{\psi_{0}} \frac{\partial \psi}{\partial t} .
$$


The last equality follows after linearization and defining $\psi_{0}$ as a background streamfunction $\left(=p_{0} / p_{0} f_{0}\right)$. After the scale height $(H)$ is introduced, then at the lower boundary, where $w^{*}$ is zero,

$$
w=-\frac{f_{0}}{g} \frac{\partial \psi}{\partial t} .
$$

Thus, for example, the zonally-averaged thermodynamic equation at the lower boundary is

$$
\frac{\partial \bar{B}}{\partial t}-\frac{f_{0} N^{2}}{g} \frac{\partial \bar{\psi}}{\partial t}=\bar{H} .
$$




\section{REFERENCES}

Alpert, J.C., M.A. Geller and S.K. Avery, 1983: The response of stationary planetary waves to tropospheric forcing. J.Atmes.Sci., 40, 24672483.

Ashe, S., 1979: A nonlinear model of the time-averaged axially asymmetric flow induced by topography and diabatic heating. J.Atmos.Sci.36, 109-126.

Bretherton, F.P., 1966: Critical layer instability in baroclinic flows. Quart.J.Roy.Meteor.Soc. 92, 325-334.

Campana, K., 1988: Sensitivity of NMC's Medium Range Forecast Model to Changes in Cloud Climatology. Eighth Conference on Numerical Weather Prediction, Amer.Meteor.Soc., 246-251.

Charney, J.G. and M.E. Stern, 1962: On the stability of internal baroclinic jets in a rotating atmosphere. JAtmos.Sci., 19, 159-172.

Derome, J. and A. Wiin-Nielsen, 1971: The response of a middle-latitude model atmosphere to forcing by topography and stationary heat sources. Mon.Wea.Rev. 99, 351-364.

Donner, L.J. and H.-L. Kuo, 1984: Radiative forcing of stationary planetary waves. J.Atmos.Sci. 41, 2849-2868.

Edmon, Jr. H.J., B.J. Hoskins, and M.E. Mclntyre, 1980: Eliassen-Palm cross sections for the troposphere. L.Atmos.Sci.. 37, 2600-2616. 
Geisler, J.E. and R.E. Dickinson, 1975: External Rossby modes on a $\beta$ plane with realistic vertical wind shear. J.Atmos.Sci.._32, 2082 2093.

Held, I.M., 1983: Stationary and quasi-stationary eddies in the extratropical troposphere: Theory. Large-scale dynamical processes in the atmosphere, B.J. Hoskins and R.P. Pearce, Eds., Academic Press, $127-168$.

Hoskins, B.J. and D.J. Karoly, 1981: The steady linear response of a spherical atmosphere to thermal and orographic forcing. J.Atmos.Sci. 38, 1179-1196.

James I.N. and B.J. Hoskins, 1985: Some comparisons of atmospheric internal and boundary instability. J.Atmos.Sci. 42, 2142-2155.

Karoly, D. and B.J. Hoskins, 1982: The three-dimensional propagation of planetary waves. J.Meteor.Soc.Japan, 60, 109-123.

Lilly, D.K., 1986: Models of cloud-topped mixed layers under a strong inversion. Quart.J.Rov.Meteor.Soc..94, 292-309.

Lindzen, R.S., 1968: Rossby waves with negative equivalent depths Comments on a note by G.A. Corby. Quart.J.Roy.Meteor.Soc. 94, 402407.

, 1990: Some coolness concerning global warming. Bull.Amer. Meteor. Soc. 71. 288-299.

, T. Aso and D. Jacqmin, 1982: Linearized calculations of stationary waves in the atmosphere. J.Meteor.Soc.Japan, 60, 66-77. 
Nicholls, S., 1984: The dynamics of stratocumulus: aircraft observations and comparison with a mixed layer model. Quart.J.Roy.Meteor.Soc. 110, 783-820.

1987: A model of drizzle growth in warm, turbulent, stratiform clouds. Quart.J.Roy.Meteor.Soc..113, 1141-1170.

Prinn, R.G., 1977: On the radiative damping of atmospheric waves. J.Atmos.Sci. 34, 1386-1401.

Ramanathan, V., B.R. Barkstrom and E.F. Harrison, 1989: Climate and the earth's radiation budget. Phys. Today, 42, 22-32.

Randall, D.A., J.A. Coakely, Jr., C.W. Fairall, R.A. Kropfli, and D.H. Lenschow, 1984: Outlook for research on subtropical marine stratiform clouds. Bull. Amer. Meteor. Soc. 65, 1290-1301.

Rossow, W.B. and R.A. Schiffer, 1991: ISCCP cloud data products. Bull. Amer. Meteor. Soc. 72 . 2-20.

Schubert, W.H., 1976: Experiments with Lilly's cloud-topped mixed layer model. J.Atmos.Sci... 33, 436-446.

, J.S. Wakefield, E.J. Steiner and S.K. Cox, 1979: Marine stratocumulus convection. Part I: Governing equations and horizontally homogeneous solutions. J.Atmos.Sci..36, 1286-1307.

Sohn, B.-J. and E.A. Smith, 1992: The significance of cloud-radiative forcing to the general circulation on climate time scales - A satellite interpretation. J.Atmos.Sci.49, 845-860.

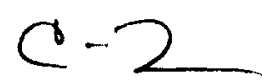


Smagorinsky, J., 1953: The dynamical influence of large-scale heat sources and sinks on the quasi-stationary mean motions of the atmosphere. Quart.J.Roy.Meteor.Soc. 79, 342-366.

Warren, S.G., C.J. Hahn, J. London, R.M. Chervin, and R.L. Jenne, 1988: Global distribution of total cloud cover and cloud type amounts over the ocean. U.S. Dept of Energy Doc. DOE/ER-0406 and NCAR Doc. NCAR/TN-317+STR. 
FIGURE CAPTIONS

Fig. 1 Schematic of wave structure showing location of cloud cooling.

Fig. 2 Phase (degrees), (a), and amplitude (dimensionless) in units of $10^{6},(b)$, as functions of background wind $(U)$ and zonal wavenumber of stream function at $z=0$ in analytical model.

Dissipation time is 10 days; phase speed is zero. $N=0.01 \mathrm{~s}^{-1}$. Shaded region in (b) is where amplitude is less than 16.

Fig. 3 Real and imaginary parts of vertical wavenumber in units of $10^{-6} \mathrm{~m}^{-1}$ for stationary waves of zonal wavenumber five in analytical model. $\mathrm{N}=0.01 \mathrm{~s}^{-1}$.

Fig. 4 Phase of factor $1-R$ (defined in text) at $z=1 \mathrm{~km}$ for zonal wavenumber five, $T_{d}=10 \mathrm{~d}$ and $U=10 \mathrm{~m} \mathrm{~s}^{-1}$. Meridional wavelength is $7000 \mathrm{~km}$ or $2 \mathrm{~W}$, where $W$ is channel width. Phase is labelled in degrees. Shaded region is where phase is less than $-90^{\circ}$.

Fig. 5 (a) Background wind, $U(y, z)$; interval $2.5 \mathrm{~m} \mathrm{~s}^{-1}$. (b) Potential vorticity gradient, $\partial \mathrm{Q} / \partial \mathrm{y}$; interval $10^{-11} \mathrm{~m}^{-1} \mathrm{~s}^{-1}$.

Fig. 6 Critical zonal wavenumber separating vertically propagating from evanescent solutions at mid-channel. Labels $\lambda_{y}=W(2 W)$ refer to wavelength in $y$ direction where $W$ is channel width.

Fig. 7 Amplitude $\left(\mathrm{s}^{-1}\right)$, and phase, of $1 /(\mathrm{imU}-\partial \mathrm{U} / \partial \mathrm{z})$ at lower 
boundary in mid-channel versus wavenumber.

Fig. 8 Amplitude of perturbation stream function (normalized as maximum value is unity) for zonal wavenumber is five.

(a) $\Phi_{C}=-90^{\circ}$; (b) $\Phi_{C}=90^{\circ}$.

Fig. 9 Eliassen-Palm flux for wavenumber five and $\Phi_{C}=-90^{\circ}$.

Fig. 10 Perturbation geostrophic wind at lower boundary at midchannel, $v_{g}$, and phase of stream function, $\lambda$, at lower boundary as functions of the phase of stratocumulus-induced heating field. If heating due to land-sea contrast goes as $\cos (k x)$, stream function is $\cos (k x+\lambda)$. Also shown are $v_{g}$ and $\lambda$ if clouds do not occur and the wave is driven by fixed surface heating only.

Fig. 11 Tropospheric response to mean cloud cooling (a) Zonal flow forced by mean stratocumulus cooling. Contour interval is 0.6 $\mathrm{m} \mathrm{s}^{-1}$. (b) Buoyancy perturbation; interval $0.03 \mathrm{~m} \mathrm{~s}^{-2}$. (c) Vertical velocity; interval $0.03 \mathrm{~cm} \mathrm{~s}^{-1}$. Dashed (solid) contours indicate negative (positive) values. Zero contours are labeled.

Fig. 12 (a) Background wind supplemented by contribution due to mean cloud cooling; interval $2.5 \mathrm{~m} \mathrm{~s}^{-1}$. (b) Potential vorticity gradient (negative region shaded) corresponding to flow in (a); interval $10^{-11} \mathrm{~m}^{-1} \mathrm{~s}^{-1}$.

Fig. 13 Change in stream function amplitude when mean flow forced by cloud cooling is added to background flow; wavenumber five. $\Phi_{C}=90^{\circ}$. Values are normalized to unity. 


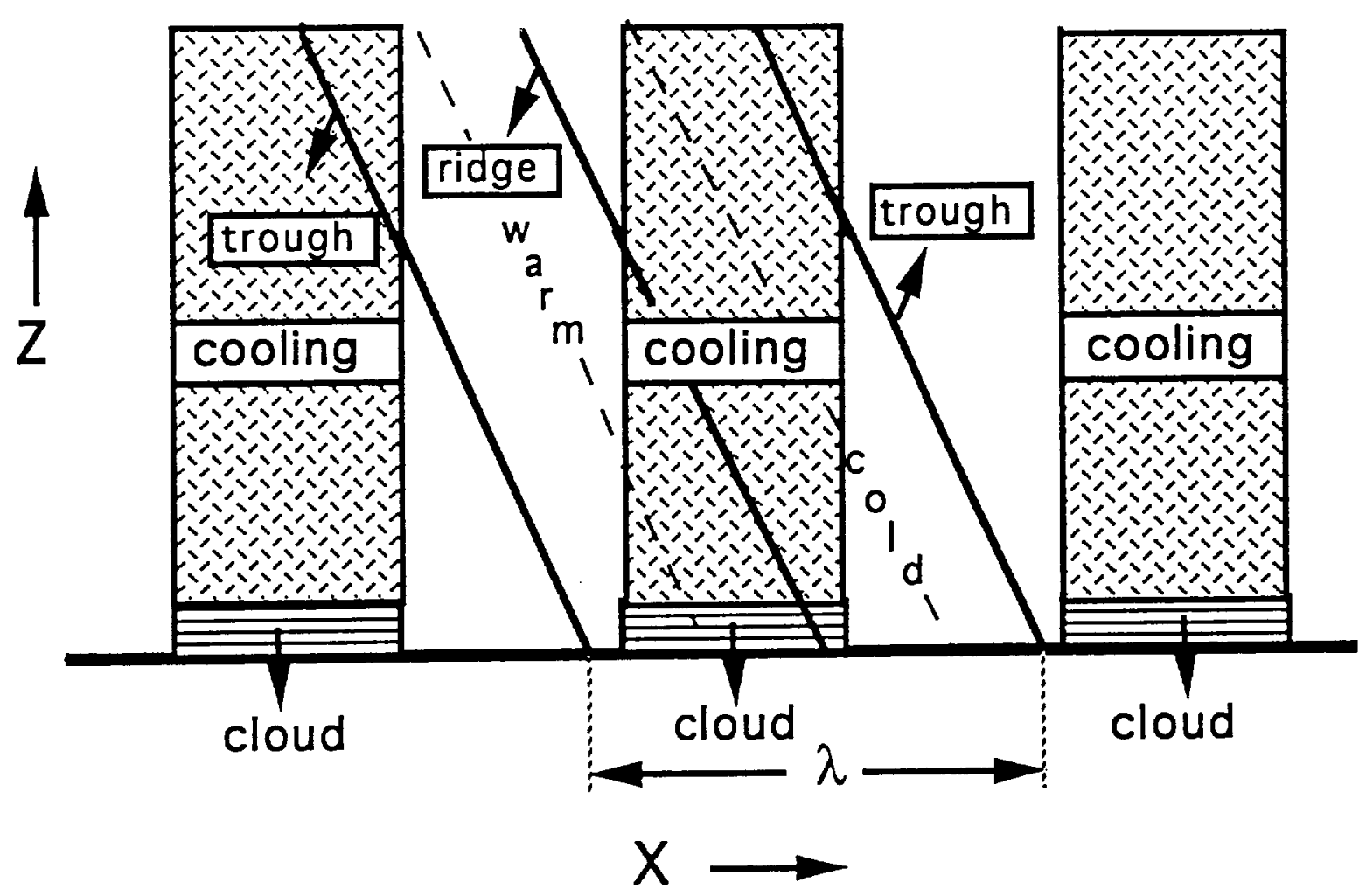

Fig. 1 


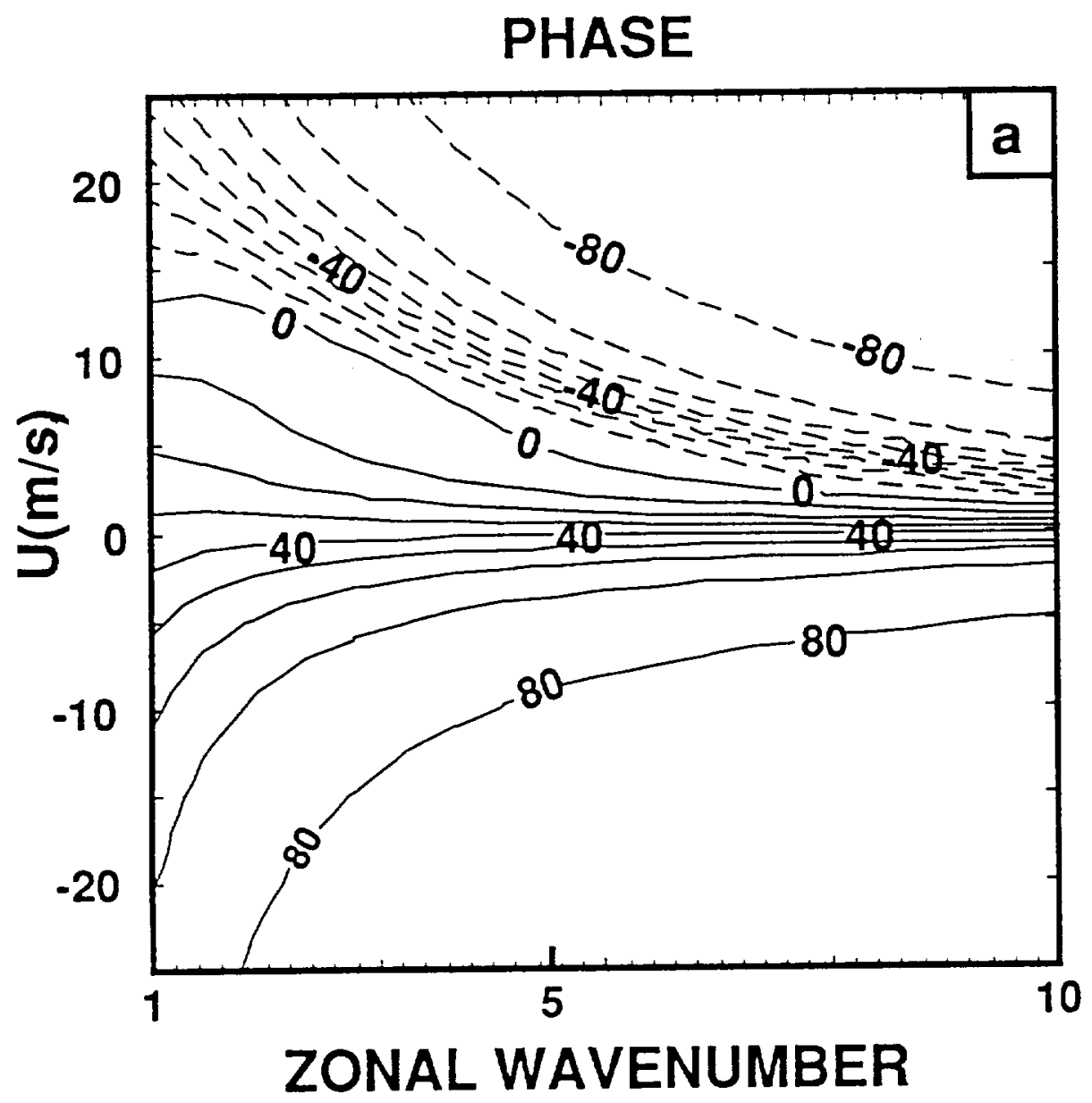

Fig. $2 a$ 


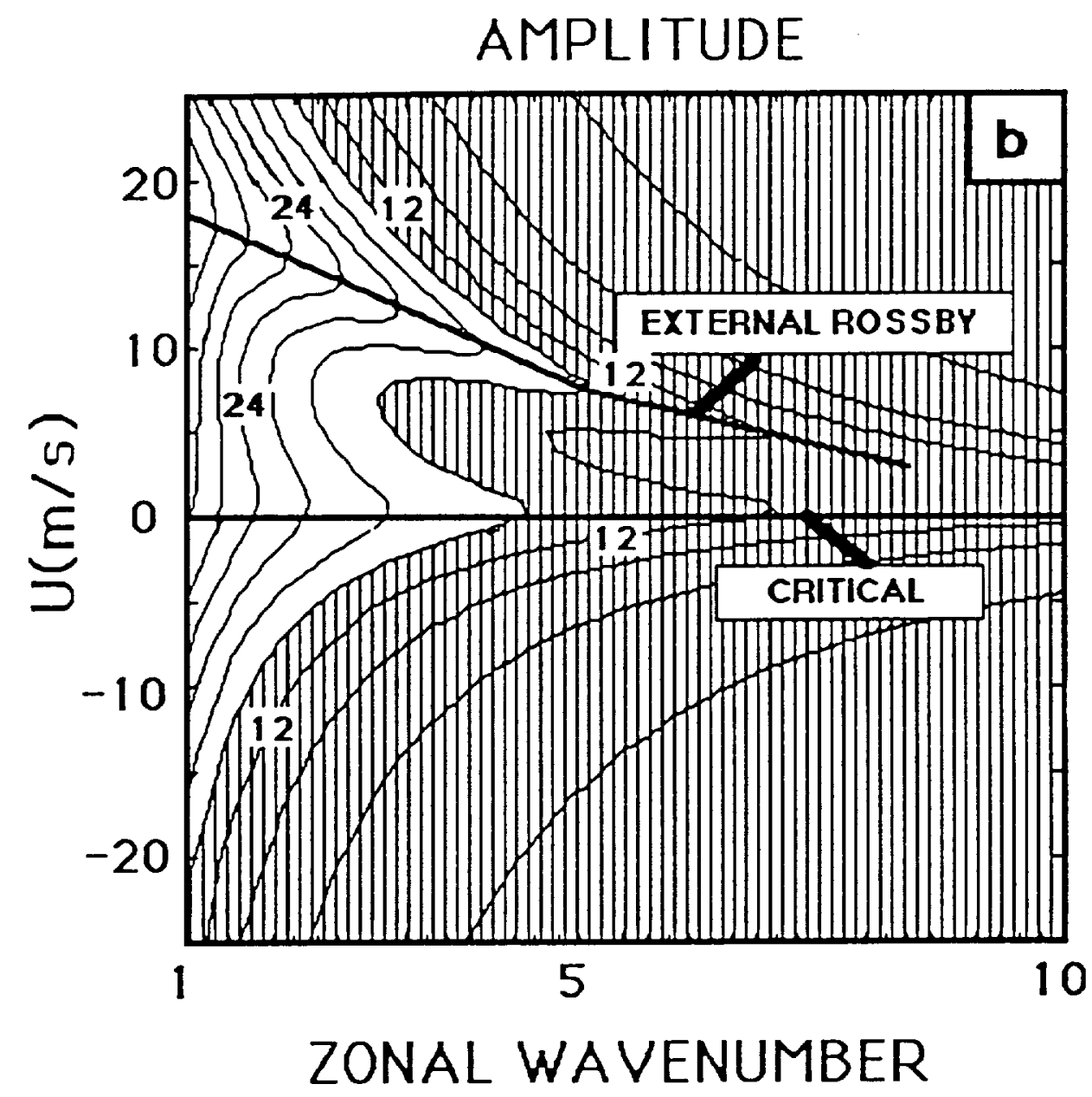

Fig. $2 b$ 


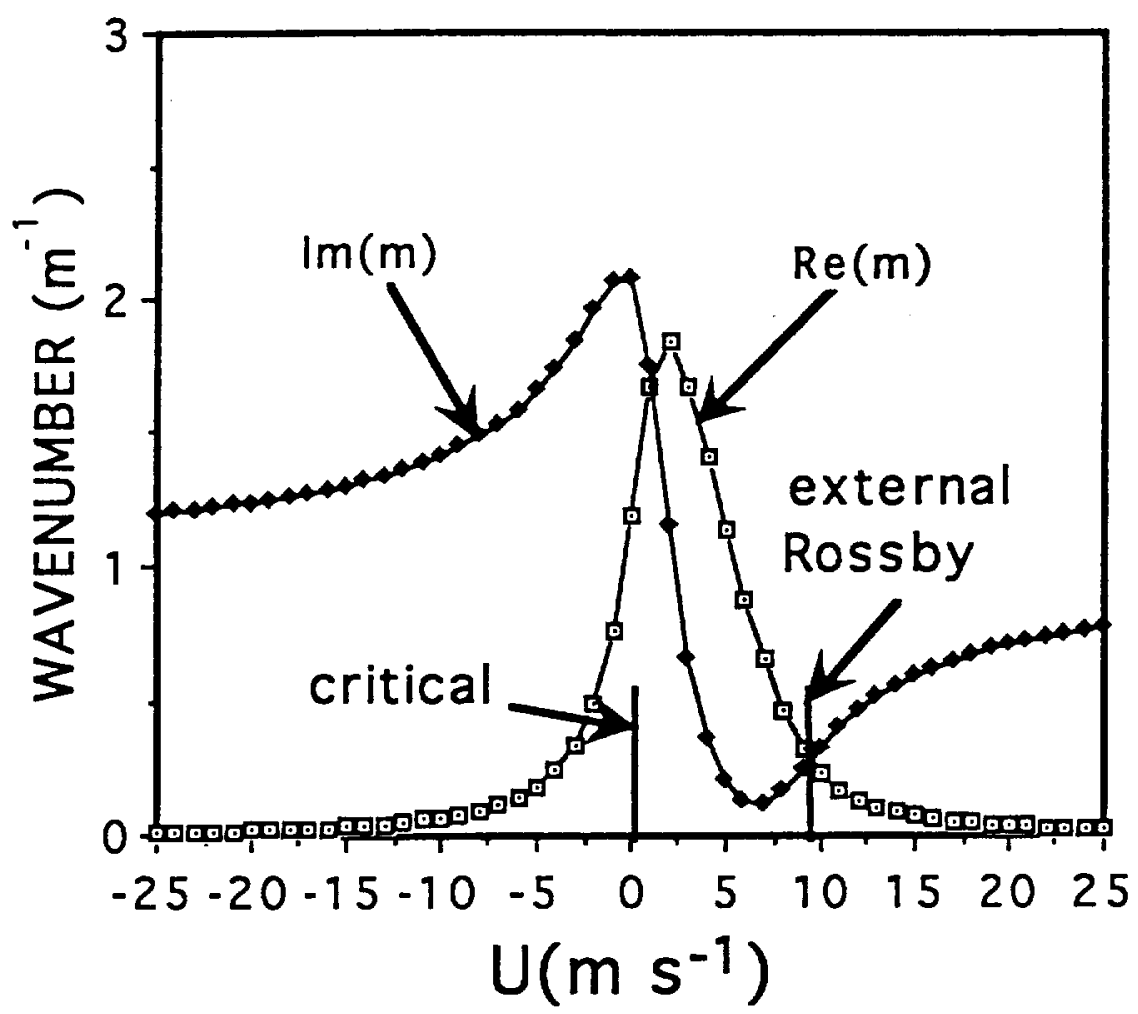

Fig. 3 


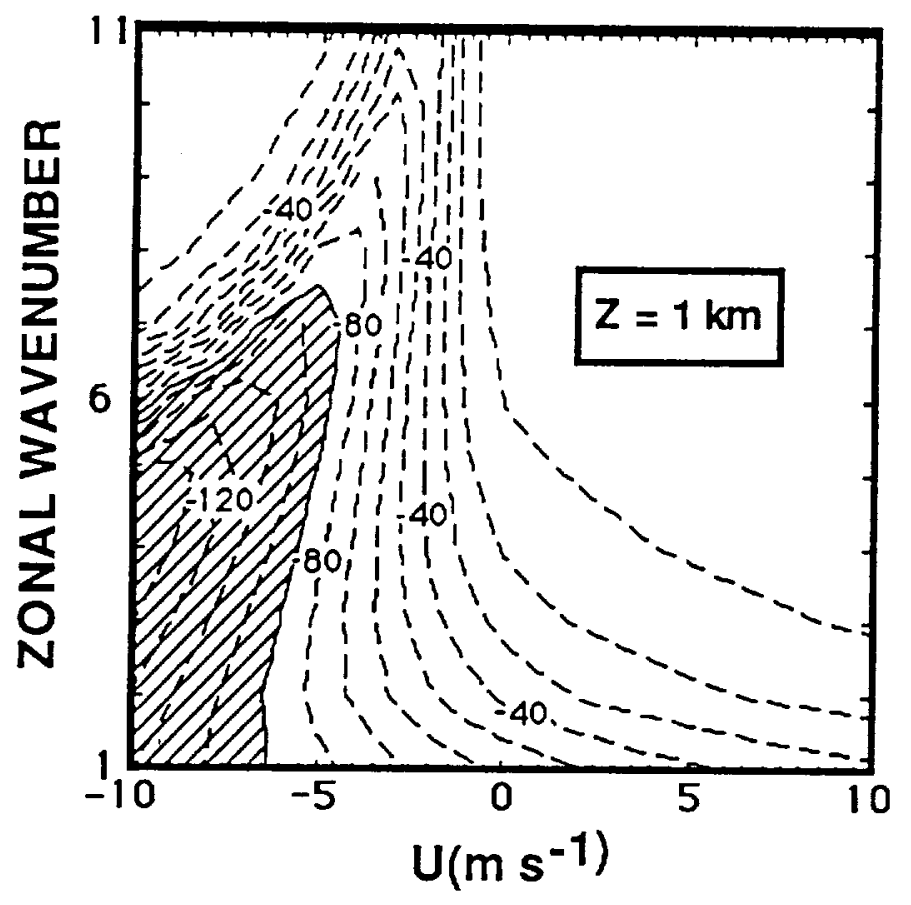

Fig. 4 

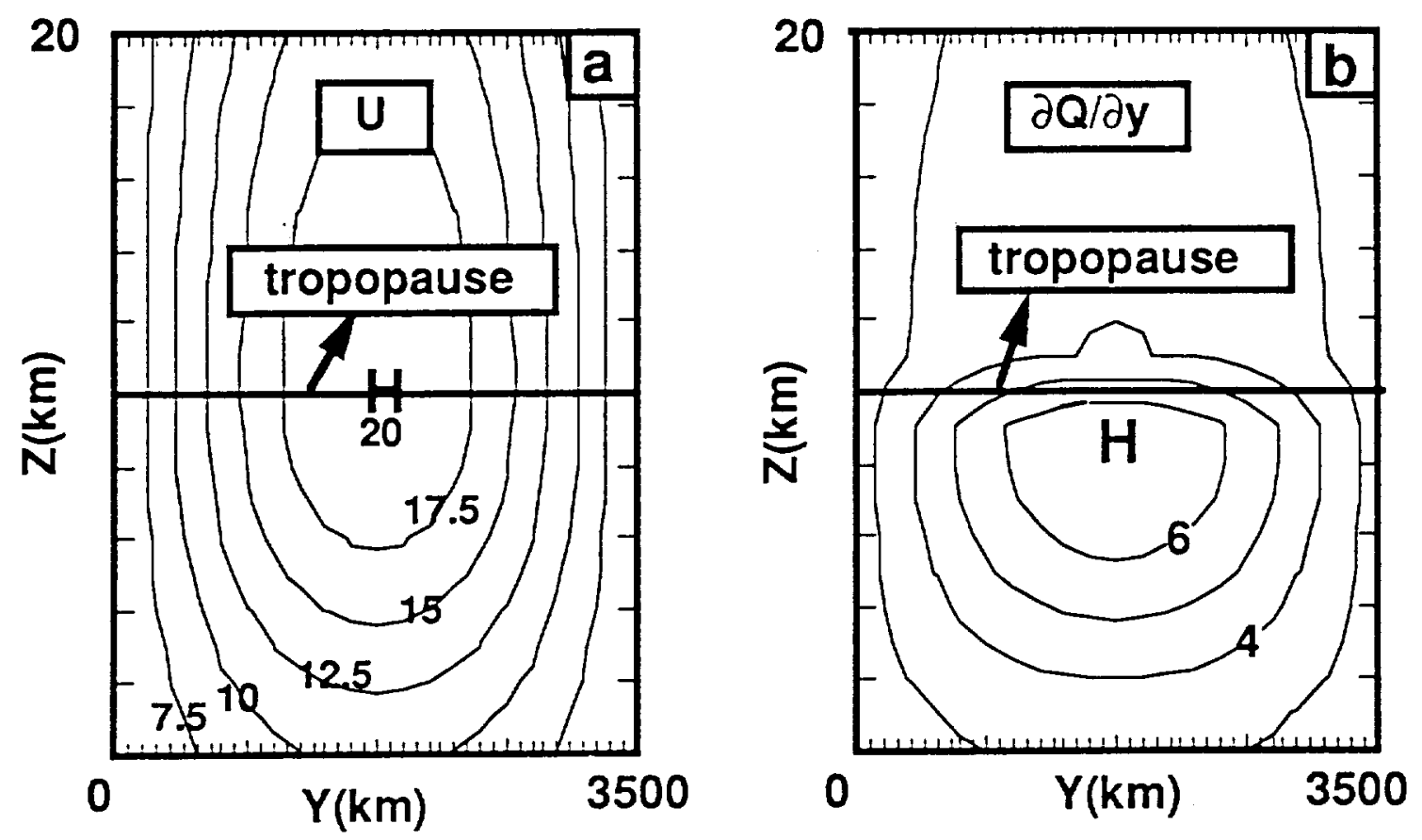

Fig. 5 


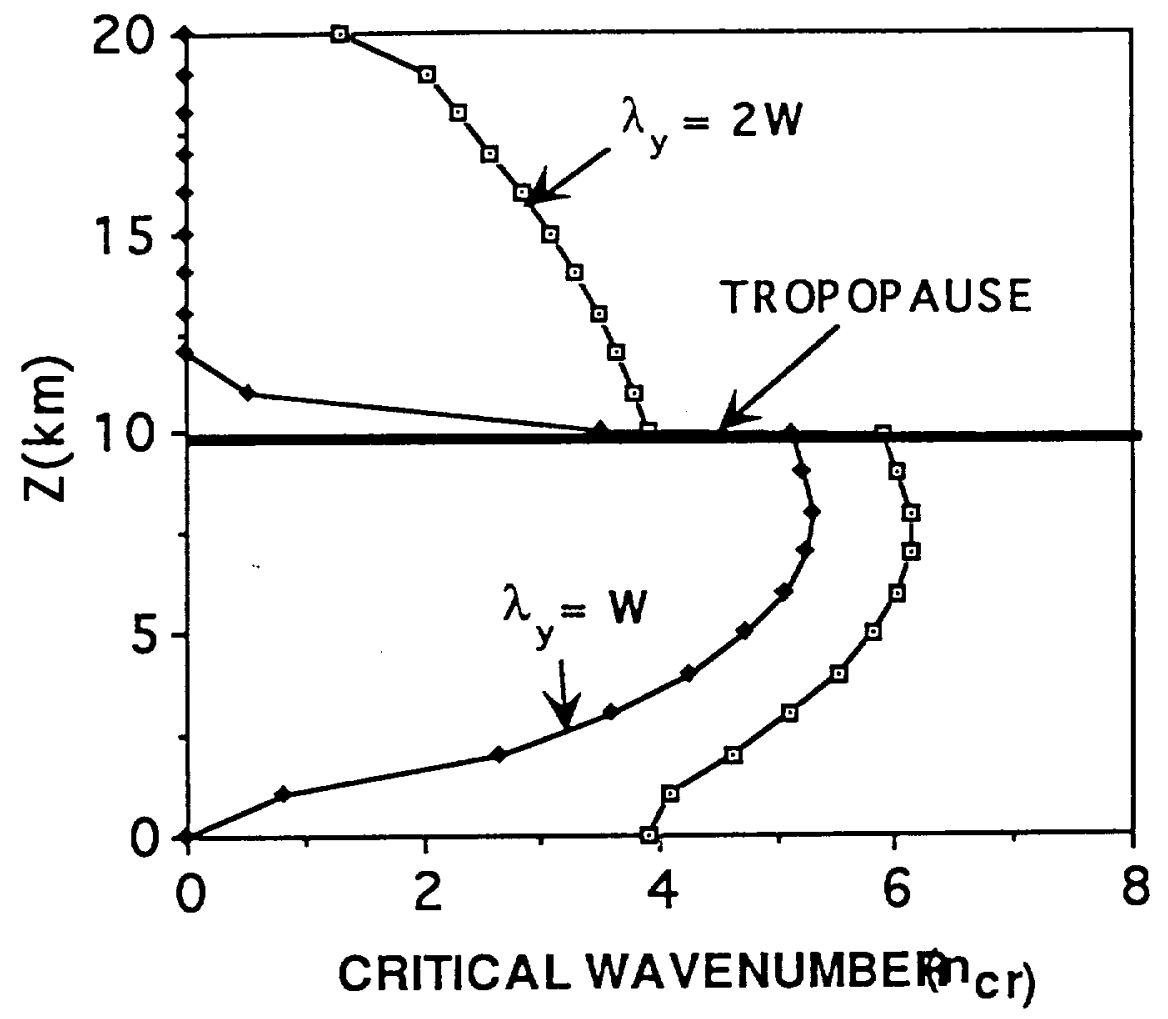

Fig. 6 


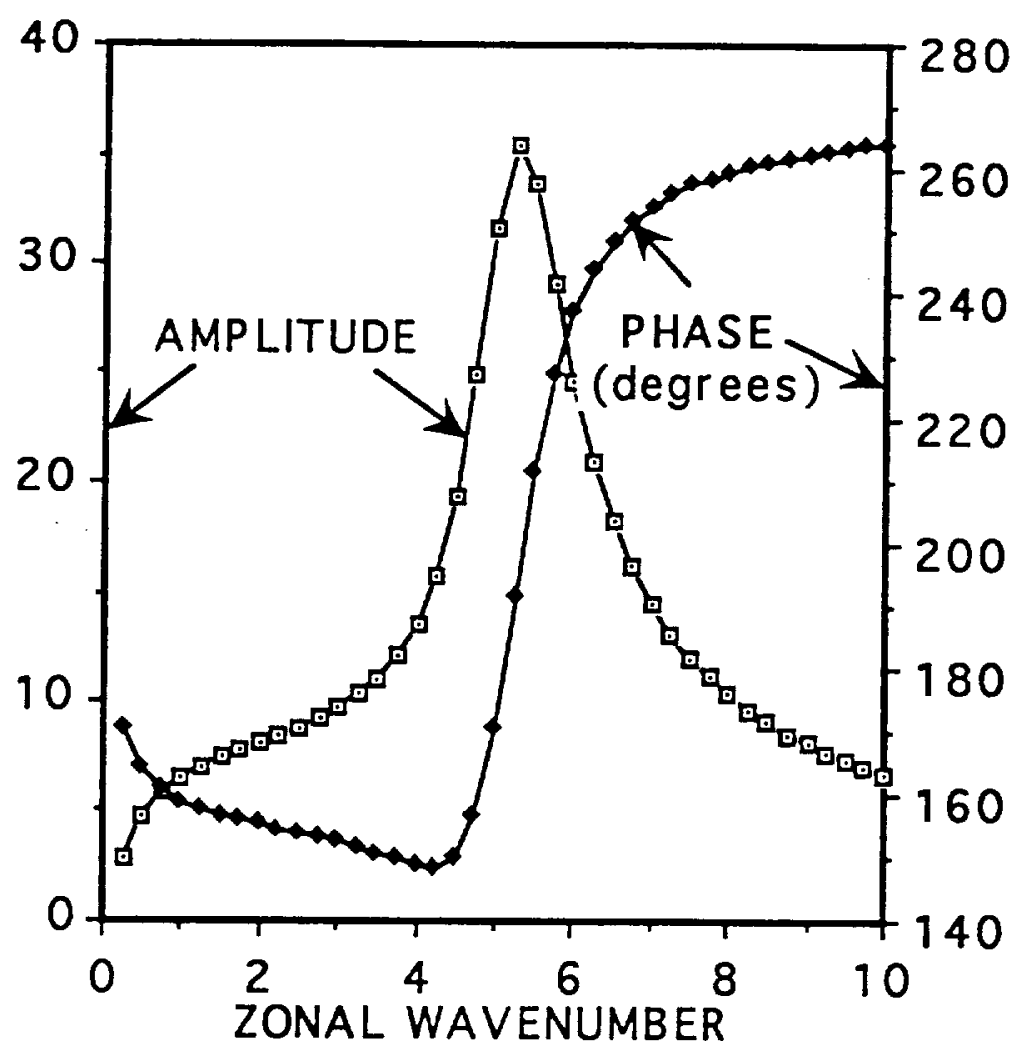

Fig. 7 

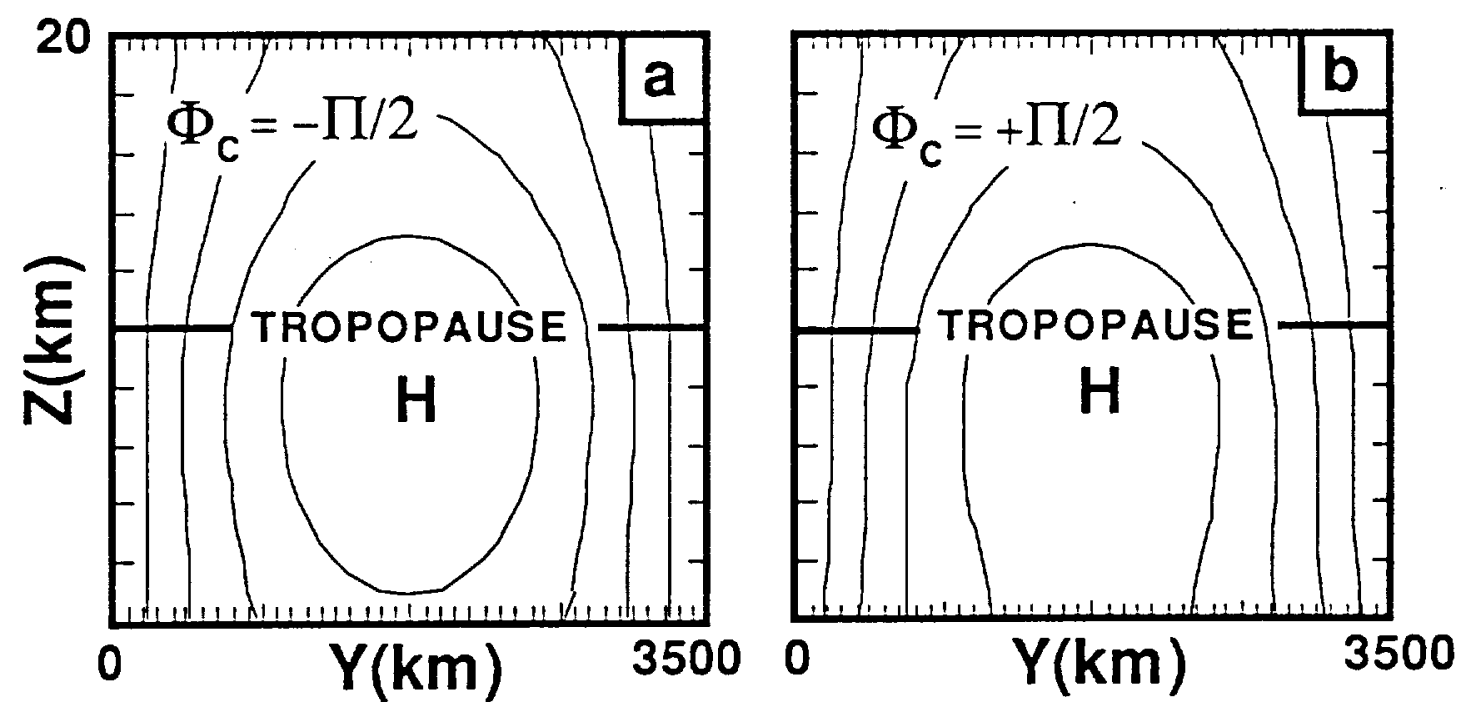

Fig. 8 


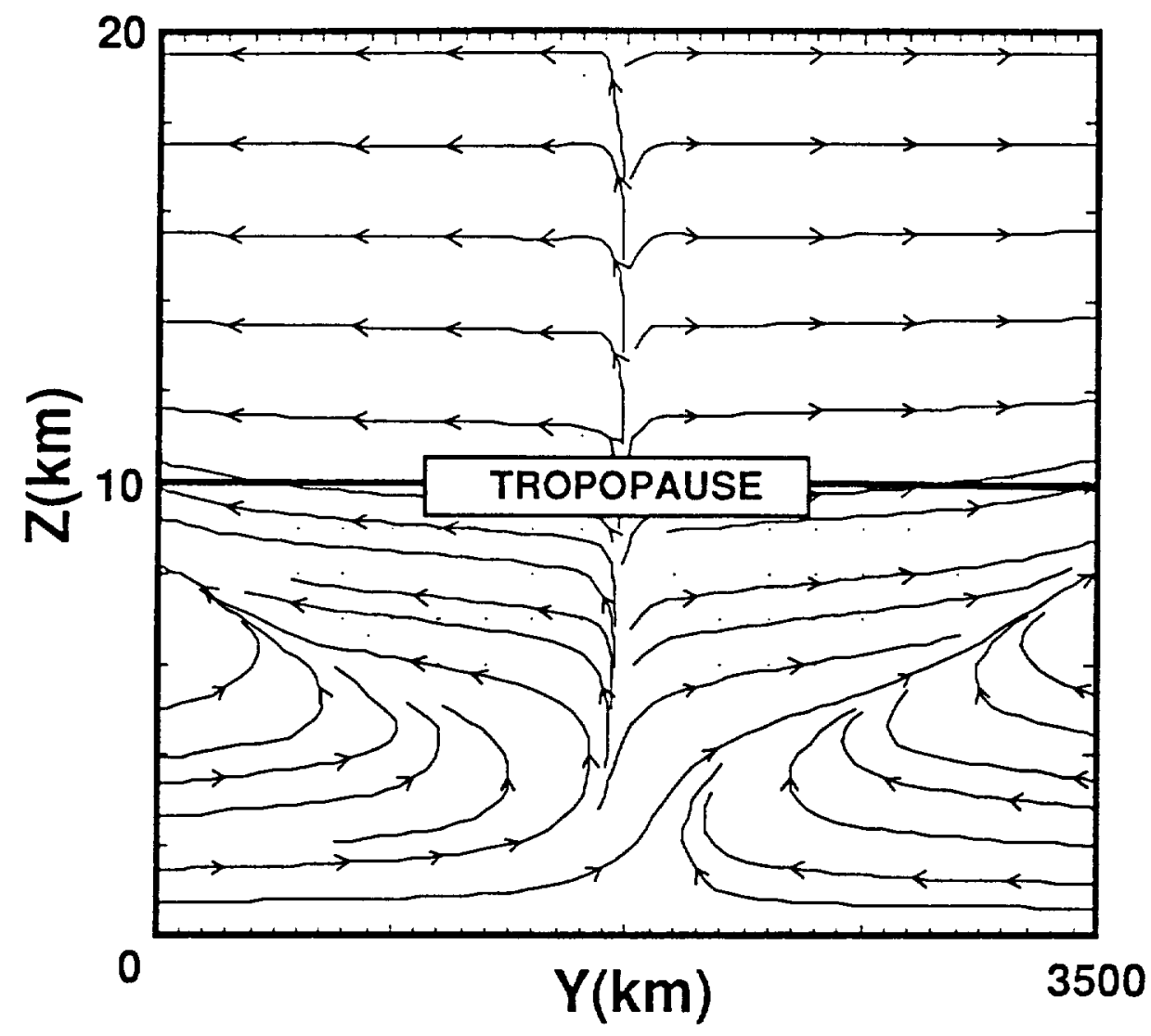

Fig. 9 


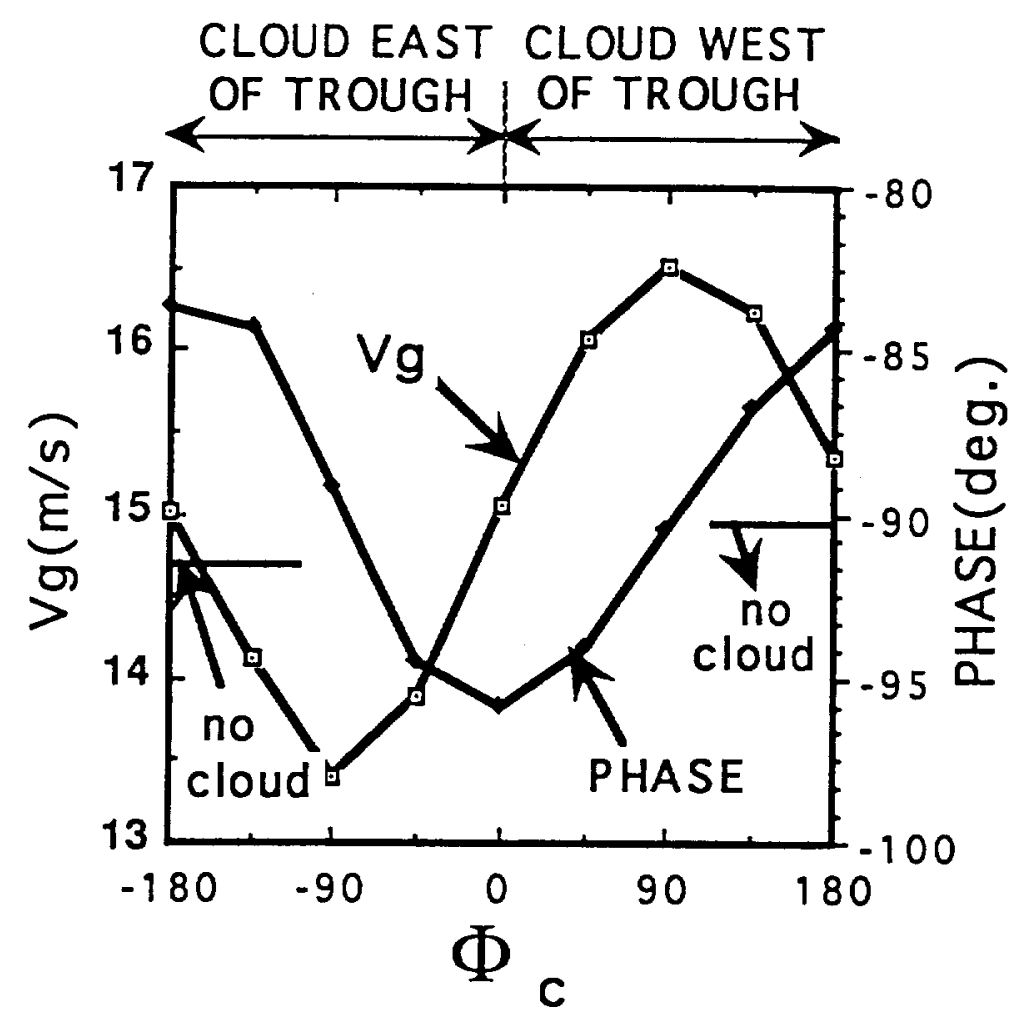

Fig. 10 


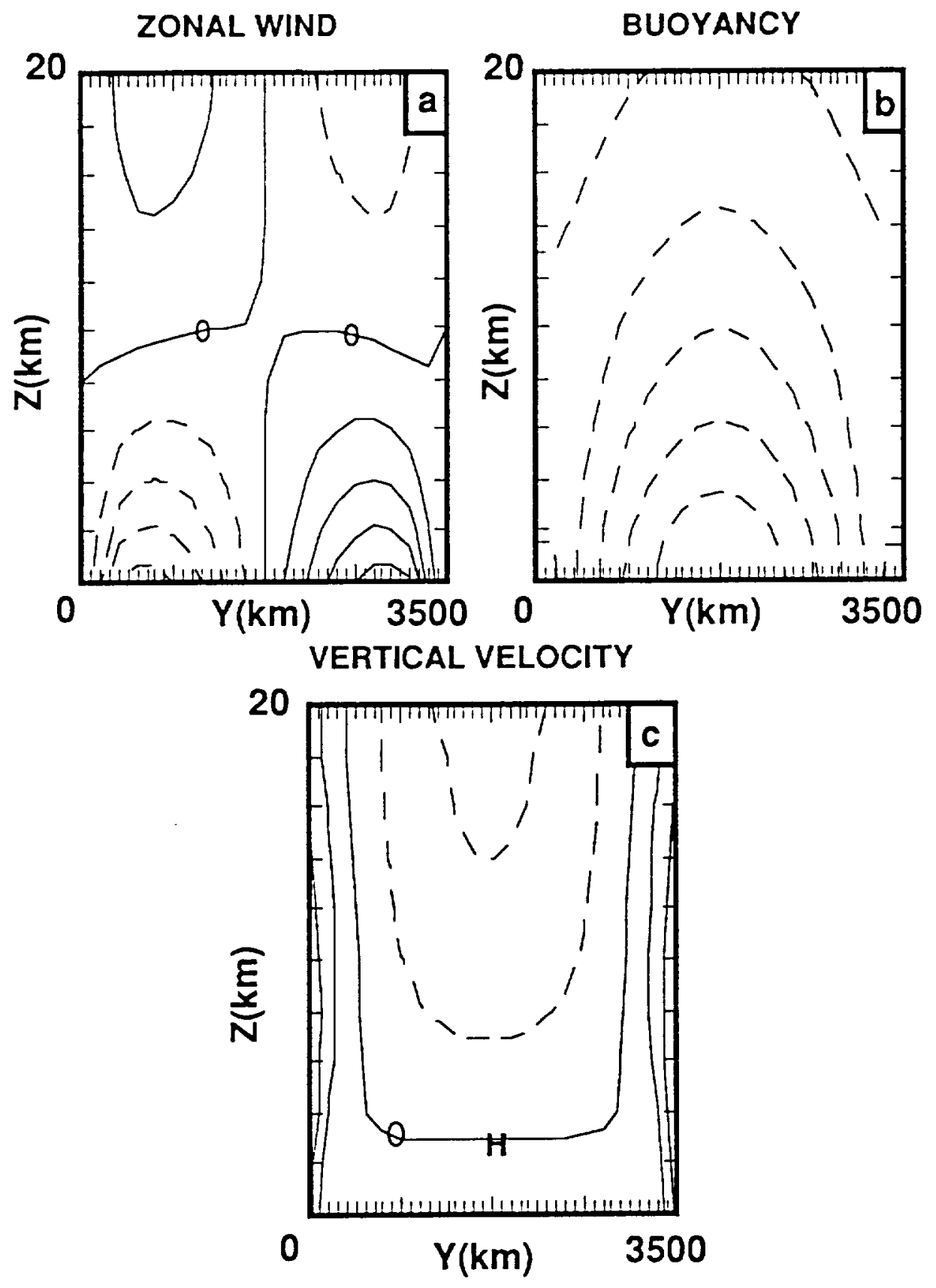

Fig. 11 


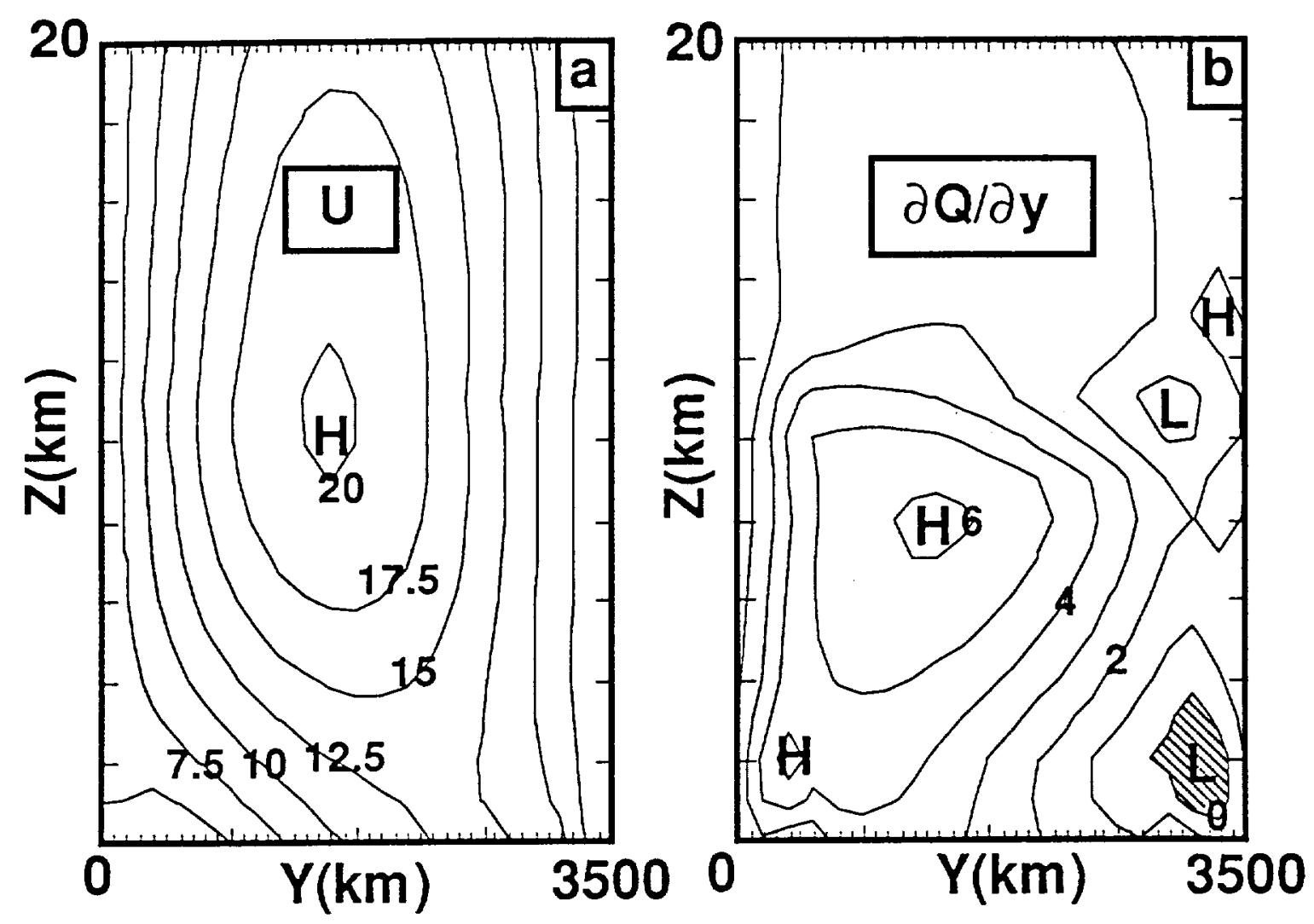

Fig. 12 


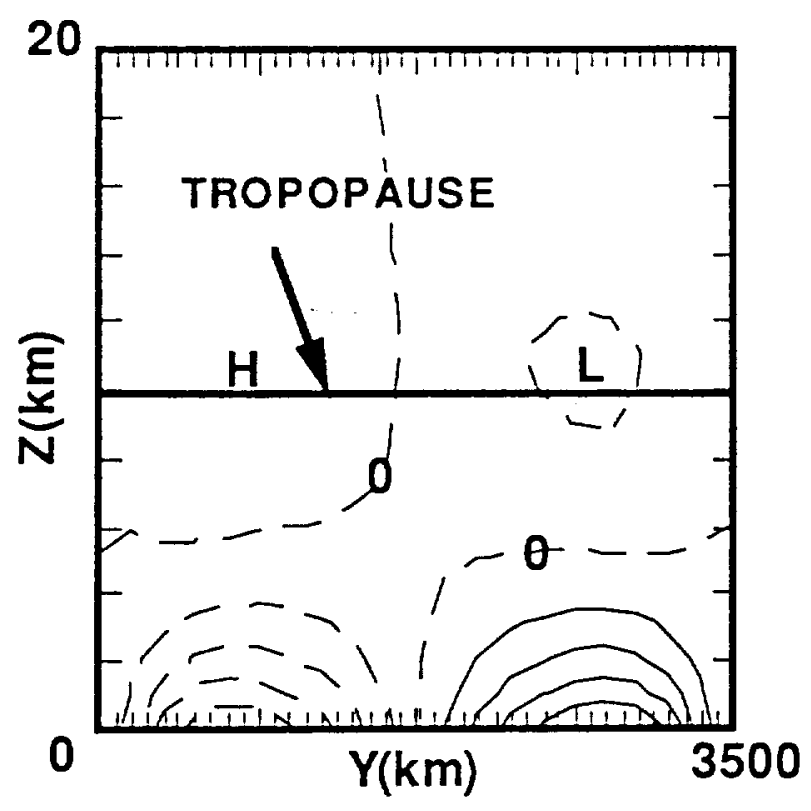

Fig. 13 



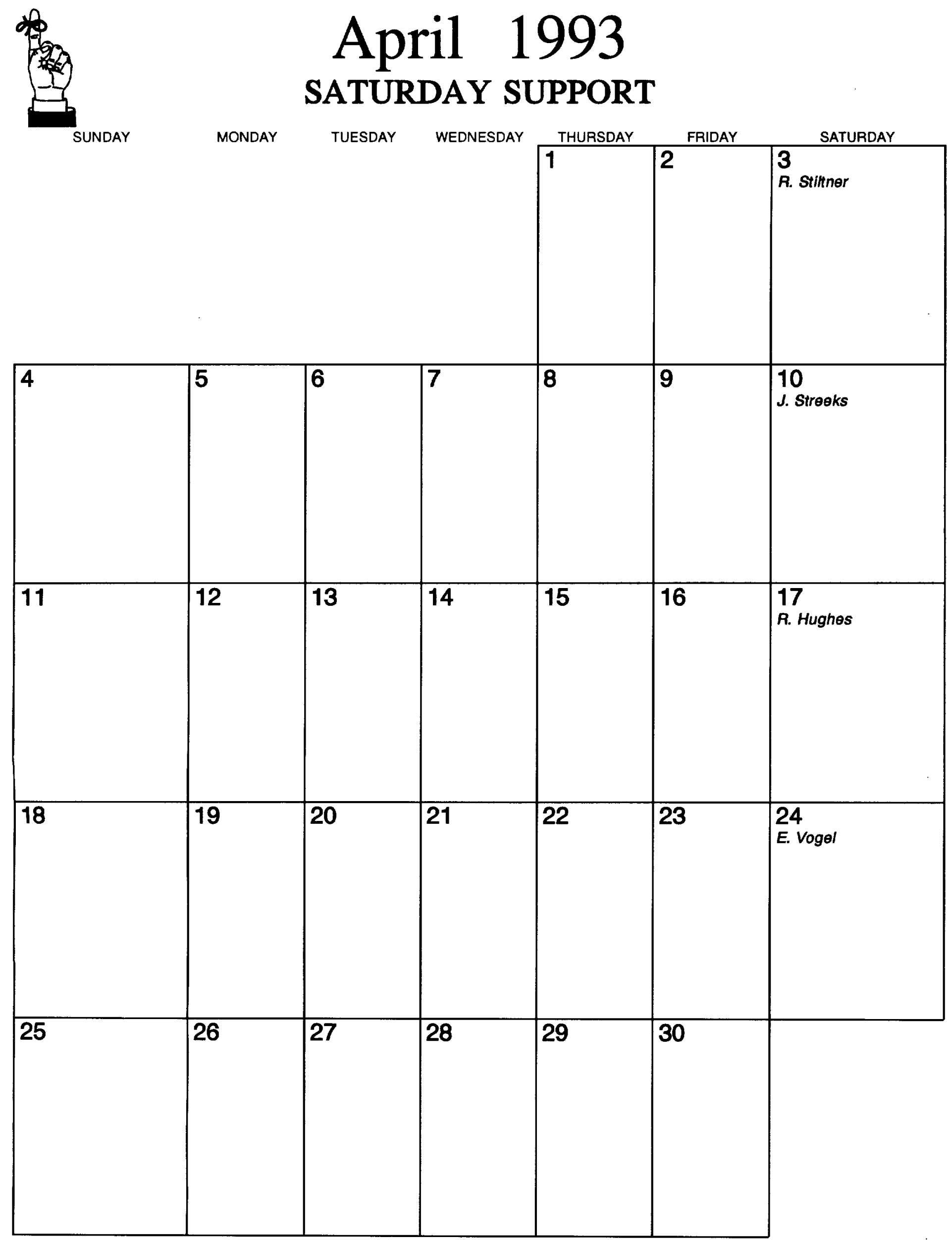

